\title{
On the 27-Plet Unitary Symmetry Operator
}

\author{
J. A. Castilho Alcaras* and L. C. Biedenharn \\ Department of Physics, Duke University, Durham, North Carolina $27706^{\dagger}$ \\ AND \\ K. T. HeCht AND G. NeELY ${ }^{\ddagger}$ \\ Department of Physics, University of Michigan, Ann Arbor, Michigan $48104^{\S}$
}

Received January 7, 1970

\begin{abstract}
The unitary spin-tensor operator transforming as the representation [4 2 0] in $S U(3)$ (the 27-plet) is discussed in detail. Using a recent result for the unique (nonarbitrary) splitting of the multiplicity, complete algebraic formulas are determined for the complete 27-plet operator. Symmetry properties of the 27-plet operator are given. Applications to both particle and nuclear physics are discussed.
\end{abstract}

\section{INTRODUCTION}

The 27-plet unitary spin operator-the $S U(3)$ tensor operator transforming as

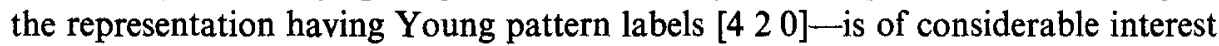
for both physical and mathematical reasons. The 27-plet operator occurs in physical problems in both (elementary) particle physics and in nuclear structure (shell-model) physics. In particle physics, the 27-plet operator occurs most prominently in symmetry-breaking applications: in second-order corrections to the Gell-MannOkubo mass formula, in weak interaction Hamiltonians, and in electromagnetic interactions. In nuclear structure physics the 27-plet operator is of interest in the $S U(3)$ (rotator) model of Elliot which singles out a dominant $S U(3)$ multiplet (the operator then directly splits the degeneracy). In shell model calculations, using a fractional parentage approach, wherein several $S U(3)$ representations may be

* On leave from the Instituto de Fisica Teorica, Sao Paulo, Brazil under a fellowship of the Conselho Nacional de Pesquisas (CNPq)-Brazil.

${ }^{\dagger}$ Supported in part by the U.S. Army Research Office (Durham) and the National Science Foundation.

\# Present address: Physics Department, Fisk University, Nashville, Tennessee 37203.

$\S$ Supported in part by the U. S. Office of Naval Research, Contract No. Nonr-1224(59). 
mixed, the 27-plet operator occurs as one of the most important operators which leads to such mixings. (We discuss these applications in Section 4 below.)

From the point of view of mathematical physics the $\langle 420\rangle$, (27-plet), operator plays a most interesting role: It is the simplest operator in the simplest unitary group displaying a nontrivial multiplicity. To be sure, the octet operator $\langle 210\rangle$, does indeed have multiplicity two (the familiar " $F$ " and " $D$ " operators of GellMann); however, this multiplicity is completely split by an involutory operationthe "conjugation parity" [1]. In this limited sense, multiplicity two is not general. By contrast, the 27-plet operator displays a multiplicity three for its diagonal matrix elements; two of the three systems have the same conjugation parity and in consequence the general multiplicity structure now appears. The principal new result of the present paper is a complete algebraic determination of the 27-plet unitary spin operator in a basis that resolves the multiplicity structure in a unique and nonarbitrary (canonical) way.

For the evaluation of $S U(3)$ operator matrix elements the simplest procedure makes use of the "canonical scheme," that is, the subgroup chain $S U(3) \supset S U(2)$. This chain induces a complete labeling scheme $\left(I^{2}, I_{z}\right.$, and $Y$ in particle physics). For nuclear physics this is not the physically relevant scheme; rather one is forced to use the chain $S U(3) \supset R(3)$, where $R(3)$ is the physical (orbital) angular momentum of the shell model basis. This latter scheme introduces difficult technical problems which we do not attempt to discuss here. However, for application to nuclear physics, our operator evaluation leads naturally to the determination of several SU(3) recoupling (Racah) coefficients (which are subgroup chain independent). Since these coefficients are of direct application in nuclear physics we have included a short tabulation in Appendix I.

In Section 2 we discuss in detail the method of calculation of the 27-plet operator. In Section 3 we discuss several symmetry properties possessed by the 27-plet operator matrix elements, including an important new symmetry property based on asymptotic limits. In Section 4 we discuss applications to particle and nuclear physics. The tables are given in Section 5, along with several illustrative examples, so that the tabular results are directly accessible without detailed study of the method of calculation.

\section{Calculational Procedures}

Before beginning with the details of the methods of calculation, it might be useful to indicate briefly the underlying reasons why the structural problems presented by the 27-plet operator are of interest. (A more detailed, and broader, survey has been given recently by Wigner [2].) Briefly, the problem of multiplicity in groups larger than $S U(2)$ has been the major stumbling block in the construction 
of the associated Racah-Wigner calculus; the multiplicity appears, at least superficially, to introduce unavoidable arbitrariness in the construction. A canonical solution - that is, a solution involving no free choices-has, however, recently been proved to exist for the $S U(3)$ group [3]. In consequence, there exists in fact only arbitrariness associated with phase conventions in the construction of any, and hence all, operators in $S U(3)$. A systematic approach to all calculations involving unitary spin $(S U(3))$ is now feasible.

The canonical construction given in Ref. [3] is necessarily rather abstract. Hence the explicit construction of the simplest example of a nontrivial multiplicity -the 27-plet operator-is of considerable value simply to illustrate the principles involved in the general construction. There is rather more than just this involved, for this explicit example also serves to provide a sort of "testing ground" for various conjectures of a structural nature. [In Section (3), below, we give illustrations of such conjectures.]

Finally let us note that this determination of the 27-plet operator is unavoidably (algebraically) complicated. To obviate the possibility of error, we have actually carried out two (algebraically) independent evaluations: (a) construction via the product $\left[\begin{array}{lll}2 & 0 & 0\end{array}\right] \times\left[\begin{array}{lll}2 & 2 & 0\end{array}\right] \rightarrow\left[\begin{array}{lll}4 & 2 & 0\end{array}\right]$ and (b) construction via the product $\left[\begin{array}{lll}2 & 1 & 0\end{array}\right] \times\left[\begin{array}{lll}2 & 1 & 0\end{array}\right] \rightarrow\left[\begin{array}{lll}4 & 2 & 0\end{array}\right]$. Both procedures are equivalent for the canonical construction (ultimately they each involve six "quark" operators); but, since they employ different "paths" (arranging the work very differently) they provided a valuable-and very stringent-check on the correctness of the results. (Other checks are discussed in Section 3.)

Not all readers will be interested in the details to follow; for such readers the examples of the use of the tables (Section 5) and the "translation guide" for relating various notations (Appendix II), will enable the remainder of this section to be skipped.

\section{Notational Preliminaries}

Let us restrict attention solely to the group $U(3)$.

Wigner operators are defined as unit tensor operators; equally well, one may define the Wigner operators by their effect on a generic state vector, $|(m)\rangle$, of $U(3)$, where $(m)$ denotes a Gelfand pattern:

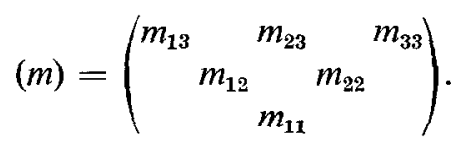

Here the $m_{i j}$ are nonnegative integers, obeying the betweenness condition $m_{i, j+1} \geqslant m_{i j} \geqslant m_{i+1, j+1}$. Every such array is associated with a unique state vector of an irrep of $U(3)$ and conversely. (The restriction to $S U(3)$ is $m_{33}=0$.) 
A Wigner operator, denoted by the array

$$
\left(\begin{array}{cccc} 
& & \mu_{11} & \\
& \mu_{12} & \mu_{22} & \\
M_{13} & & M_{23} & M_{33} \\
& M_{12} & M_{22}
\end{array}\right)
$$

carries two sorts of labels: (1) a Gelfand pattern, denoted by

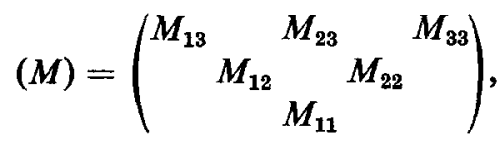

which specifies how the operator transforms under the generators, and (2) an "operator pattern" denoted by

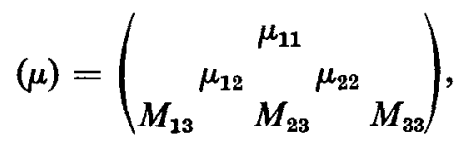

which specifies the shifts, when acting on an initial state vector $\left|(m)^{\text {intial }}\right\rangle$ :

$$
\begin{aligned}
& m_{13}^{\text {final }}-m_{13}^{\text {initial }}=\Delta m_{13}=\mu_{11} \\
& m_{23}^{\text {final }}-m_{23}^{\text {initial }}=\Delta m_{23}=\mu_{12}+\mu_{22}-\mu_{11} \\
& m_{33}^{\text {final }}-m_{33}^{\text {initial }}=\Delta m_{33}=M_{13}+M_{23}+M_{33}-\mu_{12}-\mu_{22} .
\end{aligned}
$$

Just as the Gelfand pattern distinguishes state vectors of the same weight, so the operator pattern distinguishes operators inducing the same shifts $\Delta$. (This is discussed in more detail below.)

A Wigner operator in $U(3)$ is, at the same time, a sum of Wigner operators in the subgroup $U(2)$; expressed symbolically, this statement becomes the subgroup reduction formula:

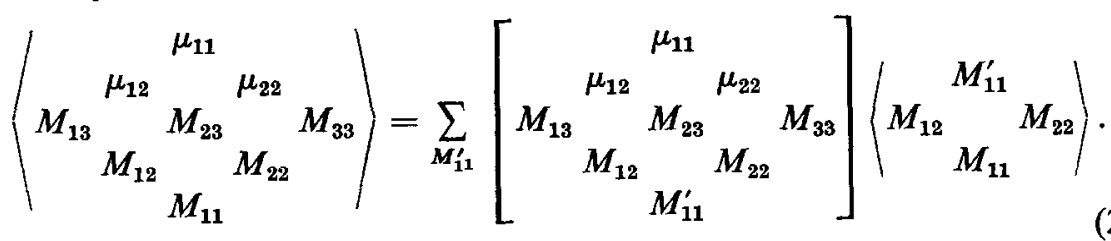

In this equation, [...] denotes a reduced Wigner operator (in particle physics this is called an "isoscalar factor" [4]). The $U(2)$ Wigner operators in Eq. (2.4) are precisely the usual ones, whose matrix elements are the well-known "ClebschGordan Coefficients."

(The U(2) Wigner operators and Wigner coefficients are given in the "pattern notation" in Appendix II.)

The great advantage of the reduction law is that it permits one to tabulate a 
simpler object, the reduced Wigner operator. Such an operator may be defined by its action on $U(3)$ state vectors, but in order that it be a proper operator, it must however act on state vectors maximal in $U(2)$, i.e., $m_{11}=m_{12}$. Thus one has

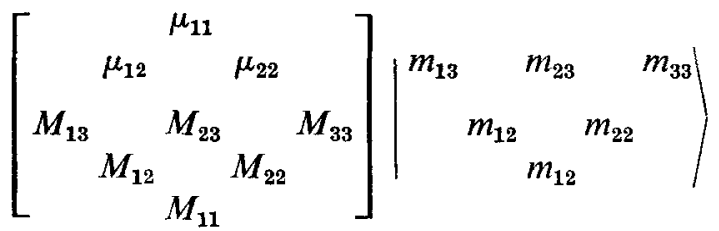

$$
\begin{aligned}
& -\#\left|\begin{array}{c}
m_{13}+\mu_{11} m_{23}+\left(\mu_{12}+\mu_{22}-\mu_{11}\right) m_{33}+\left(M_{13}+M_{23}+M_{33}-\mu_{12}-\mu_{22}\right) \\
m_{12}+M_{11} m_{22}+\left(M_{12}+M_{22}-M_{11}\right) \\
m_{12}+M_{11}
\end{array}\right|
\end{aligned}
$$

Here \# denotes an algebraic function, that is, the explicit matrix element of the reduced Wigner operator, also called "reduced Wigner coefficients," to distinguish them from the "Wigner coefficients" which are the matrix elements of the components of the full Wigner operators.

From this equation one sees that a reduced Wigner operator causes two shifts:

$$
\begin{aligned}
& \Delta_{3}=\left(\Delta m_{13}, \Delta m_{23}, \Delta m_{23}\right), \text { in } U(3) \text { labels } \\
& \Delta_{2}=\left(\Delta m_{12}=M_{11}, \Delta m_{22}=M_{12}+M_{22}-M_{11}\right) \text { in } U(2) \text { labels. }
\end{aligned}
$$

It is helpful to note that there is an analogy between the shift pattern of an

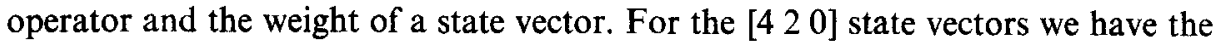
familiar 27-plet weight diagram:

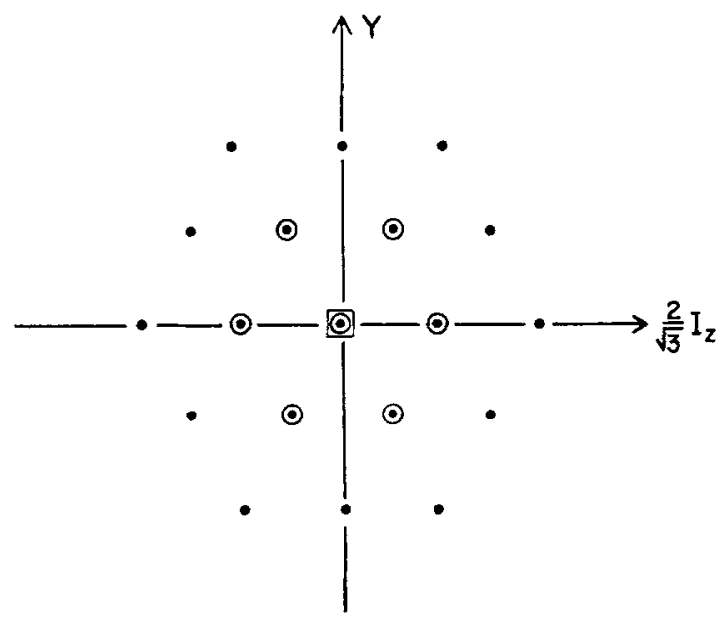

FIG. 1. Weight diagram for the irrep [420] of $S U(3)$. 
We see that there are 12 weights (the outer points) with multiplicity one; 6 with multiplicity two (the circled points) and 1 with multiplicity three (the center point).

The multiplicity of the state vectors is resolved by the Weyl branching law; the resolution is denoted by the Gelfand pattern [1]. The multiplicity of the Wigner operators is resolved-for $U(3)$ at least-by the existence of an operator branching law; the resolution is denoted by an operator pattern.

The construction of extremal reduced Wigner operators (which have multiplicity one) has an elegant solution in the "pattern calculus" of Biedenharn and Louck [5]. We can use these techniques to check some of the multiplicity one 27-plet operators given in the tables of Section 5.

The determination of the multiple operators is the most difficult task. For this purpose, we use the Factorization Lemma of Ref. [3]. Consider a boson operator in $U(3) * U(3)$, that is, a polynomial operator made up of the nine basic boson operators $a_{j}{ }^{i}$ (with $i, j$ running over $1,2,3$ ) transforming as the two $U(3)$ Gelfand patterns $(M)$ and $\left(M^{\prime}\right)$, where $M_{i 3}=M_{i 3}^{\prime}$. Briefly put, this lemma asserts that the boson operator

$$
B\left(\begin{array}{ccc} 
& \multicolumn{3}{c}{M_{11}^{\prime}} & \\
& M_{12}^{\prime} & M_{22}^{\prime} \\
M_{13} & M_{23} & M_{33} \\
& M_{12} & M_{22}
\end{array}\right)
$$

may be factorized into the product of two $U(3)$ operators, each acting in a distinct $U(3)$ space:

$$
\begin{aligned}
& B\left(\begin{array}{ccc} 
& \multicolumn{3}{c}{M_{11}^{\prime}} & \\
M_{13}^{\prime} & M_{12}^{\prime} & M_{22}^{\prime} \\
& M_{12} & M_{22}
\end{array}\right)
\end{aligned}
$$

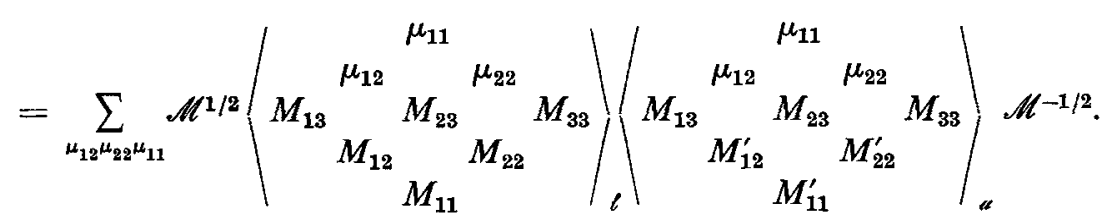

Here $\mathscr{M}$ is an invariant operator of $U_{n} * U_{n}$ which has eigenvalue equal to the measure $\mathscr{M}\left(M_{13} M_{23} M_{33}\right)$ for an arbitrary state vector with $U(3)$ irreducible labels 
$\left(M_{13} M_{23} M_{33}\right)$ and $B$ is the boson operator which brings the vacuum state of $U_{3} * U_{3}$ to a state with the assigned labels. That is,

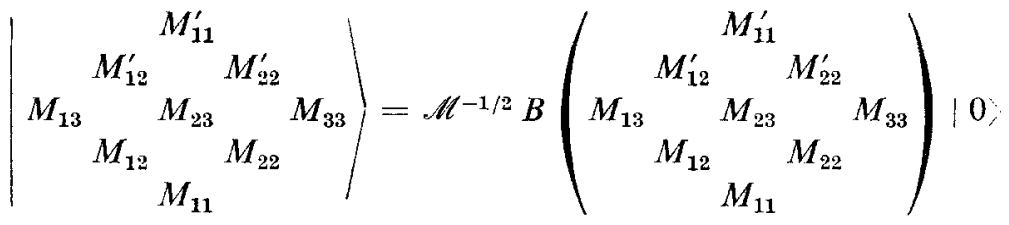

$$
\begin{aligned}
& \mathscr{H}=\frac{\left(M_{13}+2\right) !\left(M_{23}+1\right) ! M_{33} !}{\left(M_{13}-M_{23}+1\right)\left(M_{13}-M_{33}+2\right)\left(M_{23}-M_{33}+1\right)} \equiv \mathscr{M}\left(M_{13} M_{23} M_{33}\right) .
\end{aligned}
$$

The indices $t$ and $u$ designate the fact that the Wigner operators act, respectively, on the lower and upper Gelfand patterns of an arbitrary state vector of $U_{3} * U_{3}$ :

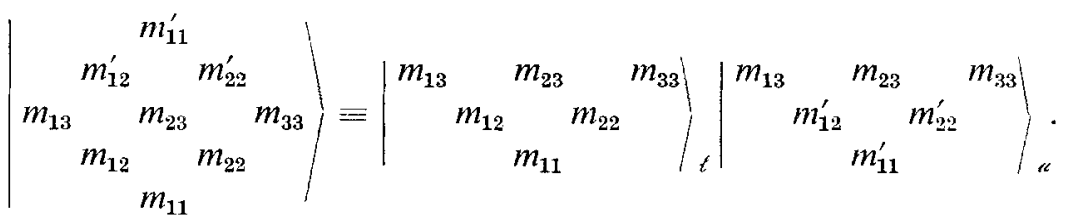

The crux of the multiplicity resolution is this: Upon choosing special $U(2)$ shifts in one $U(3)$ space, a single operator becomes nonvanishing, and the factorization lemma thereby determines this particular operator on general state vectors (in the second $U(3)$ space). Expressed somewhat differently, by choosing special initial and final state vectors in one $U(3)$-corresponding to maximal shift in the $U(2)$ subgroup-the multiplicity splits, that is, the operators branch off.

The actual methods of calculation are then the following.

First Method: $\langle 200\rangle \times\langle 220\rangle \rightarrow\langle 420\rangle$

We evaluate the Wigner operators for the $S U(3)$ representation $\langle 420\rangle$ from those of the representations $\langle 200\rangle$ and $\langle 220\rangle$ in a build-up fashion. Since these latter representations have no multiple weights, their Wigner operators can be easily obtained following Ref. [5], so we assume that they are known.

The first step is to express the boson (polynomial) operators of the representation $\left[\begin{array}{lll}4 & 2 & 0\end{array}\right]$ in terms of those of [ $\left[\begin{array}{lll}2 & 0 & 0\end{array}\right]$ and [ $\left[\begin{array}{lll}2 & 2 & 0\end{array}\right]$. These relations can be obtained by coupling together the boson operators of [ $\left[\begin{array}{lll}2 & 0 & 0\end{array}\right]$ and $\left[\begin{array}{lll}2 & 2 & 0\end{array}\right]$ to a resultant $\left[\begin{array}{lll}4 & 2 & 0\end{array}\right]$, using the fact that both lower and upper patterns of the boson polynomials are coupled by means of known Wigner coefficients (the $\langle 200\rangle$ and $\langle 220\rangle$ coefficients). 
We then have

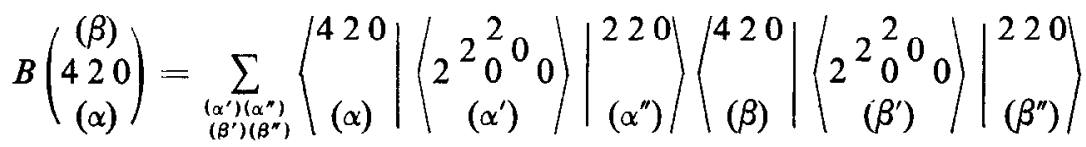

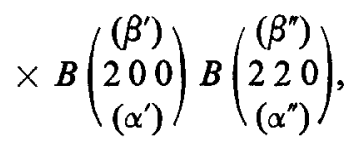

where we use an abbreviated notation: $(\alpha) \equiv\left({ }^{\alpha_{12}}{ }_{\alpha_{11}}{ }^{\alpha_{22}}\right)$.

As a second step we take Eq. (2.11) between initial and final state vectors of $U(n) * U(n)$, i.e., between

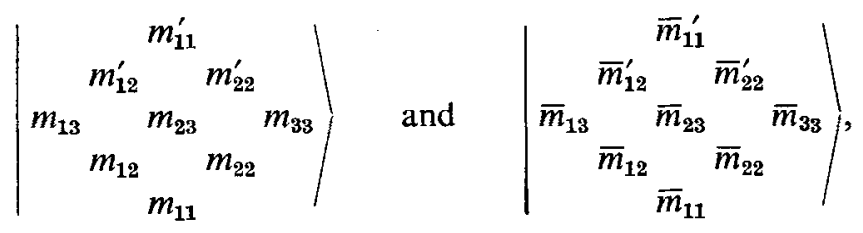

respectively.

Now apply the factorization lemma, Eq. (2.8) once on the left side of (2.11) and twice on the right side after expanding the product on a complete set of intermediate state vectors of $U(n) * U(n)$. In this way, we obtain an equation relating Wigner operators of $\langle 420\rangle$ to those of $\langle 200\rangle$ and $\langle 220\rangle$, namely,

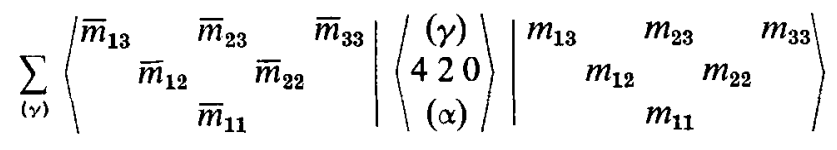

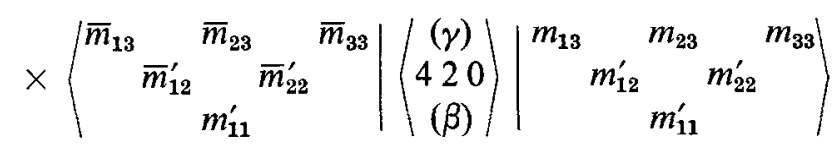

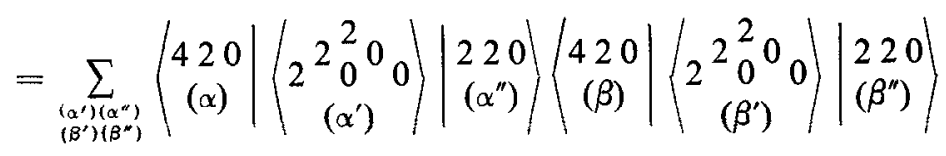

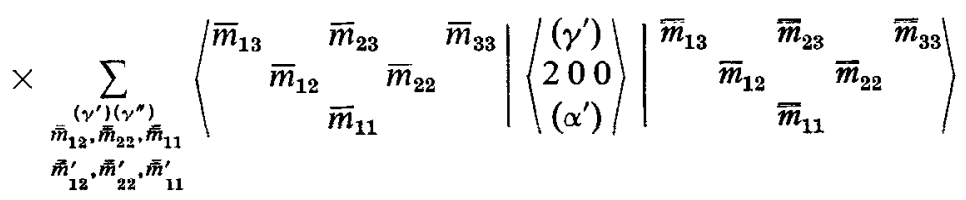




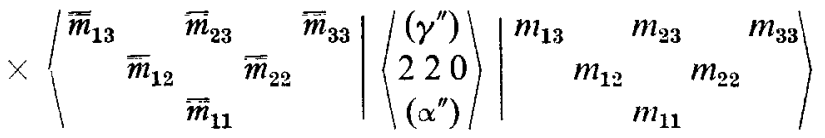

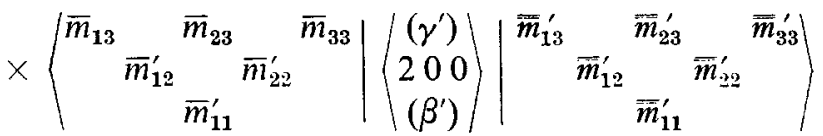

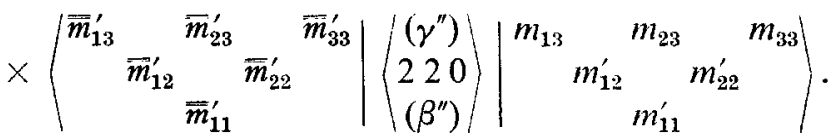

In view of the shifts $\Delta m_{i 3}$ induced by the Wigner operators (Eqs. 2.3) we have:

(1) The sum in $(\gamma)$ extends to all $(\gamma)$ such that

$$
\begin{aligned}
& \Delta m_{13}(\gamma)=\gamma_{11}=\bar{m}_{13}-m_{13}, \\
& \Delta m_{23}(\gamma) \equiv \gamma_{12}+\gamma_{22}-\gamma_{11}=\bar{m}_{23}-m_{23}, \\
& \Delta m_{33}(\gamma) \equiv 6-\gamma_{12}-\gamma_{22}=\bar{m}_{33}-m_{33} .
\end{aligned}
$$

(2) The sums in $\left(\gamma^{\prime}\right)$ and $\left(\gamma^{\prime \prime}\right)$ extend to all $\left(\gamma^{\prime}\right)$ and $\left(\gamma^{\prime \prime}\right)$ such that

$$
\begin{aligned}
\gamma_{11}^{\prime}+\gamma_{11}^{\prime \prime} & =\gamma_{11}, \\
\gamma_{12}^{\prime}+\gamma_{22}^{\prime}+\gamma_{12}^{\prime \prime}+\gamma_{22}^{\prime \prime} & =\gamma_{12}+\gamma_{22} .
\end{aligned}
$$

(3) The intermediate irreps should satisfy

$$
\begin{aligned}
& \bar{m}_{13}=\bar{m}_{13}^{\prime}=m_{13}+\gamma_{11}^{\prime \prime} \\
& \bar{m}_{23}=\bar{m}_{23}^{\prime}=m_{23}+\left(\gamma_{12}^{\prime \prime}+\gamma_{22}^{\prime \prime}-\gamma_{11}^{\prime \prime}\right) \\
& \bar{m}_{33}=\bar{m}_{33}^{\prime}=m_{33}+\left(4-\gamma_{12}^{\prime \prime}-\gamma_{22}^{\prime \prime}\right) .
\end{aligned}
$$

To find the explicit terms in the sum over $\bar{m}_{12}, \bar{m}_{22}, \bar{m}_{11} ; \bar{m}_{12}^{\prime}, \bar{m}_{22}^{\prime}, \bar{m}_{11}^{\prime}$ and to evaluate the resulting matrix elements of $S U(3)$ Wigner operators (i.e., $S U(3)$ Wigner coefficients) it is, of course, advantageous to express these matrix elements in terms of matrix elements of $U(2)$ Wigner operators (i.e., usual Clebsch-Gordan coefficients) and the matrix elements of the reduced Wigner operators (i.e., reduced Wigner coefficient or isoscalar factor). 
This is done by taking matrix elements of the reduction formula (Eq. 2.4), i.e.,

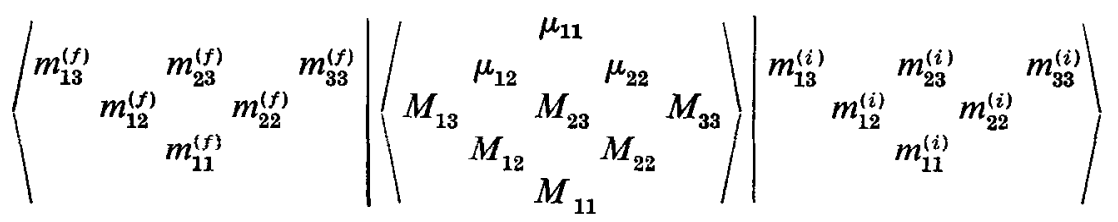

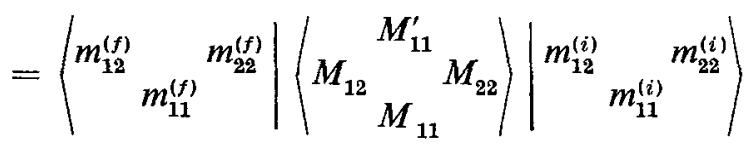

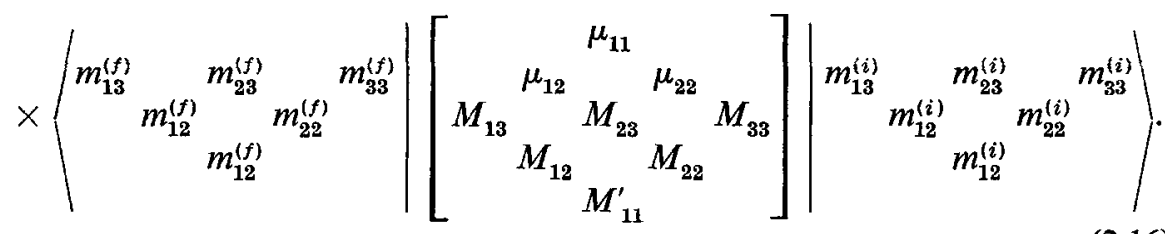

To simplify the writing of equations we will use the functional notation

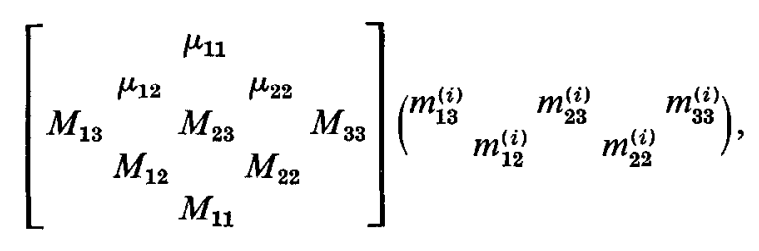

to designate the explicit matrix element of the reduced Wigner operators. In this notation the part $[\cdots]$ designates the function and the $(\cdots)$ designates the variables. This functional notation is completely explicit since the labels of the final $U(3)$ state vector in the matrix element are determined once we give those of the initial state vector, as can be seen in Eq. (2.5). (We have omitted $m_{11}^{(i)}$ since it must, by definition, be equal to $m_{12}^{(i)}$.)

Choosing appropriate values for the labels of the $U(3) * U(3)$ final state vector in (2.12), using (2.16) and the explicit expressions for the matrix elements of the reduced Wigner operators of $[200]$ and $[220]$ one gets an equation with the following structure:

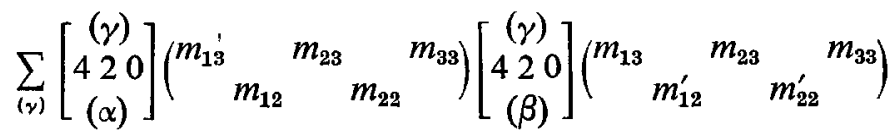

$$
\begin{aligned}
& =g(\alpha) g^{\prime}(\beta) \sum_{i} g_{i}(\alpha) g_{i}{ }^{\prime}(\beta) a_{i},
\end{aligned}
$$


where the $a_{i}$ depend only on $\left(m_{13}, m_{23}, m_{33}\right) ; g$ and $g^{\prime}$ are the same functions, the first having the variables $\left(m_{13}, m_{23}, m_{33}, m_{12}, m_{22}\right)$, and the second, the variables $\left(m_{13}, m_{23}, m_{33}, m_{12}^{\prime}, m_{22}^{\prime}\right)$. (The sum in $i$ comes from the fact that in general there are more than one pair $\left(\gamma^{\prime}\right)\left(\gamma^{\prime \prime}\right)$ satisfying (2.14).)

For the cases in which $\left(\bar{m}_{13}-m_{13}, \bar{m}_{23}-m_{23}, \bar{m}_{33}-m_{33}\right)$ is equal to a weight $\Delta$ of multiplicity one, both summations in Eq. (2.18) have only one term and the final answer has the factored form

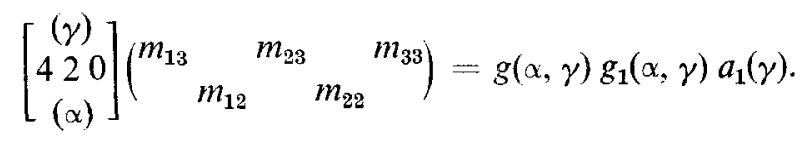

For those cases in which $\left(\bar{m}_{13}-m_{13}, \bar{m}_{23}-m_{23}, \bar{m}_{33}-m_{33}\right)$ is equal to $(2,2,2)$, that is, the cases with multiplicity three, the right side of $(2.18)$ has 6 terms and the triple degeneracy in the left side can be split by the arguments that follow.

When we compute the right side of $(2.18)$ for $\Delta(\gamma)=(222)$ and $(\alpha)=(\beta)=$ $\left({ }_{4}^{4}{ }^{0}\right)$, we find $g_{1}=g_{2}=g_{3}=1$ and $g_{4}=g_{5}=g_{6}=2$. With these values the right side factors completely, and assumes the form

$$
\left\{g(404)\left[\sum_{i=1}^{6} g_{i} a_{i}\right]^{1 / 2}\right\} \times\left\{\left[g^{\prime}(404)\left[\sum_{j=1}^{6} g_{j} a_{j}\right]^{1 / 2}\right\}\right.
$$

In other words the right side has the form of a function in $\left(m_{12} m_{22}\right)$ multiplied by the same function in $\left(m_{12}^{\prime} m_{22}^{\prime}\right)$. Since $\left(m_{12}, m_{22}\right)$ and $\left(m_{12}^{\prime}, m_{22}^{\prime}\right)$ are independent, and the three operators in the sum on the left side are linearly independent, this requires that only one of these three operators differ from zero, the others necessarily vanishing. We may then make the assignment of labels (precisely why is discussed below):

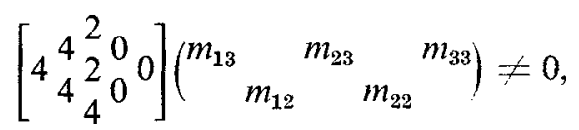

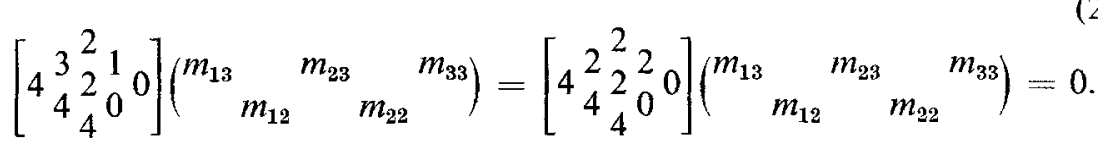

From this assignment, (2.21), and Eq. (2.18) we get the result

$$
\left[\begin{array}{l}
4 \underset{4}{4}{ }_{4}^{2} 0 \\
0
\end{array}\right]\left[\left(\begin{array}{lll}
m_{13} & m_{23} & m_{33}
\end{array}\right)=+g(404)\left[\sum_{i=1}^{6} g_{i} a_{i}\right]^{1 / 2}\right. \text {. }
$$


Using this value in Eq. (2.18), we get

$$
\left[\begin{array}{ccc}
4 & 4{ }^{2} & 0 \\
2 & 0
\end{array}\right]\left(\begin{array}{ccc}
m_{13} & m_{23} & m_{33} \\
& m_{12} & m_{22}
\end{array}\right)=\frac{g(\alpha)}{\left[\sum_{j=1}^{6} g_{j} a_{j}\right]^{1 / 2}} \sum_{i=1}^{6} g_{i}(\alpha) g_{i} a_{i}
$$

There are two arbitrary steps in this procedure: (a) the specific assignment of labels (Eq. 2.21), and (b) the choice of a \pm sign in extracting the square root (Eq. 2.22). When we discuss the limit properties (in Section 3), both steps will be seen as necessary in the light of a larger structure.

This procedure determines completely one of the three operators which induces a shift $\Delta_{3}=(222)$. Now we use (2.23) to eliminate one of the three terms on the left side of Eq. (2.18). This determines a new equation, involving now only two operators. Putting $(\alpha)=(\beta)=\left({ }_{4}^{4}{ }_{4}^{1}\right)$ in this new equation, once again we see that the right side splits into a product of two factors, one in the unprimed, the other in the primed variables. In other words, our previous argument may be iterated to determine successively the three operators having $\Delta=(222)$.

For the cases in which $\left(\bar{m}_{13}-m_{13}, \bar{m}_{23}-m_{23}, \bar{m}_{33}-m_{33}\right)$ is equal to a weight of multiplicity two, we use again the lower $U(2)$ labels $\left({ }_{4}{ }_{4}\right)$, and the same argument, used above, shows that the right side splits.

Second Method: $\langle 210\rangle \times\langle 210\rangle \rightarrow\langle 420\rangle$

In this method a buildup process is again used to construct Wigner operators of $U(3)$ irreducible tensor character $\langle 420\rangle$. There are two differences from the

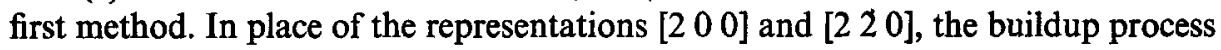
employs the representations [2 10 ] and [2 10 ]. In place of Boson (polynomial) operators, $U(3)$ Wigner operators of character $\langle 210\rangle$ are coupled directly to form an operator of irreducible tensor character $\langle 420\rangle$ :

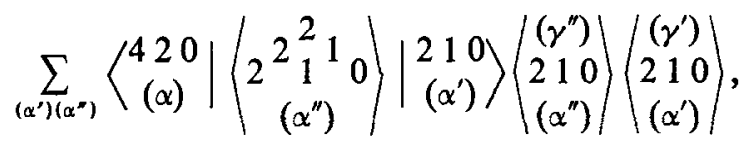

where $\left(\gamma^{\prime}\right),\left(\gamma^{\prime \prime}\right)$, and $(\alpha)$ are fixed. The matrix elements of such an operator between $U(3)$ state vectors $\langle(\bar{m})|$ and $|(m)\rangle$ can be expressed as a linear combination of Wigner operators which induce the shifts $\Delta m_{i 3}=\bar{m}_{i 3}-m_{i 3}(i=1,2,3)$ in the irrep labels of the initial state vector, since Wigner operators form a basis for tensor operators. The coefficients of such a linear combination must be $U(3)$ invariant, so

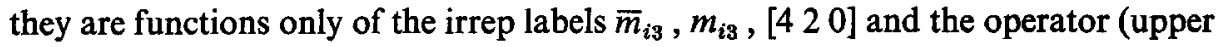
pattern) labels $(\gamma),\left(\gamma^{\prime}\right),\left(\gamma^{\prime \prime}\right),\left({ }_{2}^{2}\right)$. In fact these coefficients are precisely $U(3)$ Racah coefficients as can be seen by comparing the linear combination with the 
recoupling transformation involving three $U(3)$ irreducible representations-the classical way of defining Racah coefficients.

Taking matrix elements of $(2.24)$ between $U(3)$ state vectors $\langle(\bar{m})|$ and $|(m)\rangle$ one thus gets

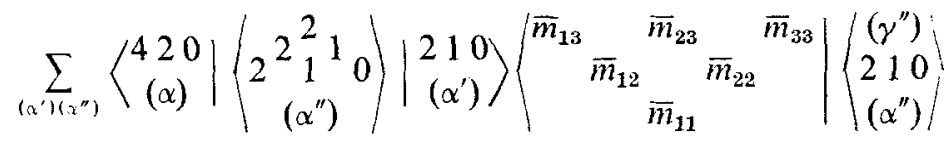

$$
\begin{aligned}
& \times\left|\begin{array}{ccc}
\bar{m}_{13} & \bar{m}_{23} & \bar{m}_{33} \\
\bar{m}_{12} & \bar{m}_{22} \\
& \bar{m}_{11}
\end{array}\right|
\end{aligned}
$$

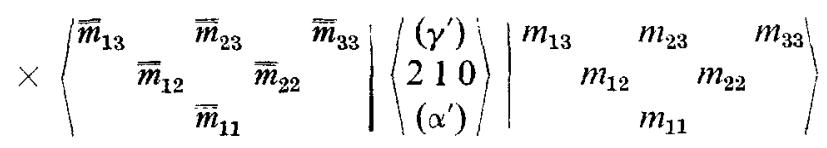

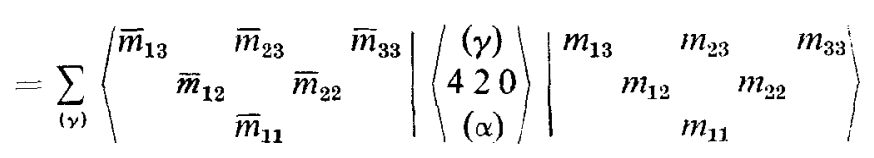

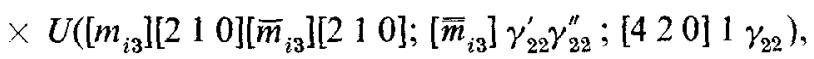

where the sum in $(\gamma)$ extends to all $(\gamma)$ such that $\Delta(\gamma)_{i}=\bar{m}_{i 3}-m_{i 3}$ and the $U$ "coefficients" are $U(3)$ Racah coefficients in unitary form. [Our notation for the Racah coefficients is a generalization of the notation of ordinary angular momentum calculus; the order of the $U(3)$ irrep symbols is that introduced by Racah. The row and column indices in the Racah coefficients must include the operator (upper pattern) labels $\left(\gamma^{\prime}\right),\left(\gamma^{\prime \prime}\right),(\gamma)$ and $\left(\gamma^{\prime \prime \prime}\right) \equiv\left({ }_{2}{ }^{2}{ }_{1}\right)$; but since $m_{i 3}, \bar{m}_{i 3}$ and $\bar{m}_{i 3}$ are fixed, we see (by Eq. (2.3)) that, in the problem we are dealing with, the labels $\gamma_{22}$ are sufficient to characterize the multiplicities, when present.]

By writing a second equation of the form (2.25) with $(\alpha)$ 's replaced by $(\beta)$ 's, multiplying the two equations, summing over $\left(\gamma^{\prime}\right)$ and $\left(\gamma^{\prime \prime}\right)$ (hence also $\overline{\bar{m}}_{i 3}$ ), and using the orthonormality relations of the Racah coefficients, an equation of the form (2.12) is obtained. The present method however, makes use of Eq. (2.25) directly and therefore leads to a simultaneous calculation of the $U(3)$ Wigner and Racah coefficients. (Explicit tabulations of the matrix elements of the operators [ $\left.\begin{array}{lll}2 & 1 & 0\end{array}\right]$ needed for the evaluation of (2.25) are given in Refs. [1c] and [6].)

In cases where the $\left(\bar{m}_{i 3}-m_{i 3}\right)$ do not lead to multiplicities, the single $U$-coefficient that then appears in Eq. (2.25) serves merely as a normalization constant. Its magnitude can be evaluated from the orthonormality relations of the $U(3)$ Wigner operators. 
In cases where there are multiplicities, this can be resolved by special choice of $\left(\gamma^{\prime}\right)$ and $\left(\gamma^{\prime \prime}\right)$. This can be seen by using some special features of the $U(3)$ Wigner operator [2 10 ]. The $U(3)$ Wigner operators $\left\langle\begin{array}{lll}2 & 1 & 0\end{array}\right\rangle$ with operator labels $(\gamma)=\left({ }_{1}{ }_{1}{ }_{1}\right),\left(\left\langle{ }_{2} 1_{1}^{1} 1_{0}\right\rangle\right)$ is identified with the generators of the group [1c, 6]. As can be seen from the expressions of its matrix elements [1c, 6], the maximum change in the isospin $\left(I=1 / 2\left(m_{12}-m_{22}\right)\right)$ that a $S U(3)$ generator can produce is $|\Delta I|=1 / 2$. Therefore the reduced Wigner operators of $\left\langle a_{2} 1_{1}^{1} 1_{0}\right\rangle$ with $|\Delta I|>1 / 2$

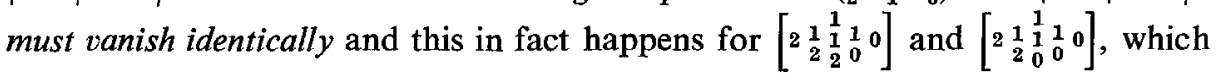
produce $\Delta I=1$ and $\Delta I=-1$, respectively.

For the multiplicity three cases $(\gamma)=\left(\mathrm{a}_{2}{ }_{2}\right)$, and therefore $\left(\gamma^{\prime}\right)$ and $\left(\gamma^{\prime \prime}\right)$ can independently be $\left(1_{1}{ }_{1}\right)$ or $\left({ }_{2}{ }^{1}{ }_{0}\right)$. With the choice $\left(\gamma^{\prime}\right)=\left(\gamma^{\prime \prime}\right)=\left({ }_{1}{ }_{1}{ }_{1}\right)$ the operator (2.24) has the property that its reduced Wigner operators with Gelfand labels (lower pattern) $(\alpha)=\left({ }^{4}{ }_{4}{ }^{0}\right),\left({ }^{4}{ }_{0}{ }^{0}\right),\left({ }^{4}{ }_{3}{ }^{0}\right),\left({ }^{4}{ }_{1}{ }^{0}\right)$ and similarly $(\alpha)=\left({ }^{4}{ }_{4}{ }^{1}\right),\left({ }^{4}{ }_{1}{ }^{1}\right)$; $\left({ }_{3}^{3}{ }^{0}\right),\left({ }^{3} 0_{0}^{0}\right)$ are all equal to zero. [The first of these has the components $2,-2,1$, -1 of an $I$-spin tensor of rank 2 while the second has the components of two I-spin tensors of rank 3/2.] This property allows us to identify the operator (2.24) resultant from this choice as the operator $\left\langle\left\langle_{4} 2_{2}^{2} 2_{0}\right\rangle\right.$ of the first method. Then the sum over $(\gamma)$ in the right side of (2.25) collapses to a single term, and the calculation proceeds as for a matrix element without multiplicities. With the choice $\left(\gamma^{\prime}\right)=\left({ }_{1}{ }_{1}\right),\left(\gamma^{\prime \prime}\right)=\left({ }_{2}{ }_{0}{ }_{0}\right)$ it would appear from similar arguments that the matrix elements of Eq. (2.25) would involve a combination of matrix elements of Wigner operators $\left\langle 4_{4} \mathbf{3}_{2}^{2} 1_{0}\right\rangle$ and $\left\langle 4_{4} 2_{2}^{2} 2_{0}\right\rangle$. In this case, however, the symmetry under conjugation parity (Symmetry 1 of Section 3) rules out $\left\langle\alpha_{4} z_{2}^{2} z_{0}\right\rangle$ and the calculation of the matrix elements of the operator $\left\langle 4_{3}{ }_{2}^{2} 1_{0}\right\rangle$ proceeds as for a matrix element without multiplicities. With the choice $\left(\gamma^{\prime}\right)=\left(\gamma^{\prime \prime}\right)=\left({ }_{2}{ }^{1}{ }_{0}\right)$, the matrix elements of Eq. (2.25) involve a combination of matrix elements of Wigner operators $\left\langle\left\langle_{4} 4_{2}^{2} 00\right.\right.$ and $\left\langle 4_{4} 2_{2}^{2} 2_{0}\right\rangle$, both with the same conjugation parity. Since we already know one of them, the other $\left(\left\langle\left\langle_{4} 4_{2}^{2} 0_{0}\right\rangle\right)\right.$ can be calculated using the orthonormality relations for Wigner operators.

To resolve the cases of multiplicity two we observe in Fig. 1 that the positions of the double degenerate weights coincide with the positions of the single weights of the analogous figure for the representation [2 10 ]. This tells us that for the multiplicity two cases $\left(\gamma^{\prime}\right)$ and $\left(\gamma^{\prime \prime}\right)$ should be chosen such that one of them is equal to $\left({ }_{1}{ }_{1}\right)$ or $\left({ }_{2}{ }_{0}\right)$ and the other is equal to any $(\gamma)$ different from $\left({ }_{1}{ }_{1}\right)$ and $\left({ }_{2}{ }_{0}\right)$ (the ones leading to single weights in the wcight diagram of [2 10$]$ ). The rest proceeds in the same way as the multiplicity three cases.

Since this method essentially calculates only products of Wigner and Racah coefficients, these are again determined only to within an overall \pm sign which is fixed by the limit properties to be discussed in the next section. 


\section{Some General Properties of the Matrix Elements}

The matrix elements of the reduced Wigner operators all have the general form

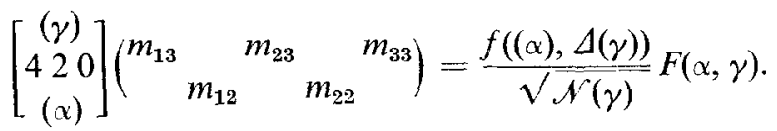

The function $f$ is a square root of a product of linear factors. Up to a numerical factor, it can be obtained directly from the "pattern calculus" [5]. Note that $f$ depends on $(\gamma)$ only through the weight (i.e., shift) of the upper (operator) pattern. [That is, operators with different $(\gamma)$ but the same $\Delta(\gamma) \equiv\left(\gamma_{11}, \gamma_{12}+\gamma_{22}-\gamma_{11}\right.$, $\left.6-\gamma_{12}-\gamma_{22}\right)$ have for the same $(\alpha)$ the same factor $f$.]

The function $\mathscr{N}$ is a normalization factor, and depends only on the $U(3)$ irrep labels $\left(m_{13}, m_{23}, m_{33}\right)$. The function $\mathscr{N}$ may (and indeed must) vanish for certain (lexical) choices of the variables $\left\{m_{i 3}\right\}$. For such a case the numerator of Eq. (3.1) also vanishes, but we nonetheless must, for consistency, define the operator to vanish when operating on state vectors having these labels.

[This vanishing of the normalization function $\mathscr{N}$ reflects an important structural property of the canonical splitting of the multiplicity. It is a necessary property that when $\langle 420\rangle$ acts on certain irreps the actual multiplicity is less than the maximum. (This is not unfamiliar; for example, all operators acting on $\left.\left[m_{13} 00\right]\right\rangle$ state vectors necessarily have multiplicity one.) In every case where the actual multiplicity is not maximal, one or more of the normalization functions will necessarily vanish. We may express this by saying that the vanishing of $\mathscr{N}$ determines the $n u l l$ space belonging to the associated operator [7].]

The function $F$ is a polynomial in the five $S U(3)$ labels, $m_{13}, m_{33}, m_{33} ; m_{12}, m_{23}$.

The methods of Section 2 determine the reduced Wigner operators up to an overall phase; in addition, there is a choice in assigning upper (operator) labels to the operators with multiplicity. To solve these problems we make use of an important asymptotic limit requirement on the matrix elements of reduced Wigner operators [7], namely,

$$
\begin{aligned}
& \lim _{m_{22} \rightarrow-\infty} \lim _{m_{33} \rightarrow-\infty}\left(\left[\begin{array}{c}
(\gamma) \\
420 \\
(\alpha)
\end{array}\right]\left(\begin{array}{llll}
m_{13} & & m_{23} & \left.m_{33}\right) \\
& m_{12} & m_{22}
\end{array}\right)\right) \\
& =\delta_{\alpha_{12}}^{\gamma_{12}} \delta^{\gamma_{22}} \cdot\left[\begin{array}{cc}
\alpha_{22} & \gamma_{11} \\
\alpha_{12} & \alpha_{22} \\
& \alpha_{11}
\end{array}\right]\left(\begin{array}{lll}
m_{13} & & m_{23} \\
& m_{12}
\end{array}\right) .
\end{aligned}
$$

This formula, due to the presence of the delta function factors, tells us that in the limit of (negatively) large $m_{22}$ and $m_{33}$ the matrix element of the reduced Wigner 
operators must vanish unless the $U(2)$ irrep labels of the operator pattern $\left(\gamma_{12}, \gamma_{22}\right)$ coincide with those of the lower pattern $\left(\alpha_{12}, \alpha_{22}\right)$. This property, for the case of multiple operators, determines uniquely which upper labels must be assigned to the operators.

When the labels match properly, the right side of (3.2) gives us an $S U(2)$ Wigner (Clebsch-Gordan) coefficient in the variables $\left(m_{13}, m_{23}, m_{12}\right)$. [For $U(2)$ the reduced Wigner operator (denoted by square brackets) coincides with the Wigner operator itself (angular brackets). We chose to use square brackets in Eq. 3.2 to indicate that this is a special case of a more general property.]

By comparing with the asymptotic limit of the operator on the left side, we also determine the correct overall phase of the operator.

The matrix elements of the reduced Wigner operators have the following symmetries:

(1) The Symmetry Related to Conjugation

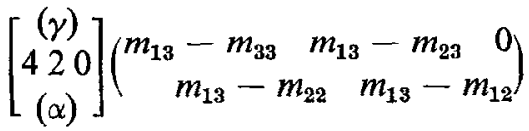

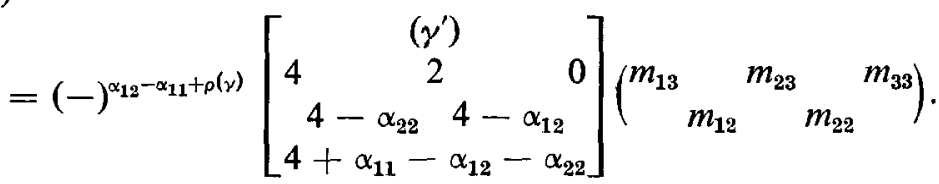

Here $(-)^{o(\gamma)}$ is an overall sign for all the components of an operator with upper labels $(\gamma)$. (It is just the parity of the operator under conjugation.) For the multiplicity three cases it coincides with $(-)^{\gamma_{22}}$.

It is quite difficult to give an explicit formula relating $\left(\gamma^{\prime}\right)$ to $(\gamma)$. From $(\gamma)$ one knows directly the shifts it induces on the initial irrep labels. That is, $(\gamma) \rightarrow \Delta(\gamma)$. Similarly, we know that $\Delta\left(\gamma^{\prime}\right)$ is known from $\Delta(\gamma)$; this is just the operation of conjugation applied to the shift labels: $\Delta\left(\gamma^{\prime}\right)=\widetilde{\Delta}(\gamma)$. Explicitly,

$$
\begin{aligned}
\Delta\left(\gamma^{\prime}\right)_{13} & =\Delta(\gamma)_{13}-\Delta(\gamma)_{33}+\chi, \\
\Delta\left(\gamma^{\prime}\right)_{23} & =\Delta(\gamma)_{13}-\Delta(\gamma)_{23}+\chi, \\
\Delta(\gamma)_{33} & =\chi .
\end{aligned}
$$

[Here $\chi=4-\gamma_{11}$ is a "translation factor" to make $\tilde{\Delta(\gamma)}$ an allowed weight of (4 $\left.\begin{array}{ll}4 & 0\end{array}\right)$.]

If one could obtain $\left(\gamma^{\prime}\right)$ from $\Delta\left(\gamma^{\prime}\right)$, we could then find the induced transformation:

$$
\begin{gathered}
(\gamma) \leadsto\left(\gamma^{\prime}\right) \\
\downarrow \\
\Delta(\gamma) \rightarrow \delta^{\uparrow}(\gamma) .
\end{gathered}
$$


However, only in the absence of multiplicity is this association (a priori) unique. The canonical splitting of the multiplicity makes the association always unique.

Using the canonical labelling, we first partition the 27-plet operators into three classes:

Class I contains the 19 operators with operator labels:

$$
\begin{gathered}
(\gamma)=\left(\begin{array}{c}
4 \\
42
\end{array}\right),\left(\begin{array}{c}
3 \\
42
\end{array}\right),\left(\begin{array}{c}
2 \\
42
\end{array}\right),\left(\begin{array}{c}
4 \\
41
\end{array}\right),\left(\begin{array}{c}
1 \\
41
\end{array}\right),\left(\begin{array}{c}
4 \\
40
\end{array}\right),\left(\begin{array}{c}
0 \\
40
\end{array}\right),\left(\begin{array}{c}
3 \\
30
\end{array}\right),\left(\begin{array}{c}
0 \\
30
\end{array}\right),\left(\begin{array}{c}
2 \\
20
\end{array}\right),\left(\begin{array}{c}
1 \\
20
\end{array}\right),\left(\begin{array}{c}
0 \\
20
\end{array}\right), \\
\left(\begin{array}{c}
3 \\
41
\end{array}\right),\left(\begin{array}{c}
2 \\
41
\end{array}\right),\left(\begin{array}{c}
3 \\
40
\end{array}\right),\left(\begin{array}{c}
1 \\
40
\end{array}\right),\left(\begin{array}{c}
2 \\
30
\end{array}\right),\left(\begin{array}{c}
1 \\
30
\end{array}\right) \\
\left(\begin{array}{c}
2 \\
40
\end{array}\right)
\end{gathered}
$$

Class II contains the 7 operators with operator labels:

$$
\begin{gathered}
(\gamma)=\left(\begin{array}{c}
3 \\
32
\end{array}\right),\left(\begin{array}{c}
2 \\
32
\end{array}\right),\left(\begin{array}{c}
3 \\
31
\end{array}\right),\left(\begin{array}{c}
1 \\
31
\end{array}\right),\left(\begin{array}{c}
2 \\
21
\end{array}\right),\left(\begin{array}{c}
1 \\
21
\end{array}\right), \\
\left(\begin{array}{c}
2 \\
31
\end{array}\right) .
\end{gathered}
$$

Class III contains only the operator with operator labels: $(\gamma)=\left({ }_{2}^{2}{ }_{2}\right)$.

The canonical splitting requires that this symmetry operation not mix classes.

This symmetry is an extension to the reduced matrix elements of the conjugation operation applied to the (complete) Wigner coefficient, i.e.,

$$
\left\langle(m)_{1}(m)_{2} \mid(m)_{3}\right\rangle \rightarrow(-)^{\circ}\left\langle(\tilde{m})_{1}(\tilde{m})_{2} \mid(\tilde{m})_{3}\right\rangle .
$$

For the $U(2)$ Wigner coefficients this symmetry is precisely the symmetry under change of sign of the magnetic quantum numbers.

(2) Reflection in the Hypercharge Axis

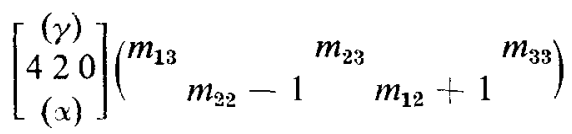

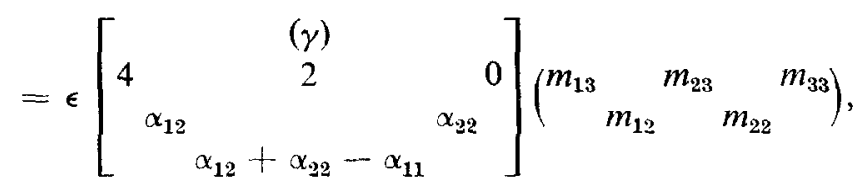


where $\epsilon$ is a plus or minus sign. For the multiplicity three cases we have $\epsilon=(-)^{\alpha_{12}+\alpha_{22}+\alpha_{11}\left(\delta_{Y^{2}}^{4}-1\right)}$. (For the other cases the phase $\epsilon$ is very complicated and will be omitted.)

(3) Symmetry Under Permutation of the U(3) Partial Hooks

$P_{i j}\left\{\left[\begin{array}{c}(\gamma) \\ 42 \\ (\alpha)\end{array}\right]\left(\begin{array}{cccc}m_{13} & & m_{23} & m_{33} \\ & m_{12} & m_{22}\end{array}\right)\right\}=\epsilon\left(\alpha, \gamma, \gamma^{\prime}\right)\left[\begin{array}{c}\left(\gamma^{\prime}\right) \\ 420 \\ (\alpha)\end{array}\right]\left(\begin{array}{llll}m_{13} & & m_{23} & m_{33} \\ & & m_{12} & m_{22}\end{array}\right)$

where $P_{i j}$ is the permutation $p_{i 3} \leftrightarrow p_{j 3}$ in the matrix element. (The partial hooks are defined by: $p_{i j} \equiv m_{i j}+j-i$.)

The relation between $(\gamma)$ and $\left(\gamma^{\prime}\right)$ is analogous to the one in the conjugation symmetry, the only difference being that now the shifts $\Delta(\gamma)_{3}$ and $\Delta\left(\gamma^{\prime}\right)_{3}$ are related by a permutation in the $\Delta_{i 3}$. That is, $\Delta\left(\gamma^{\prime}\right)_{i 3}=\Delta(\gamma)_{j 3}, \Delta\left(\gamma^{\prime}\right)_{j 3}=\Delta(\gamma)_{i 3}$, the other remains unaltered.

For the multiplicity three cases we have $\left(\gamma^{\prime}\right)=(\gamma)$ and $\epsilon\left(\alpha, \gamma, \gamma^{\prime}\right) \equiv 1$. This means that the matrix elements of these reduced Wigner operators are invariant under all permutations of the $\left\{p_{i 3}\right\}$. Then, by the fundamental theorem on invariants [8] they can be expressed in terms of the symmetric variables: the two Casimir $S U(3)$ invariants and the $U(2)$ variables $I$ (isospin) and $Y$ (hypercharge). (These are the four (Elliott) variables $g, \Gamma, \epsilon, \Lambda$-see the notation guide in Appendix II.)

For the other cases the values of $\epsilon\left(\alpha, \gamma, \gamma^{\prime}\right)$ are given in Table XI for the entries $(\alpha),(\gamma),\left(\gamma^{\prime}\right), i, j$.

This symmetry was recently studied by Alisauskas and Jucys [9].

(4) Symmetry Under Permutation of the Representations Involved

The $S U(3)$ Wigner coefficients have also definite symmetries under permutations of the representations involved [6]. We present here only that permutation which takes the $\langle 420\rangle$ operator into itself. For this we find

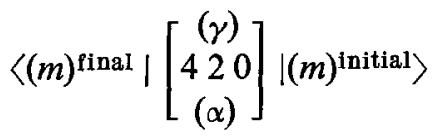

$$
\begin{aligned}
& =(-)^{\alpha_{12}+\alpha_{22}-\alpha_{11}}\left[\frac{\operatorname{dim}\left(\left[m_{i 3}{ }^{f \text { final }}\right)\left(p_{12}-p_{22}\right)^{\text {initial }}\right.}{\operatorname{dim}\left(\left[m_{i 3}\right]^{\text {initial }}\right)\left(p_{12}-p_{22}\right)^{\text {final }}}\right]^{1 / 2}
\end{aligned}
$$

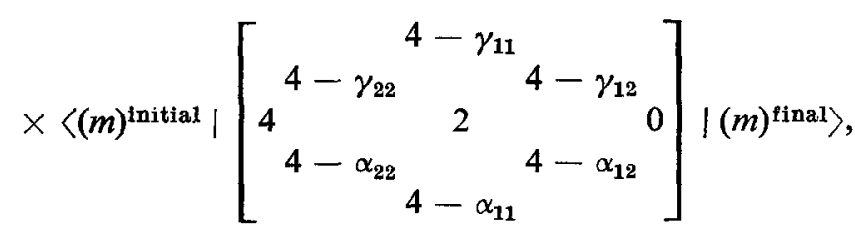


where

$$
\begin{aligned}
\operatorname{dim}\left(\left[m_{i 3}\right]\right) & =1 / 2\left(m_{13}-m_{23}+1\right)\left(m_{13}-m_{33}+2\right)\left(m_{23}-m_{33}+1\right) \\
& =1 / 2\left(p_{13}-p_{23}\right)\left(p_{13}-p_{33}\right)\left(p_{23}-p_{33}\right) \\
& =1 / 2(\lambda+1)(\mu+1)(\lambda+\mu+2) .
\end{aligned}
$$

\section{Applications}

\section{A. Particle Physics}

The present situation in particle physics has reached something of a consensus: unitary symmetry is to be confined almost exclusively to singlets and octets for mesons; and unitary singlets, octets and decimets for baryons. The justification for this restriction is entirely empirical; it has, however, been raised to the status of an axiom ("the absence of exotic resonances") in the so-called duality diagrams. For the present work this has a clear implication: the 27-plet is essentially of no interest as a possible particle multiplet-although, to be sure, there are early papers to the contrary.

Unitary symmetry is, however, strongly broken and implies that the particle wave functions must contain components from other multiplets, preserving, however, $I$ and $Y$. It is usually not possible to estimate this mixing, since a dynamical basis for particle physics is lacking. Admixture of 27-plet components into the baryon decimet was proposed by Cutkosky [10] to explain the reduction of the relative width $\Gamma(\Sigma(1385) \rightarrow \Sigma+\pi) / \Gamma(\Sigma(1385) \rightarrow \Lambda+\pi)$ from $0.16(S U(3)$ value $)$ to the experimental value $\cong 0.04$. Cutkosky estimated the mixing to be expected on a self-consistent (bootstrap) model of the baryon states. Wali and Warnock [11] and Golowich [12] show that 27-plet mixing is less important than might be expected [10b].

As a unitary operator, however, the 27-plet system is of considerable current interest. Applications of the 27-plet operator occur in several basic ways:

(a) Second order terms in the Gell-Mann-Okubo mass formula [13].

(b) Electromagnetic mass shifts [10b].

(c) Two photon operators [10b]

(d) Weak intcractions ( $\Delta I=3 / 2,27$-plet piece of the weak Hamiltonian) [14].

(e) Chiral $(S U(3) \times S U(3))$ symmetry breaking [15].

As an illustration of the applicability of the present results, let us consider in more detail item (a). In terms of unitary operators, the celebrated Gell-MannOkubo mass formula can be considered as the statement that the medium strong interactions which break unitary symmetry transform as the $I=Y=0$ component 


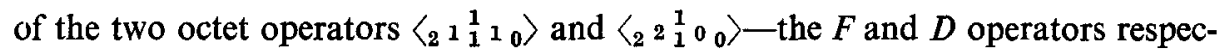
tively. To first order, then, the mass operator has the form

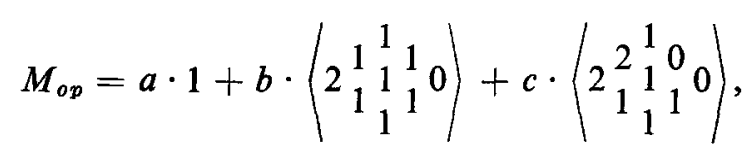

from which we obtain the general algebraic form of the Gell-Mann-Okubo relation

$$
M=a+b \cdot \frac{1 / 2 Y}{\sqrt{I_{2}}}+\frac{c}{\sqrt{B}} \cdot\left(I(I+1)-\left(\frac{Y}{2}\right)^{2}-I_{2}-\frac{3 I_{3}}{I_{2}} Y\right),
$$

with $B \equiv\left(4 I_{2}{ }^{3}+I_{2}{ }^{2}-36 I_{3}{ }^{2}\right) / I_{2}$.

[Here $I_{2}$ and $I_{3}$ are the second and third order Casimir invariants given in Appendix II.]

If we specialize to the octet case, our general result for the Gell-Mann-Okubo formula takes the form

$$
M=a+b \cdot \frac{Y}{2}+\frac{c}{\sqrt{5}}\left(I(I+1)-\left(\frac{Y}{2}\right)^{2}-1\right) .
$$

This result-and hence our general result-differs from the usual version of the Gell-Mann-Okubo formula in two ways: (a) The operators have been properly normalized (this is the origin of the complicated normalization factor, $B$, above); (b) the operators have been given precise (covariant) meaning [16] (note the extra " -1 " in the last term compared to the usual form of the Gell-Mann-Okubo result).

Both changes are essential in the Gell-Mann-Okubo formula, if we are going to compare the symmetry breaking terms between various unitary multiplets, and if we are to compare the actual size of the various terms arising in different orders.

Let us now extend these results to the next order. Estimates of the size of the second order effect of the symmetry breaking in the Gell-Mann-Okubo mass formula-specialized to the octet-were first carried out by Okubo [13] and by Dalitz [17]. Since the separation of properly covariant tensor operators has not been taken explicitly into account in this, or subsequent work, it is of interest to indicate these changes here.

To second order in the medium strong interaction (and using normalized covariant operators), one finds for the mass formula the form

$$
\begin{aligned}
& M_{o p}=a \cdot 1+b \cdot\left\langle\begin{array}{llll}
1 & 1 & 1 \\
2 & 0 & 0 & 0 \\
1 & 1 & 1
\end{array}\right)+c\left\langle\begin{array}{cccc}
2 & 1 & 0 \\
2 & 1 & 0 & 0 \\
1 & 1 & 0
\end{array}\right\rangle
\end{aligned}
$$

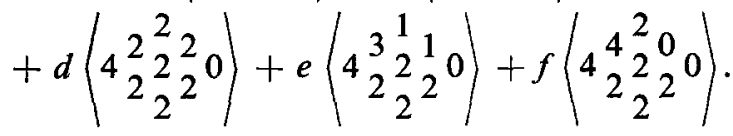


The general matrix elements of this operator can be written down from the tables of Section 5, but we shall specialize at once to octets and decimets. For both of these cases only one 27-plet operator is nonvanishing and we get, for the octet, the mass formula

$$
\begin{aligned}
M= & a \cdot 1+b \cdot \frac{Y}{2}+\frac{c}{\sqrt{5}} \cdot\left[I(I+1)-\left(\frac{Y}{2}\right)^{2}-1\right] \\
& +d \cdot \frac{1}{\sqrt{5}}\left[Y^{2}+\frac{4}{9} I(I+1)-1\right] .
\end{aligned}
$$

(It is interesting to note that just these four operators form orthonormal vectors (with respect to the trace) over the four $I, Y$ multiplets of the octet.)

Applying this to the baryon octet we find

$$
\begin{aligned}
& a_{8}=(1150.18 \pm 0.12) \mathrm{MeV} \\
& b_{8}=(379.07 \pm 0.36) \mathrm{MeV} \\
& c_{8}=(89.01 \pm 0.12) \mathrm{MeV} \\
& d_{8}=(-11.87 \pm 0.20) \mathrm{MeV}
\end{aligned}
$$

These terms show the expected variation in order of magnitude, in particular the second-order effect, $d_{8}$, is very small. Electromagnetic splittings are of the order of $d_{8}$ so we must correct $d_{8}$ for such effects. We can do this most expediently by the very nice technique (of Dalitz [17]) which uses as the $(I, Y)$ multiplet average masses the values

$$
\begin{gathered}
M(I=1 / 2, Y=1)=1 / 2\left(M_{P}+M_{N}\right), \\
M(I=1, Y=0)=M_{\Sigma^{+}}+M_{\Sigma^{-}}-M_{\Sigma^{0}}, \\
M(I=0, Y=0)=M_{A}, \\
M(I=1 / 2, Y=-1)=1 / 2\left(M_{\Xi^{0}}+M_{\Xi^{-}}\right) .
\end{gathered}
$$

For this choice all electromagnetic effects cancel out in determining $d_{8}$. The corrected value of $d_{8}$ is found to be $d_{8}=-12.34 \pm 0.40 \mathrm{MeV}$. This is only slightly changed from the previous value and shows that the 27 -plet contribution is probably genuine (but small) and nonelectromagnetic in origin.

Specializing next the general mass formula to the decimet, the $D$ operator, $\left\langle\begin{array}{lll}2 & \begin{array}{l}1 \\ 1\end{array} & 0\end{array}\right\rangle$, vanishes, along with two of the 27 -plet operators, and we find

$$
M=a+b \cdot \frac{Y}{2 \sqrt{2}}+d \cdot \frac{1}{9 \sqrt{7}}\left(5 Y^{2}+3 Y-5\right) .
$$


Applying this to the $\frac{3}{2}^{+}$decimet one finds

$$
\begin{aligned}
& a_{10}=1383.6 \mathrm{MeV}, \\
& b_{10}=-415.3 \mathrm{MeV}, \\
& d_{10}=-6.43 \mathrm{MeV} .
\end{aligned}
$$

The second-order term, $d_{10}$, is quite small and of the order of the electromagnetic splittings. We can correct for these effects by including $D_{8}$ and (27) $)_{8}$ terms, along the $Q$-direction; that is,

$$
M=a+b \frac{Y}{2 \sqrt{2}}+d \frac{5 Y^{2}+3 Y-5}{9 \sqrt{7}}+e \frac{Q}{2 \sqrt{2}}+f \frac{5 Q^{2}+3 Q-5}{9 \sqrt{7}} .
$$

Again applying this to the ${\frac{3^{+}}{2}}^{+}$decimet we find

$$
\begin{aligned}
& a_{10}=1381.4 \mathrm{MeV}, \\
& b_{10}=-416.5 \mathrm{MeV}, \\
& d_{10}=-17.1 \mathrm{MeV}, \\
& e_{10}=-14.1 \mathrm{MeV}, \\
& f_{10}=15.9 \mathrm{MeV}
\end{aligned}
$$

We note that $a_{10}$ and $b_{10}$ are essentially unaffected, in contrast to the term $d_{10}$. We conclude that the second-order effects are probably not significantly determined by data of the present accuracy.

It would be reasonable now to apply the second-order formula to the pseudoscalar and the vector meson octets. The situation, however, is not very clear: If one uses the Gell-Mann-Okubo approach (extended to the 27-plet), singlet-octet mixing is not included; if one uses the quark model approach (with the lambda quark heavier) singlet-octet mixing occurs very naturally, but then the model fails to give the (baryon) Gell-Mann-Okubo formula and excludes 27-plet corrections. A purely phenomenological approach is under-determined. We will not pursue this further except to say that the vector meson octet, with singlet-octet mixing, shows a very small 27 -plet contribution (less than $1 \%$ of the central (mass) ${ }^{2}$ term).

It should be remarked that the tables of $S U(3)$ recoupling coefficients are directly of interest in particle physics as crossing matrices. The results presented in Appendix I are a considerable extension of previously given tables.

\section{B. Nuclear Shell Theory}

In shell model calculations of light nuclei, in particular in the $2 s-1 d$ and $1 p$ shell, a classification of many-nucleon states according to representations of $S U(3)$ leads to a physically meaningful truncation of the shell model space. Unfortunately 
the relevant classification scheme leads to the group chain $S U(3) \supset R(3)$ where $R(3)$ refers to the orbital angular momentum $(L)$ of the shell model basis. The Wigner coefficients for the canonical group chain $S U(3) \supset S U(2)$ therefore have few direct, immediate applications in nuclear structure problems. The calculation of nuclear matrix elements by fractional parentage techniques can, however, be simplified by performing sums over the $S U(3)$ subgroup labels. The resultant expressions involve $S U(3)$ Racah coefficients. Since these are independent of the subgroup chain and are a natural byproduct of the calculation of $S U(3)$ Wigner coefficients, the results of the present work can be used in applications to shell model calculations.

A totally antisymmetric $n$-nucleon wave function can be expanded in totally antisymmetric functions for $(n-1)$ particles coupled to an $n$-th one by an expansion in terms of coefficients of fractional parentage (cfp). The cfp can be factored into a space and a supermultiplet $(S U(4))$, -spin $(S)$-isospin $(T))$ part

$$
\begin{aligned}
\text { Full cfp }= & {\left.\left.\left[\frac{\mathcal{N}_{\left[f_{n-1}\right]}}{\mathscr{N}_{\left[f_{n}\right]}}\right]^{1 / 2}\left\langle\left[f_{n-1}\right] \alpha_{n-1} L_{n-1} ;[1] \ell\right|\right\}\left[f_{n}\right] \alpha_{n} L_{n}\right\rangle } \\
& \left.\left.\times\left\langle\left[\tilde{f}_{n-1}\right] \beta_{n-1} S_{n-1} T_{n-1} ; \frac{1}{2} \frac{1}{2}\right|\right\}\left[\tilde{f}_{n}\right] \beta_{n} S_{n} T_{n}\right\rangle
\end{aligned}
$$

where $\mathscr{N}$ is the dimension of the irreducible representation of the permutation group on $n$ objects described by the Young pattern labels $\left[f_{n}\right]$. The contragredient representation describing the symmetry of the $n$-particle spin-isospin function is denoted by $\left[\tilde{f}_{n}\right]$. The labels $\alpha$ are short hand notation for all space quantum numbers other than $L ; \beta$ describes the supermultiplet quantum numbers other than $S, T$, when needed. For $1 p$ shell calculations the space part of the cfp is a reduced $S U$ (3) Wigner coefficient (in the $S U(3) \supset R(3)$ scheme). For $2 s-1 d$ (and higher) shells, the space part of the cfp can be factored into two parts; one involving the higher symmetries, and a second which is again a reduced $S U(3)$ Wigner coefficient (matrix element of a reduced Wigner operator).

Space part of $\mathrm{cfp}=$

$$
\begin{aligned}
& \left\langle\left[f_{n-1}\right] a_{n-1}\left(\lambda_{n-1} \mu_{n-1}\right) ;[1]\left(\lambda_{1} \mu_{1}\right) \|\left[f_{n}\right] a_{n}\left(\lambda_{n} \mu_{n}\right)\right\rangle\left\langle\left(\lambda_{n-1} \mu_{n-1}\right) K_{n-1} L_{n-1} ;\right. \\
& \left.\quad\left(\lambda_{1} \mu_{1}\right) \rho_{1} \|\left(\lambda_{n} \mu_{n}\right) K_{n} L_{n}\right\rangle .
\end{aligned}
$$

The $S U(3)$ irrep labels have been written in the Elliott notation $(\lambda \mu)=\left(m_{13}-m_{23}\right.$, $m_{23}-m_{33}$ ). In the $2 s-1 d$ shell the Young pattern labels $[f]$ characterize irreducible representations of $S U(6)$. Additional labels, $a$, are needed to fully characterize the $S U(6) \supset S U(3)$ part of the group chain in this case. The reduced $S U(3) \supset R(3)$ Wigner coefficients are characterized by $(\lambda \mu)$; the orbital angular momentum $\ell$; and an additional label $K$. (The difficult technical problems associated with this additional label have been discussed by several authors; see, e.g., J. D. Vergados [18].) 
The 27-plet operator arises as one of the most important $U(3)$ tensor operators making up the residual two-body interaction in the $1 p$ and $2 s-1 d$ shells. It is also one of the irreducible tensor pieces making up the most general one-body operator in the $2 s-1 d$ shell. The calculation of nuclear matrix elements will be illustrated for a one body operator in the $2 s-1 d$ shell. Let $\mathcal{O}$ be a one-body operator

$$
\mathcal{O}=\sum_{i=1}^{n} 0
$$

of definite irreducible tensor character characterized by $\left(\lambda_{0} \mu_{0}\right), L_{0}, M_{L_{0}},\left[\tilde{f}_{0}\right], S_{0}$, $M_{S_{0}}, T_{0}, M_{T_{0}}$. The one-particle matrix elements can be factored through a generalized Wigner-Eckart theorem

$$
\begin{aligned}
\left\langle\left(\lambda_{1} \mu_{1}\right) \ell{ }^{\prime} m_{\ell} m_{s}{ }^{\prime} m_{t}{ }^{\prime}|0|\left(\lambda_{1} \mu_{1}\right) \ell m_{\ell} m_{8} m_{t}\right\rangle \\
=\langle \\
\quad \\
\left.\quad \times\left\langle\lambda_{1} \mu_{1}\right)\|0\|\left(\lambda_{1} \mu_{1}\right)\right\rangle\left\langle\left(\lambda_{1} \mu_{1}\right) \ell ;\left(\lambda_{0} \mu_{0}\right) L_{0} M_{L_{0}} \mid \ell^{\prime} m_{\ell}{ }^{\prime}\right\rangle\left\langle\frac{1}{2} m_{s} S_{0} M_{S_{0}} \mid \frac{1}{2} m_{s}{ }^{\prime}\right\rangle\left\langle\frac{1}{2} m_{t} m_{t} T_{0} M_{T_{0}} \mid \frac{1}{2} m_{t}{ }^{\prime}\right\rangle,
\end{aligned}
$$

with $\left(\lambda_{1} \mu_{1}\right)=(20)$ in the $2 s-1 d$ shell. The matrix element of an operator $\mathcal{O}$ between $n$-nucleon states can be expressed by the cfp expansion

$$
\begin{gathered}
\left\langle\left[f^{\prime}\right] a^{\prime}\left(\lambda^{\prime} \mu^{\prime}\right) K^{\prime} L^{\prime} M_{L}{ }^{\prime} ; \beta^{\prime} S^{\prime} M_{S}{ }^{\prime} T^{\prime} M_{T}{ }^{\prime}|\mathcal{O}|[f] a(\lambda \mu) K L M_{L} ; \beta S M_{S} T M_{T}\right\rangle \\
=\sum_{\left[f_{n-1}\right]} \frac{\mathscr{N}_{\left[f_{n-1}\right]}}{\left[\mathcal{M}_{\left[f f^{\prime}\right.} \mathscr{N}_{\left[f^{\prime}\right]}\right]^{1 / 2}} \mathscr{F} \text { (space) } \mathscr{F} \text { (spin-isospin), }
\end{gathered}
$$

where

$\mathscr{F}$ (space)

$$
\begin{aligned}
= & \sum_{a_{n-1}\left(\lambda_{n-1} \mu_{n-1}\right)}\left\langle\left[f_{n-1}\right] a_{n-1}\left(\lambda_{n-1} \mu_{n-1}\right) ;(20) \|[f] a(\lambda \mu)\right\rangle\langle(20)\|0\|(20)\rangle \\
& \times\left\langle\left[f_{n-1}\right] a_{n-1}\left(\lambda_{n-1} \mu_{n-1}\right) ;(20) \|\left[f^{\prime}\right] a^{\prime}\left(\lambda^{\prime} \mu^{\prime}\right)\right\rangle \\
& \times\left\{\sum_{K_{n-1} L_{n-1} \ell \ell^{\prime}}\left\langle\left(\lambda_{n-1} \mu_{n-1}\right) K_{n-1} L_{n-1} ;(20) \ell \|(\lambda \mu) K L\right\rangle\left\langle(20) \ell ;\left(\lambda_{0} \mu_{0}\right) L_{0} \|(20) \ell^{\prime}\right\rangle\right. \\
& \times\left\langle\left(\lambda_{n-1} \mu_{n-1}\right) K_{n-1} L_{n-1} ;(20) \ell^{\prime} \|\left(\lambda^{\prime} \mu^{\prime}\right) K^{\prime} L^{\prime}\right\rangle \\
& \left.\times\left[\frac{(2 L+1)\left(2 \ell^{\prime}+1\right)}{\left(2 L_{n-1}+1\right)\left(2 L_{0}+1\right)}\right]^{1 / 2}(-1)^{L_{0}+L_{n-1} \ell^{\prime}-L} \times U\left(L \ell L^{\prime} \ell^{\prime} ; L_{n-1} L_{0}\right)\right\} \\
& \times\left\langle L M_{L} L_{0} M_{L_{0}} \mid L^{\prime} M_{L}^{\prime}\right\rangle .
\end{aligned}
$$


The corresponding spin-isospin part has been discussed in detail in Ref. [19]. By identifying the spin-isospin part of the cfp as a reduced supermultiplet $S U(4)$ Wigner coefficient it was possible to carry out the spin-isospin cfp sums and express $\mathscr{F}$ (spin-isospin) in terms of $S U(4)$ Racah coefficients and reduced $S U(4)$ matrix elements involving only final and initial state quantum numbers. In exact analogy with the techniques outlined in Ref. 19 it is possible to sum the $S U(3)$ part of $\mathscr{F}$ (space), enclosed in curly brackets in the cfp expansion (4.17). The result gives

$$
\begin{aligned}
\mathscr{F}(\text { space })= & \sum_{a_{n-1}\left(\lambda_{n-1} \mu_{n-1}\right)}\left\langle\left[f_{n-1}\right] a_{n-1}\left(\lambda_{n-1} \mu_{n-1}\right) ;(20) ![f] a(\lambda \mu)\right\rangle \\
& \times\left\langle\left[f_{n-1}\right] a_{n-1}\left(\lambda_{n-1} \mu_{n-1}\right) ;(20) \|\left[f^{\prime}\right] a^{\prime}\left(\lambda^{\prime} \mu^{\prime}\right) \backslash\langle(20)\|0\|(20)\rangle\right. \\
& \times\left\{\sum_{\gamma_{92}} \frac{U\left((\lambda \mu)(02)\left(\lambda^{\prime} \mu^{\prime}\right)(20) ;\left(\lambda_{n-1} \mu_{n-1}\right)--;\left(\lambda_{0} \mu_{0}\right)-\gamma_{22}\right)}{U\left((\lambda \mu)(02)(\lambda \mu)(20) ;\left(\lambda_{n-1} \mu_{n-1}\right)--;(00)--\right)}\right. \\
& \times \frac{\left\langle(\lambda \mu) K L ;\left(\lambda_{0} \mu_{0}\right) L_{0}\left(\gamma_{22}\right) \mid\left(\lambda^{\prime} \mu^{\prime}\right) K^{\prime} L^{\prime}\right\rangle !}{\left[\frac{1}{2}\left(\lambda_{0}+1\right)\left(\mu_{0}+1\right)\left(\lambda_{0}+\mu_{0}+2\right)\right]^{1 / 2}}\left\langle L M_{L} L_{0} M_{L_{0} !} ! L^{\prime} M_{L}^{\prime}\right\rangle
\end{aligned}
$$

i.e., sums over the $S U(3)$ subgroup-labels have been performed. [To simplify the notation we used the symbol "--" to indicate that the value of the correspondent $\gamma_{22}^{\prime}$ is not relevant since there is no multiplicity involved. The label $\gamma_{22}$ accounts for the multiplicity of $\left(\lambda_{0} \mu_{0}\right)$ which can be (22), (11) or $(0,0)$, i.e., the irreducible components of $(02) \otimes(20)$.] The reduced $S U(3) \supset R(3)$ Wigner coefficients appearing in the final result involve only initial and final state quantum numbers, but the resultant expression also involves $U(3)$ Racah or $U$-coefficients which describe the recoupling process for $(\lambda \mu) \times(02) \times(20) \rightarrow\left(\lambda^{\prime} \mu^{\prime}\right)$. The $U$-coefficient in the denominator, involving the identity representation $(00)$ is a seemingly complicated way of writing trivial $S U(3)$ dimensional and phase factors which arise from the symmetry relations needed to permute certain of the $S U(3)$ representations. By writing the result as a ratio of $U$-coefficients, however, the result is independent of the particular phase conventions chosen for the Wigner coefficients of the $S U(3) \supset R(3)$ scheme.

The Racah coefficients have the symmetry

$$
\begin{aligned}
& U\left((\mu \lambda)(02)\left(\mu^{\prime} \lambda^{\prime}\right)(20) ;\left(\mu^{\prime \prime} \lambda^{\prime \prime}\right)--;\left(\mu_{0} \lambda_{0}\right)-\gamma_{22}\right) \\
& \quad=(-1)^{\rho(\gamma)} U\left((\lambda \mu)(20)\left(\lambda^{\prime} \mu^{\prime}\right)(02) ;\left(\lambda^{\prime \prime} \mu^{\prime \prime}\right)-\cdots ;\left(\lambda_{0} \mu_{0}\right)-\gamma_{22}\right),
\end{aligned}
$$

where $\rho(\gamma)$ is defined in Eq. (3.3). The latter are a by-product of the present calculation. For $\left(\lambda_{0} \mu_{0}\right)=(22)$, if $\left(\lambda^{\prime} \mu^{\prime}\right)$ corresponds to one of the 12 outermost (single) points of the weight diagram of Fig. 1, the $U$-coefficients are unity. $U$-coefficients 
for the remaining $\left(\lambda^{\prime} \mu^{\prime}\right)$ are tabulated in Appendix I. The $S U(3)$ Racah coefficients which are a natural byproduct of the present caculation can therefore be used to simplify the calculation of nuclear shell model matrix elements.

\section{TABLES}

\section{Multiplicity Three Cases}

These cases comprise the operators with operator pattern $(\gamma)=\left({ }_{4}{ }^{2}{ }_{0}\right),\left({ }_{3}{ }^{2}{ }_{1}\right)$, $\left(2_{2}{ }_{2}\right)$ and their parities are $+1,-1,+1$, respectively. The matrix elements for the operators

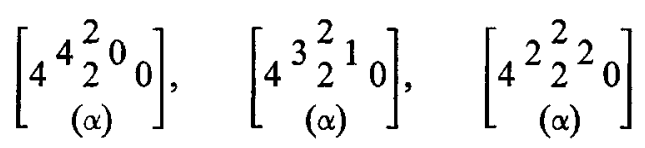

are given in Tables I to IV, as follows: The common "pattern factor" $f(\alpha)$ part is given in Table I; the $F(\alpha, \gamma)$ parts are given in Tables II, III, IV. The normalization factors are

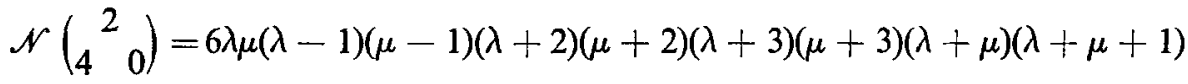

$$
\begin{aligned}
& \times(\lambda+\mu-3)(\lambda+\mu \mid-4)(g-4)(g-9), \\
& \mathscr{N}\left(\begin{array}{c}
2 \\
3
\end{array}\right)=\frac{2}{5} \lambda \mu(\lambda+2)(\mu+2)(\lambda+\mu+1)(\lambda+\mu+3) g(g-9), \\
& \mathscr{N}\left(\begin{array}{cc}
2 & 2 \\
2 & 2
\end{array}\right)=\frac{3}{10} g(g-4) \text {. }
\end{aligned}
$$

Here $g$ is the second-order Casimir invariant of $S U(3)$ as defined in Appendix II.

[It is of interest to note that the zeros of the invariants $\mathscr{N}$ explicitly give the null spaces associated with the operators. That is, $\mathscr{N}\left({ }_{2}{ }_{2}{ }_{2}\right)$ vanishes for $g=0$ or $g=4$, i.e., for the irreps [000], [100], [110]; $\mathscr{N}\left({ }_{3}{ }^{2}{ }_{1}\right)$ vanishes for the particular irreps [000] and [210] as well as for all, irreps of the form [p00] or $[p p 0] ; \mathcal{N}\left({ }_{4}{ }^{2}{ }_{0}\right)$ vanishes for all irreps of the form [p00], [p10], [pp0], $[p p-10]$.

It is an interesting, and significant, fact that these null spaces are simply ordered; that is, the null space of $\left({ }_{4}^{2}{ }_{0}\right)$ includes that of $\left({ }_{3}^{2}{ }^{2}\right)$ which, in turn, includes that of $\left({ }_{2}^{2}{ }_{2}\right)$.] 


\section{Multiplicity Two Cases}

These cases comprise the operators with operator patterns:

$$
\begin{array}{r}
(\gamma)=\left(\begin{array}{ll}
3 \\
4 & 0
\end{array}\right),\left(\begin{array}{ll}
3 \\
3 & 1
\end{array}\right) \\
\left(\begin{array}{ll}
3 \\
4 & 1
\end{array}\right),\left(\begin{array}{ll}
3 & 2
\end{array}\right) \\
\left(\begin{array}{ll}
1 & 0 \\
4 & 0
\end{array}\right),\left(\begin{array}{ll}
1 & 1
\end{array}\right) \\
\left(\begin{array}{ll}
2 & 0 \\
3 & 0
\end{array}\right),\left(\begin{array}{ll}
2 & 1 \\
2 & 1
\end{array}\right) \\
\left(\begin{array}{ll}
2 & 1 \\
4 & 1
\end{array}\right),\left(\begin{array}{ll}
2 & 2 \\
3 & 2
\end{array}\right) \\
\left(\begin{array}{ll}
3 & 1 \\
3 & 0
\end{array}\right),\left(\begin{array}{ll}
1 & 1
\end{array}\right) .
\end{array}
$$

The matrix elements for the operators

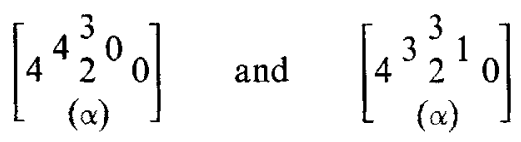

are given in Tables V, VI, and VII. The common $f(\alpha)$ part is given in Table V. The $F(\alpha, \gamma)$ parts are given in Tables VI and VII.

The normalization factors are

$$
\begin{aligned}
\mathscr{N}\left(\begin{array}{cc}
3 & \\
4 & 0
\end{array}\right)= & 2\left[2(\mu-1)(\mu+4)+\lambda(2 \mu+13)+3 \lambda^{2}\right] \lambda(\lambda+1)(\lambda+2) \\
& \times(\lambda+4)(\mu-1)(\mu+1)(\mu+2)(\lambda+\mu+1)(\lambda+\mu+2) \\
& \times(\lambda+\mu+4), \\
\mathscr{N}\left(\begin{array}{ll}
3 & \\
3 & 1
\end{array}\right)= & \frac{2}{5}(\lambda+1)(\lambda+2)(\mu+1)(\lambda+\mu+2)[2(\mu-1)(\mu+4) \\
& \left.+\lambda(2 \mu+13)+3 \lambda^{2}\right] .
\end{aligned}
$$


The other ten cases can be obtained from the listed ones using symmetry 3 together with Table XI.

\section{Multiplicity One Cases}

These cases comprise the operators with the operator labels:

$$
\begin{aligned}
& (\gamma)=\left(\begin{array}{ll}
1 & \\
2 & 0
\end{array}\right),\left(\begin{array}{ll}
4 & \\
4 & 1
\end{array}\right),\left(\begin{array}{ll}
1 & \\
4 & 1
\end{array}\right), \\
& \left(\begin{array}{cc}
3 & \\
4 & 2
\end{array}\right),\left(\begin{array}{cc}
3 \\
3 & 0
\end{array}\right),\left(\begin{array}{cc}
0 \\
3 & 0
\end{array}\right), \\
& \left(\begin{array}{cc}
0 \\
4 & 0
\end{array}\right),\left(\begin{array}{cc}
2 & 2 \\
4 & 2
\end{array}\right),\left(\begin{array}{cc}
2 & 0 \\
2 & 0
\end{array}\right),\left(\begin{array}{cc}
4 & 2
\end{array}\right),\left(\begin{array}{cc}
0 \\
2 & 0
\end{array}\right),\left(\begin{array}{cc}
4 & 0 \\
4 & 0
\end{array}\right) .
\end{aligned}
$$

The matrix elements for the operators

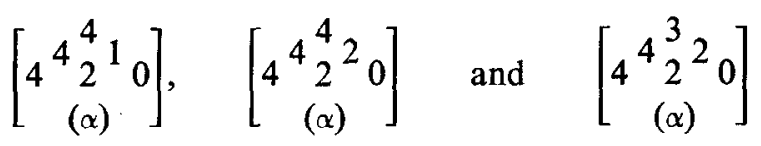

are given in Tables VIII, IX, X. Since these operators have a simple structure in these tables we list all three parts together $(f, F$, and $\mathscr{N})$, that is the complete expression for the matrix elements is given in the tables.

The matrix elements for the other nine operators can be obtained from the listed ones using symmetry 3 together with Table XI.

\section{How to Use the Tables}

To use the tables, one proceeds in the following way:

(1) Determine the multiplicity (3,2 or 1) of the operator and whether or not it is listed (see Section 5, above). If it is a listed operator, the appropriate table and the normalization $\mathscr{N}$ are given in the text (Section 5, above). One finds the two parts $f$ and $F$ in the designated tables; the complete operator is defined in Eq. (3.1) from $f, F$, and $\mathscr{N}$.

(2) If it is not a listed operator, we must use symmetry 3 of Section 3. The $f$, $F$, and $\mathscr{N}$ parts of the listed operator are then transformed by this symmetry into (the parts of) the operator one seeks. 


\section{Examples}

(1) Evaluation of the matrix element of

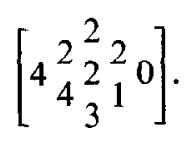

Since this is a multiplicity three operator, it is completely tabulated. We use Tables I and II to find its parts $f$ and $F$. The normalization factor $\left.\mathcal{N}_{(2}{ }_{2}{ }_{2}\right)$ is given by Eq. (5.3). Using Eq. (3.1) we have

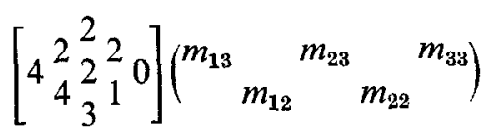

$$
\begin{aligned}
& =\frac{2}{3}\left[\frac{5(\lambda-p)(\mu+p+2)(p+1)(2 \Lambda)(2 \Lambda+3)}{(2 \Lambda+2) g(g-4)}\right]^{1 / 2} .
\end{aligned}
$$

(2) Evaluation of the matrix element of

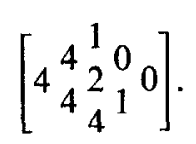

This is a multiplicity two operator and is not in the tables. However, a look at Table XI shows us that its matrix element is related to the matrix element of

$$
\left[4{ }_{4}^{4}{ }_{4}^{3}{ }_{1}^{0} 0\right]
$$

(which is in the tables) through symmetry 3 , i.e.,

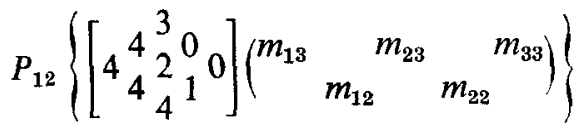

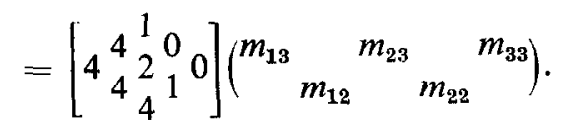


Now we use Tables V and VII and the normalization factor $\mathcal{N}\left({ }_{\mathbf{q}}{ }^{3}{ }_{0}\right)$ given by Eq. (5.4) to get

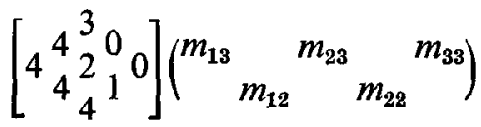

$$
\begin{aligned}
& =+\left[\frac{\left(p_{12}-p_{23}+1\right)\left(p_{22}-p_{33}\right)\left(p_{13}-p_{12}-1\right)\left(p_{13}-p_{22}\right)\left(p_{13}-p_{22}+1\right)\left(p_{12}-p_{23}+2\right)}{\left(p_{12}-p_{22}+1\right)\left(p_{12}-p_{22}+2\right)\left(p_{12}-p_{22}+3\right)\left[2(\mu-1)(\mu+4)+\lambda(2 \mu+13)+3 \lambda^{2}\right]}\right. \\
& \left.\times \frac{\left(p_{12}-p_{23}+3\right)\left(p_{12}-p_{33}+1\right)\left(p_{12}-p_{33}+2\right)}{\lambda(\lambda+1)(\lambda+2)(\lambda+4)(\mu-1)(\mu+1)(\mu+2)(\lambda+\mu+1)(\lambda+\mu+2)(\lambda+\mu+4)}\right]^{1 / 2} \\
& \times \frac{1}{3}\left\{(\epsilon+6 \Lambda)\left[2(\mu-1)(\mu+4)+(2 \mu+13) \lambda+3 \lambda^{2}\right]\right. \\
& \left.+4(\mu-1)(\mu+4)(\mu+3)+\left(3 \mu^{2}-\mu+28\right) \lambda-4(\mu+5) \lambda^{2}-6 \lambda^{3}\right\} \text {. }
\end{aligned}
$$

We now apply to this matrix element the transformation $P_{12}$

$$
p_{13} \leftrightarrow p_{23} \text { \{or equivalently }\left(\begin{array}{l}
\lambda \rightarrow-\lambda-2 \\
\mu \rightarrow \lambda+\mu+1
\end{array}\right. \text { ) }
$$

and finally get

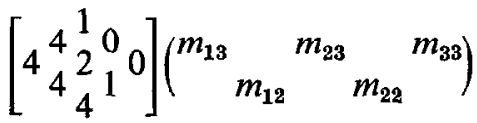

$$
\begin{aligned}
& =\left[\frac{\left[\begin{array}{c}
\left(p_{13}-p_{12}-1\right)\left(p_{22}-p_{33}\right)\left(p_{12}-p_{23}+1\right)\left(p_{23}-p_{22}\right)\left(p_{23}-p_{22}+1\right) \\
\times\left(p_{13}-p_{12}-2\right)\left(p_{13}-p_{12}-3\right)\left(p_{12}-p_{33}+1\right)\left(p_{12}-p_{33}+2\right.
\end{array}\right]}{\left[\begin{array}{c}
\left(p_{12}-p_{22}+1\right)\left(p_{12}-p_{22}+2\right)\left(p_{12}-p_{22}+3\right)[2(\lambda+\mu)(\lambda+\mu+5) \\
-(\lambda+2)(2 \lambda+2 \mu+15)+3(\lambda+2) \\
\times+(\lambda+2)(\lambda+1) \lambda(\lambda-2) \\
\times(\lambda+\mu)(\lambda+\mu+2)(\lambda+\mu+3) \mu(\mu+1)(\mu+3)
\end{array}\right]}\right]^{1 / 2} \\
& \times \frac{1}{3}\left\{(\epsilon+6 \Lambda)\left[2(\lambda+\mu)(\lambda+\mu+5)-(2 \lambda+2 \mu+15)(\lambda+2)+3(\lambda+2)^{2}\right]\right. \\
& +4(\lambda+\mu)(\lambda+\mu+4)(\lambda+\mu+5)-(\lambda+2)\left(3 \lambda^{2}+6 \lambda \mu+5 \lambda+3 \mu^{2}+5 \mu+30\right) \\
& \left.-4(\lambda+\mu+6)(\lambda+2)^{2}+6(\lambda+2)^{3}\right\} \text {. }
\end{aligned}
$$

In order to facilitate the use of the tables we could tabulate explicitly all $(27)^{2}=729$ matrix elements of the [4 20$]$ operator. But taking into account that probably the most useful of these operators will be those with multiplicity three, we decided for brevity to list only these completely, and rely on symmetry 3 to shorten the tabulation of the others. Symmetry 2 can be used to shorten the entries even further, but this is a difficult symmetry to use and requires an additional tabulation of the phase factors $\epsilon$. 


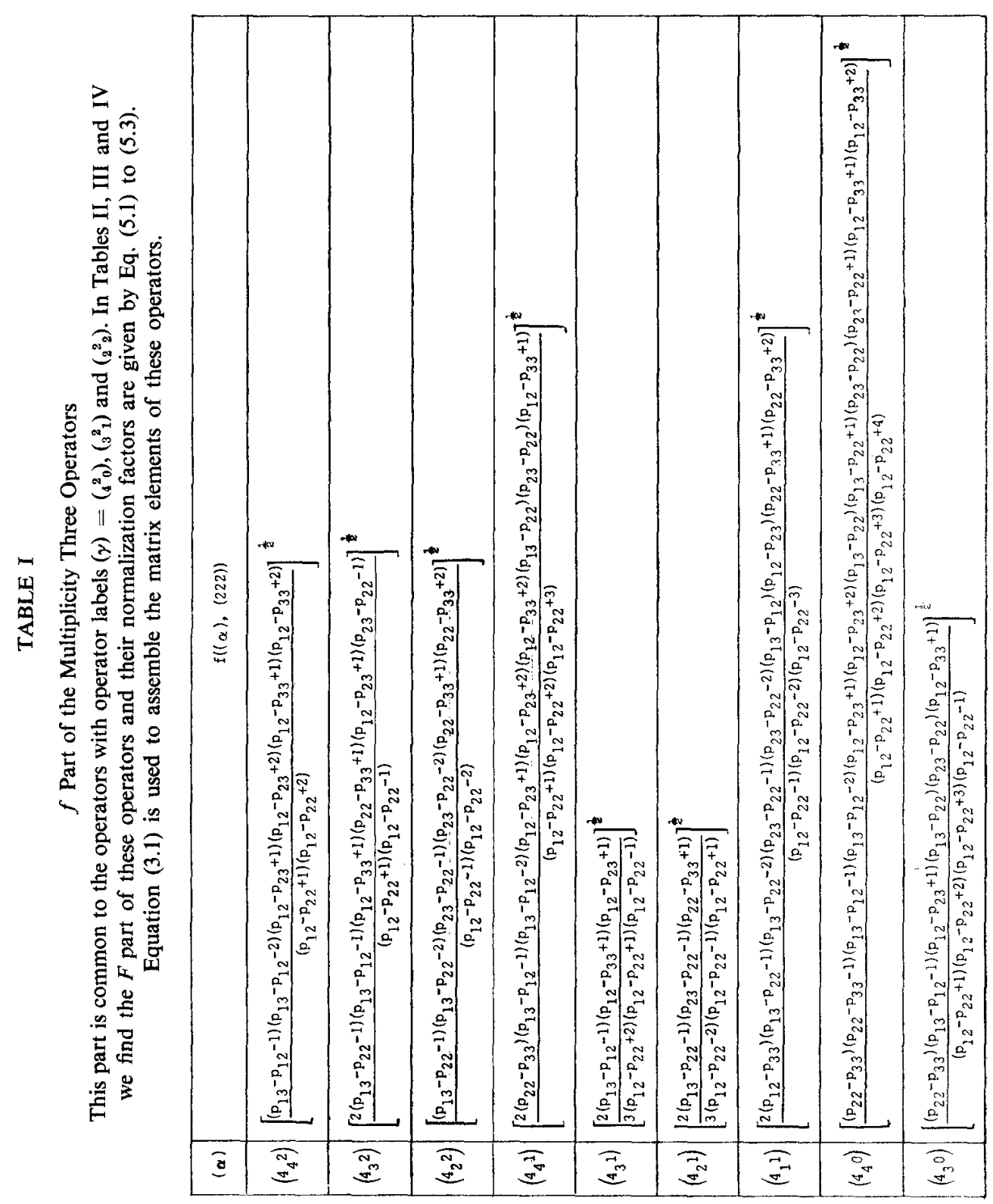




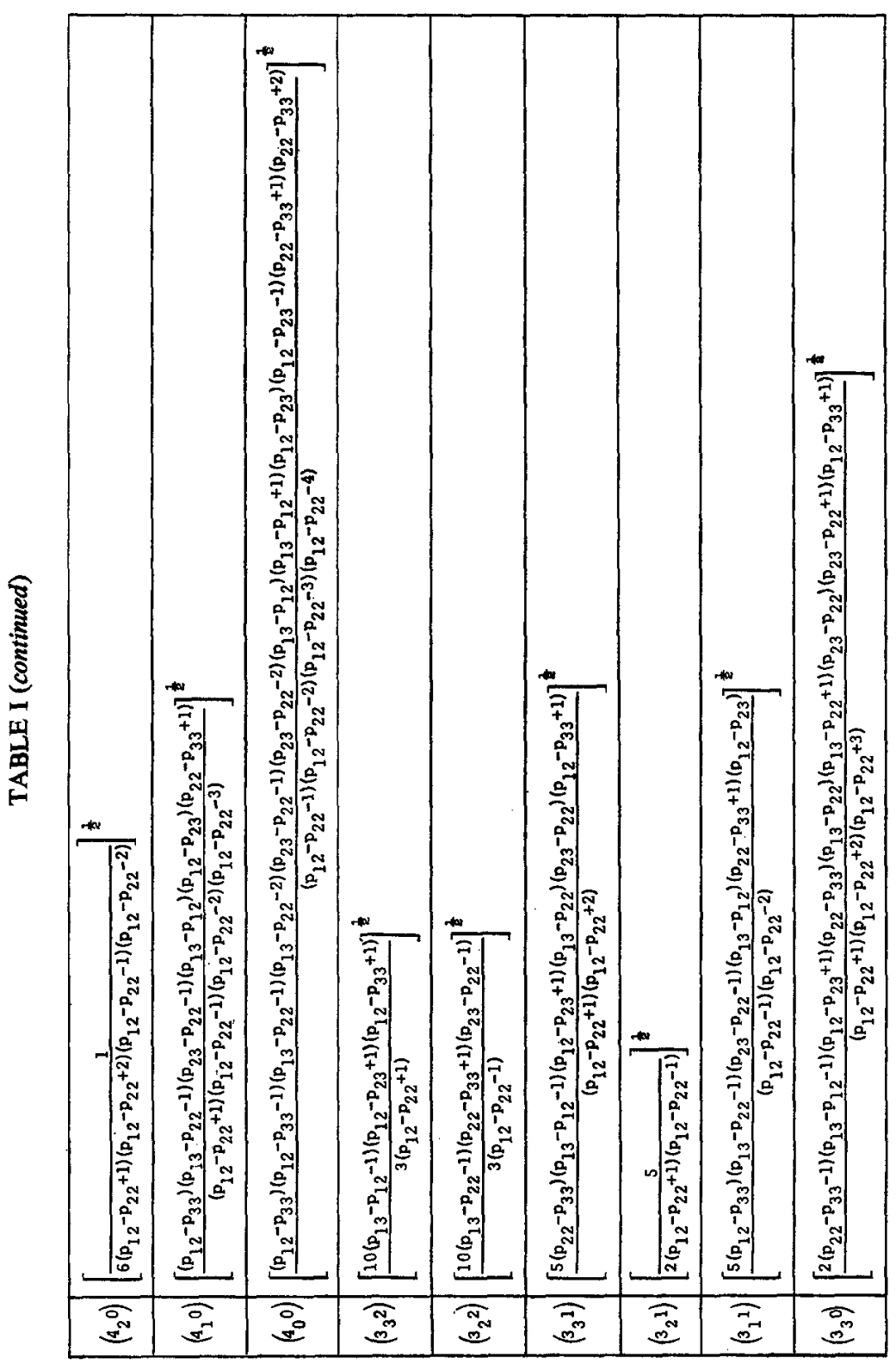




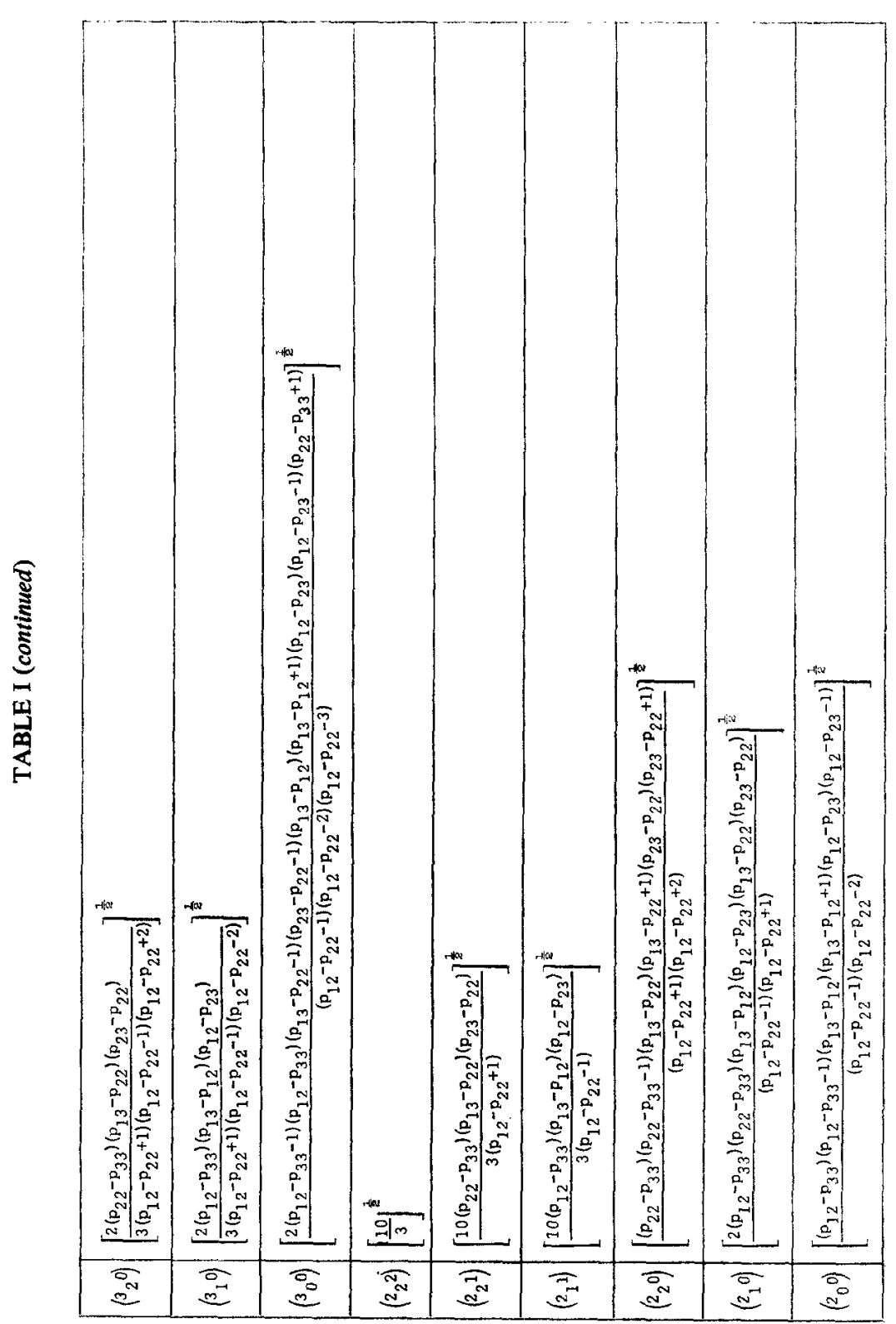


TABLE II

F Part for the Multiplicity Three Operator with Operator Labels $(\gamma)=\left({ }_{2}^{2}\right)$

Its $f$ part is given in Table I and its normalization factor is given by Eq. (5.3).

Equation (3.1) is used to assemble the matrix elements of the operator.

\begin{tabular}{|c|c|}
\hline$(\alpha)$ & $F(\alpha, 2,2,2)$ \\
\hline$\left(4_{4}^{4}\right)$ & 1 \\
\hline$\left(4_{3}{ }^{2}\right)$ & 3 \\
\hline$\left(4_{2}{ }^{2}\right)$ & 1 \\
\hline$\left(4_{4}{ }^{1}\right)$ & 0 \\
\hline$\left({ }^{4}{ }_{3} 1\right)$ & $(2 \Lambda)(2 \Lambda+3)$ \\
\hline$\left({ }_{4}^{4}{ }^{2}\right)$ & $(2 \Lambda-1)(2 \Lambda+2)$ \\
\hline$\left({ }_{4}^{4} 1^{1}\right)$ & 0 \\
\hline$\left(4_{4},\right)$ & 0 \\
\hline$\left(4{ }_{3} 0\right)$ & 0 \\
\hline
\end{tabular}

\begin{tabular}{|l|l|}
\hline$(\alpha)$ & $F(\alpha, 2,2,2)$ \\
\hline$\left(4_{2}{ }^{0}\right)$ & $(2 \Lambda-1)(2 \Lambda)(2 \Lambda+2)(2 \Lambda+3)$ \\
\hline$\left(4_{1} 0\right)$ & 0 \\
\hline$\left(4{ }^{0}\right)$ & 0 \\
\hline$\left(3_{3}^{2}\right)$ & $\frac{1}{10}(3 \epsilon-2 \Lambda-6)$ \\
\hline$\left(\beta_{2}^{2}\right)$ & $\frac{1}{10}(3 \epsilon+2 \Lambda-4)$ \\
\hline$\left(3_{3}\right)$ & $-\frac{2}{5}$ \\
\hline$\left(\beta_{2} 1\right)$ & $\frac{1}{270}\left[\epsilon^{3}+324 \epsilon \Lambda(\Lambda+1)-12 \epsilon(g+3)+8 \Gamma\right]$ \\
\hline$\left.\left(\beta_{1}\right)^{1}\right)$ & $\frac{2}{5}$ \\
\hline$\left(\beta_{3} 0\right)$ & 0 \\
\hline
\end{tabular}

\begin{tabular}{|l|l|}
\hline$(\alpha)$ & $F(\alpha, 2,2,2)$ \\
\hline$(3,0)$ & $-(2 \Lambda)(2 \Lambda+3)$ \\
\hline$(3,0)$ & $(2 \Lambda-1)(2 \Lambda+2)$ \\
\hline$(300)$ & 0 \\
\hline$\left(22^{2}\right)$ & $\frac{1}{10}\left[c^{2}+4 \Lambda(\Lambda+1)-g\right]$ \\
\hline$(2,1)$ & $\frac{-1}{10}(3 \epsilon+2 \Lambda+6)$ \\
\hline$\left(21^{1}\right)$ & $\frac{1}{10}(3 \epsilon-2 \Lambda+4)$ \\
\hline$(2,0)$ & 1 \\
\hline$(2,0)$ & -1 \\
\hline$(200)$ & 1 \\
\hline
\end{tabular}




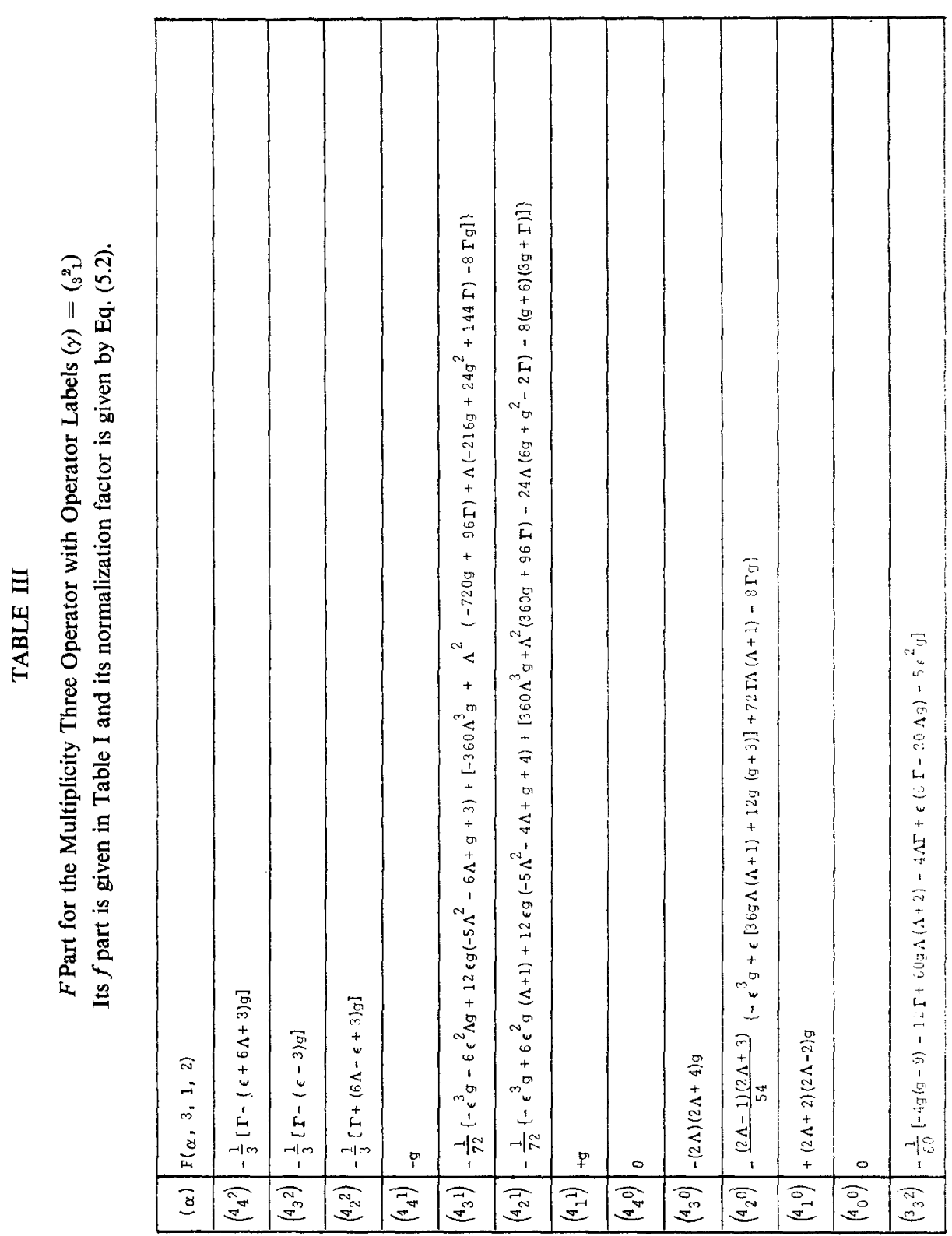




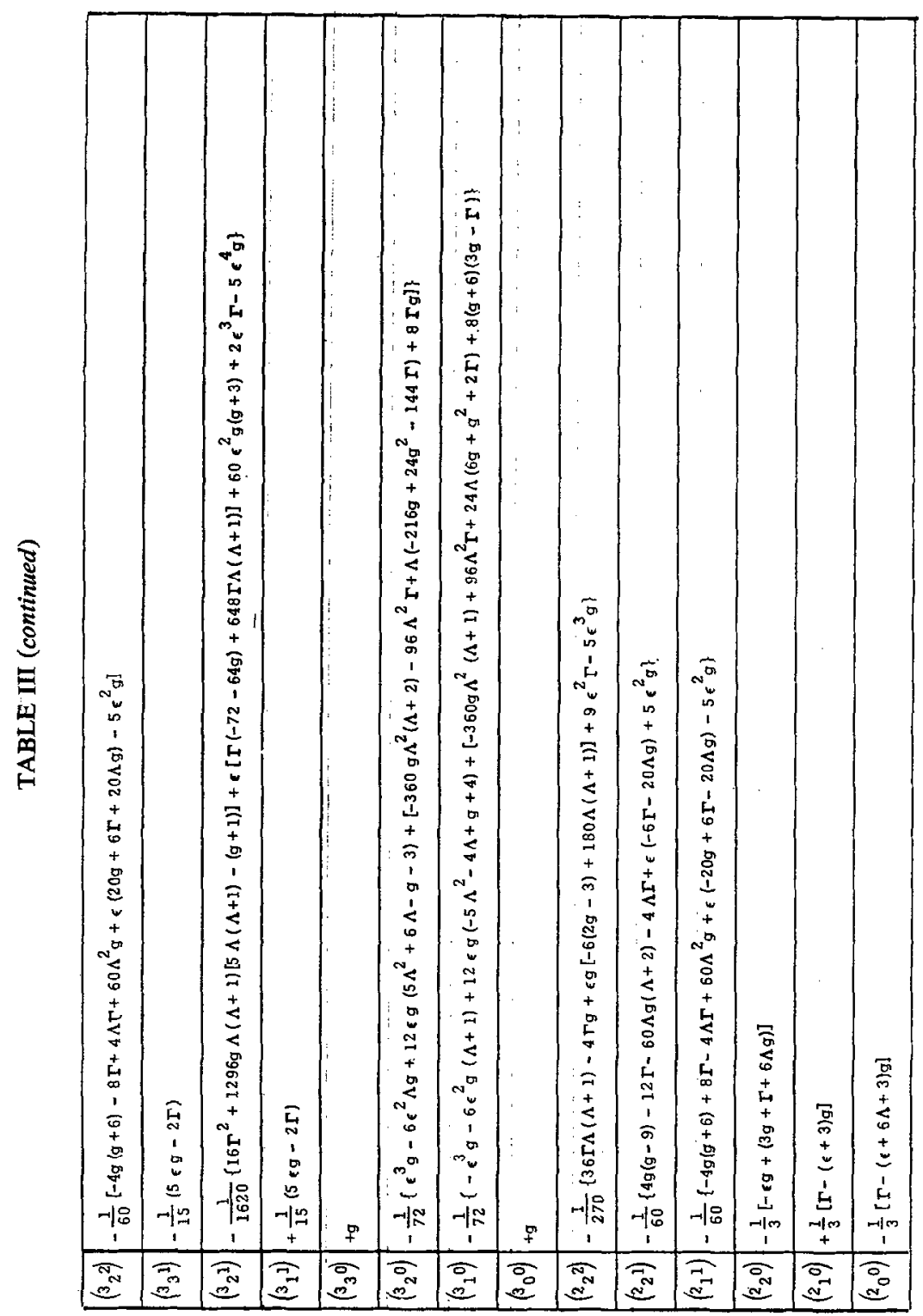




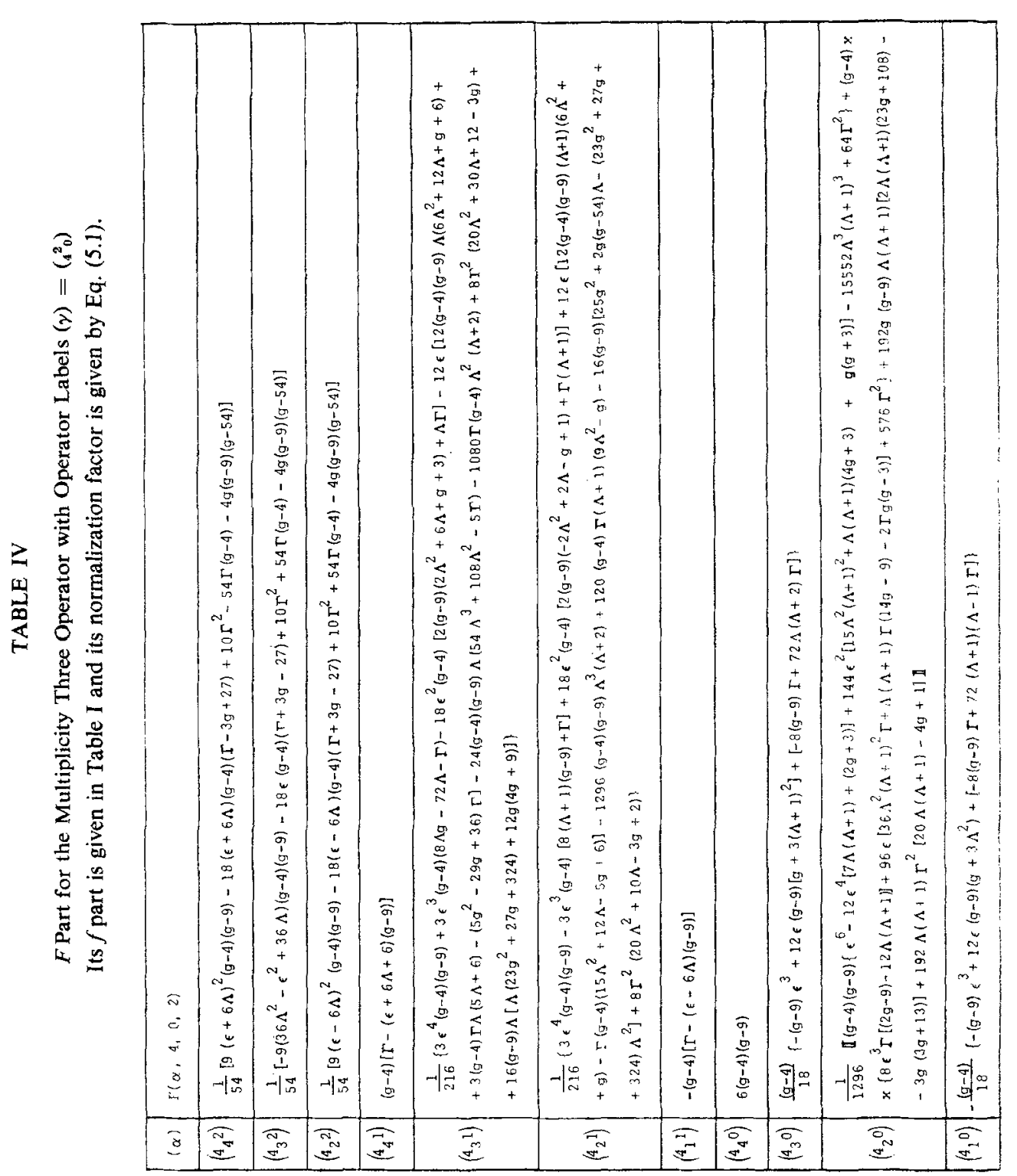




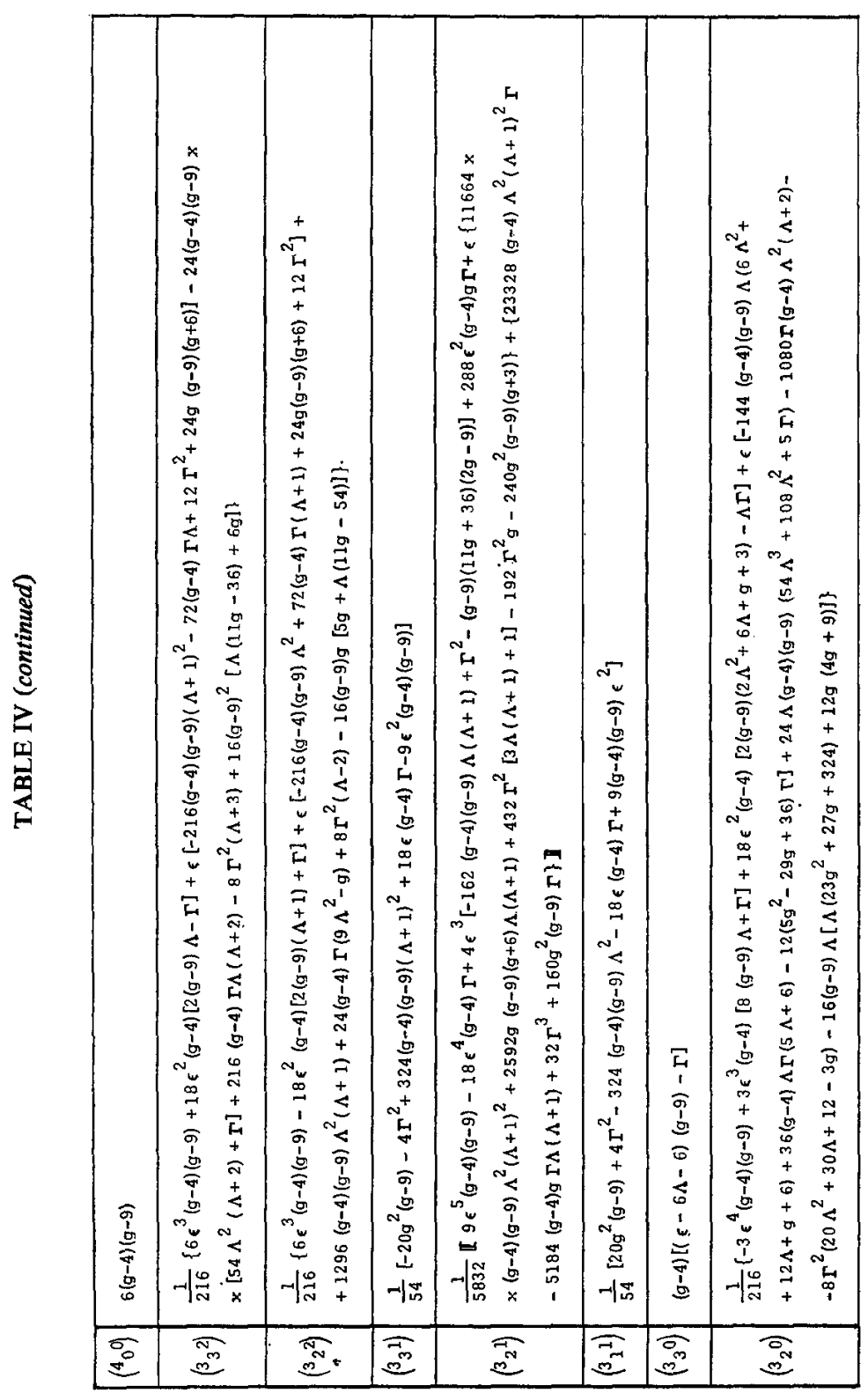




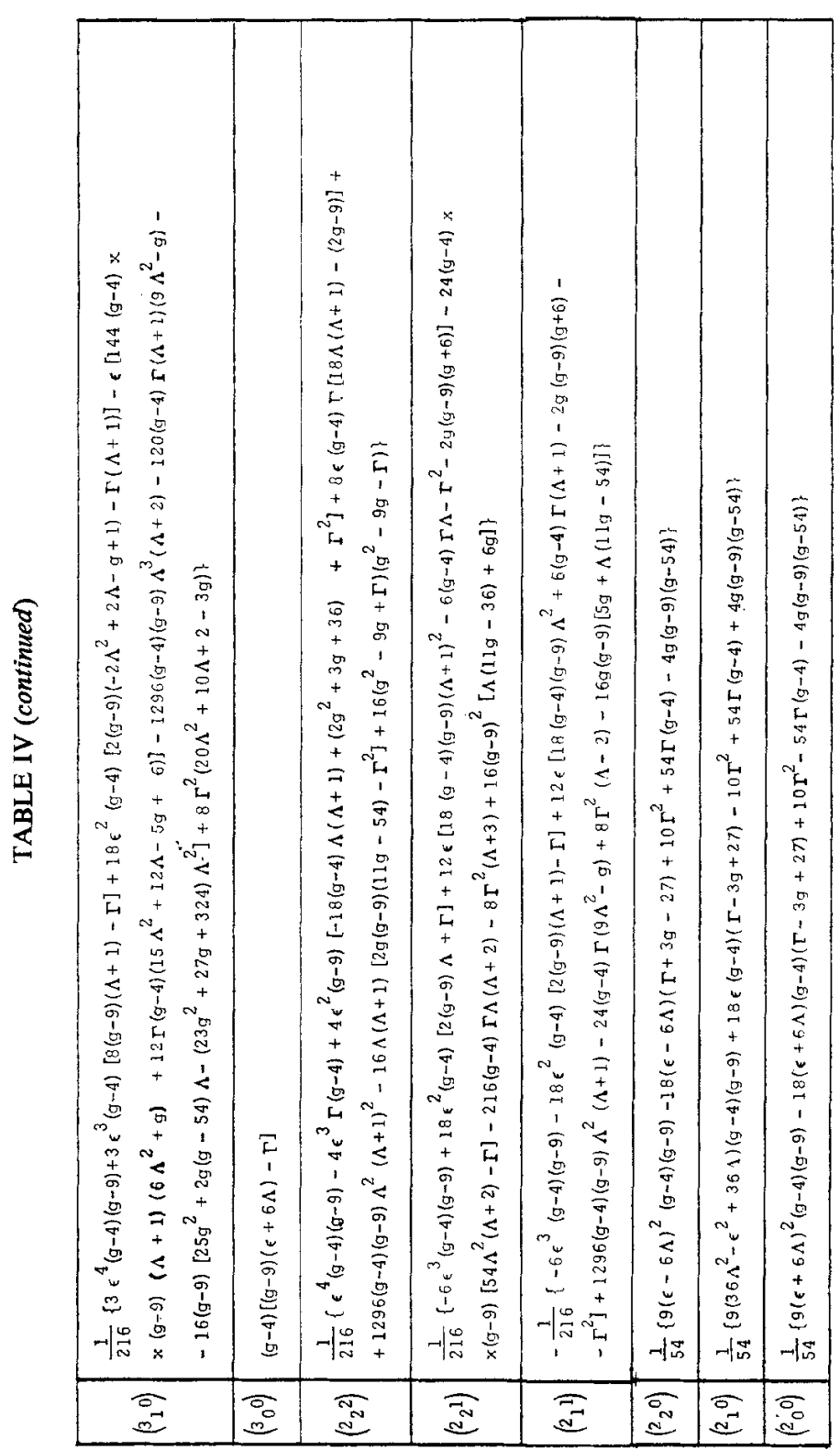




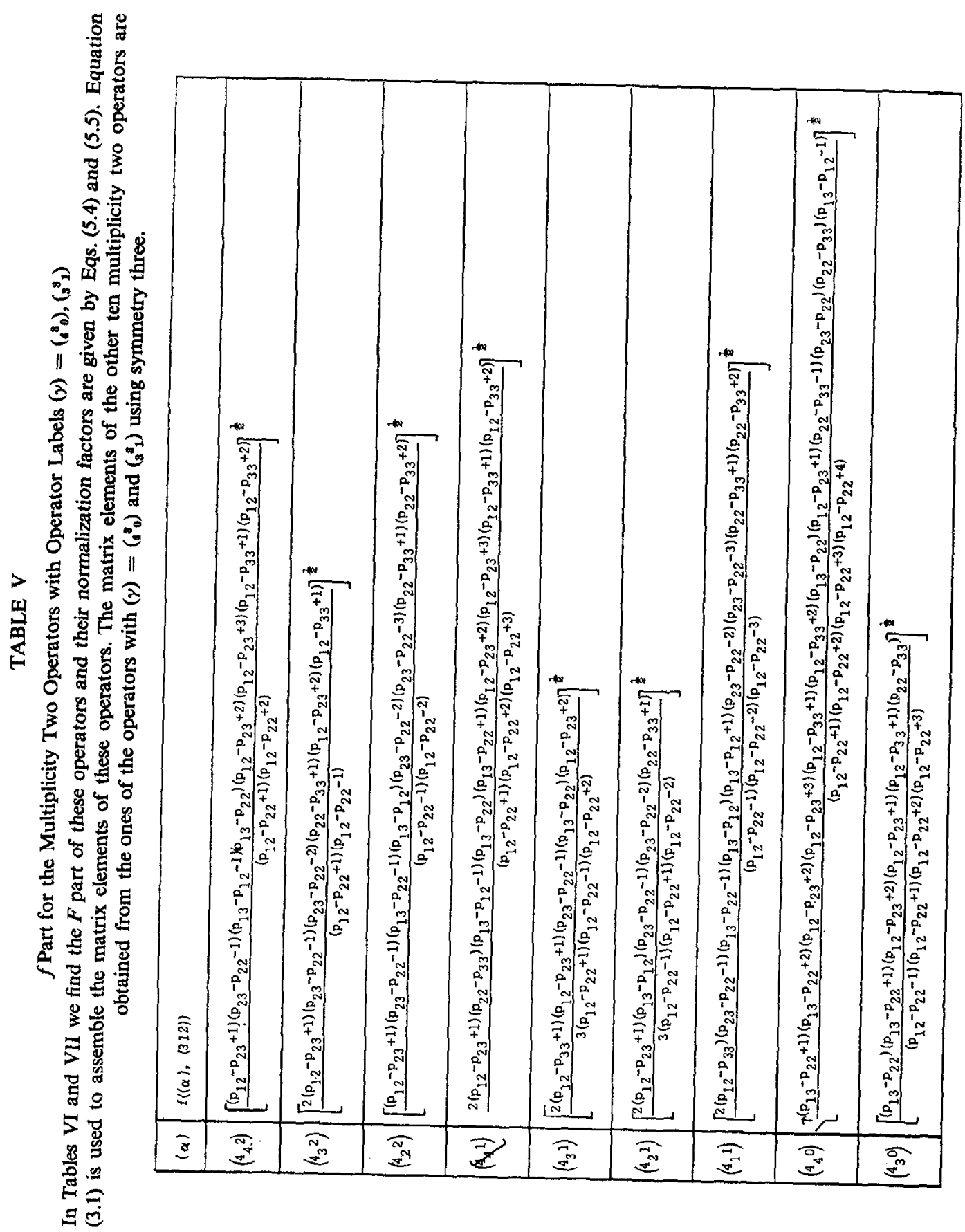




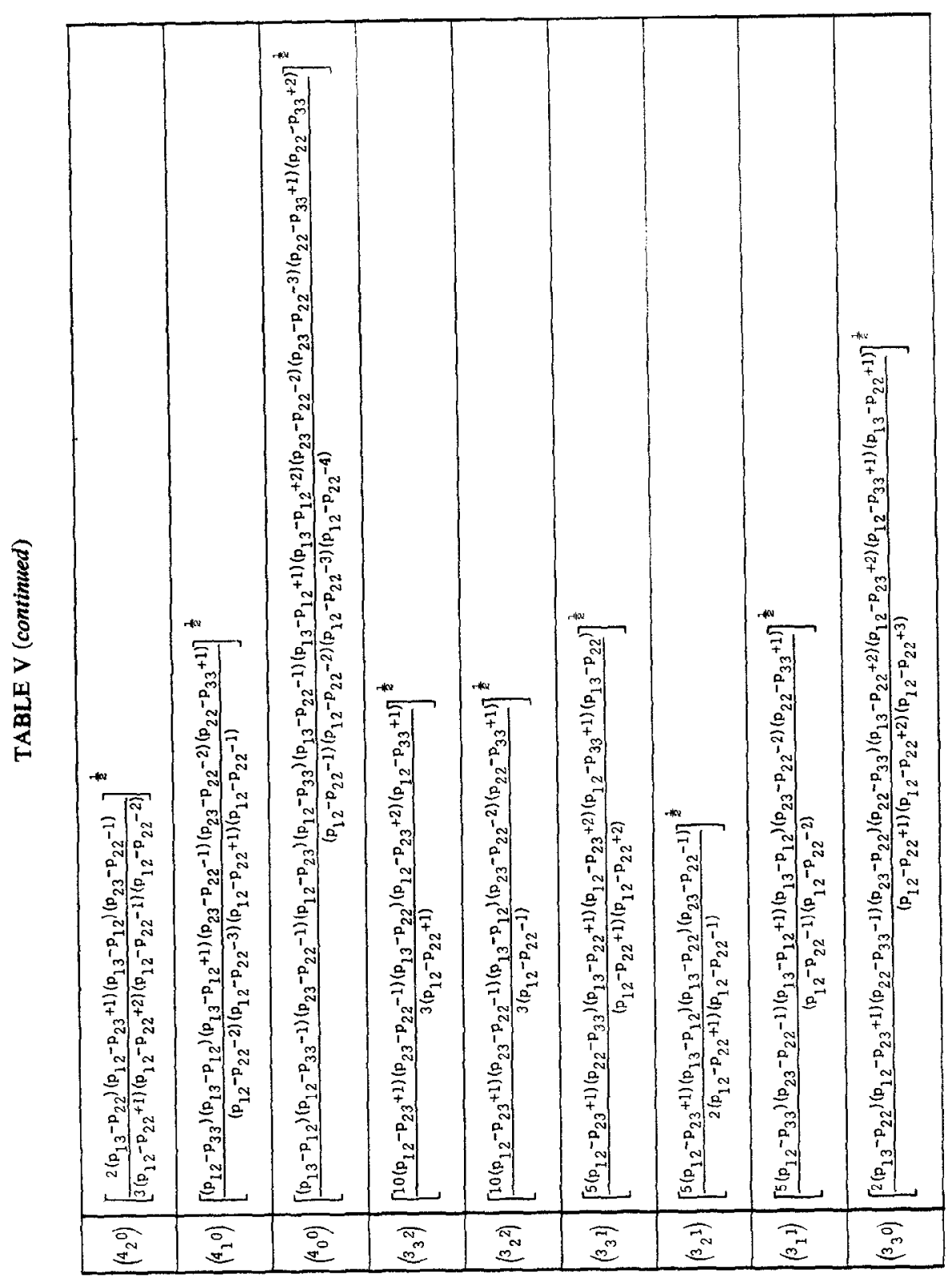




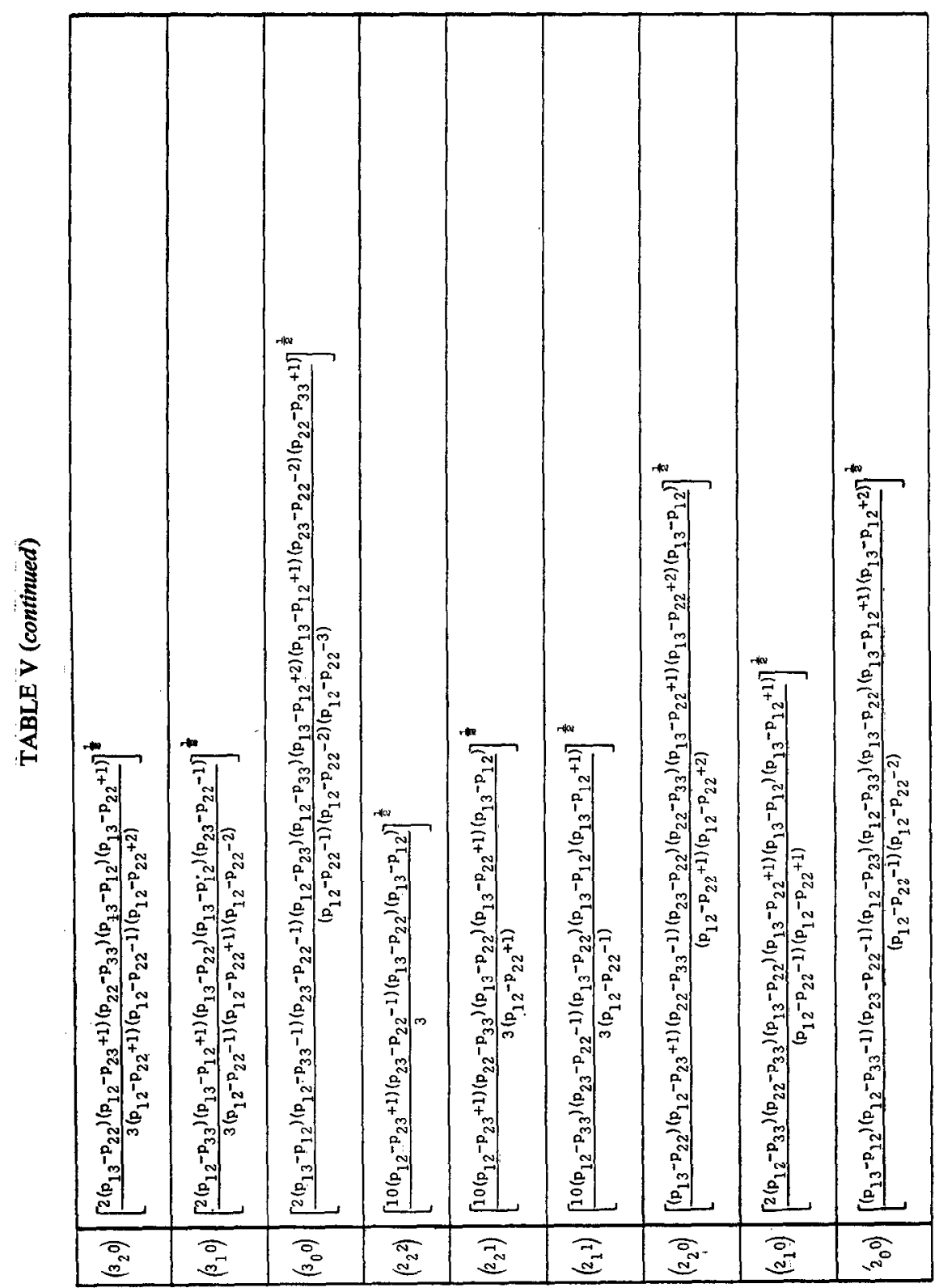




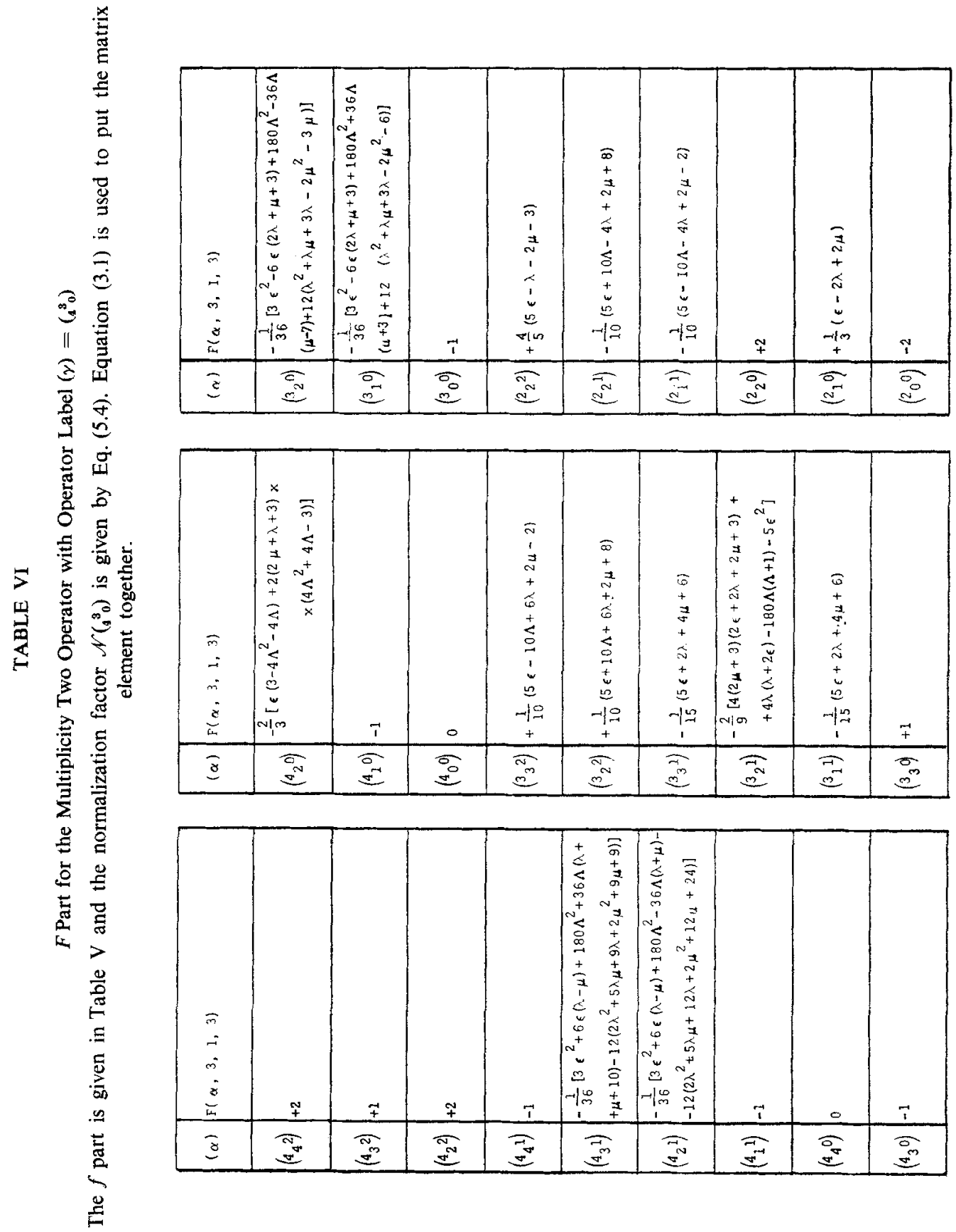




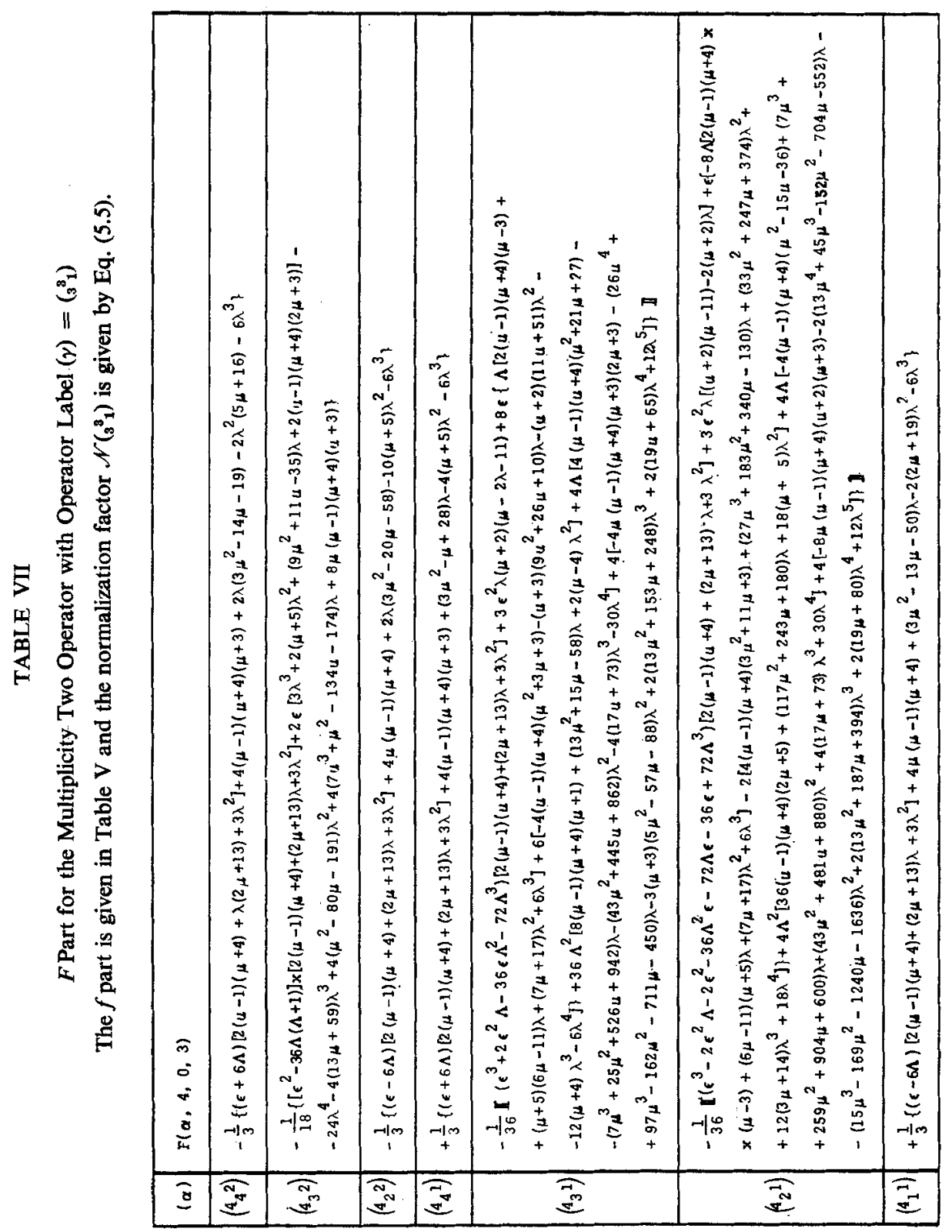




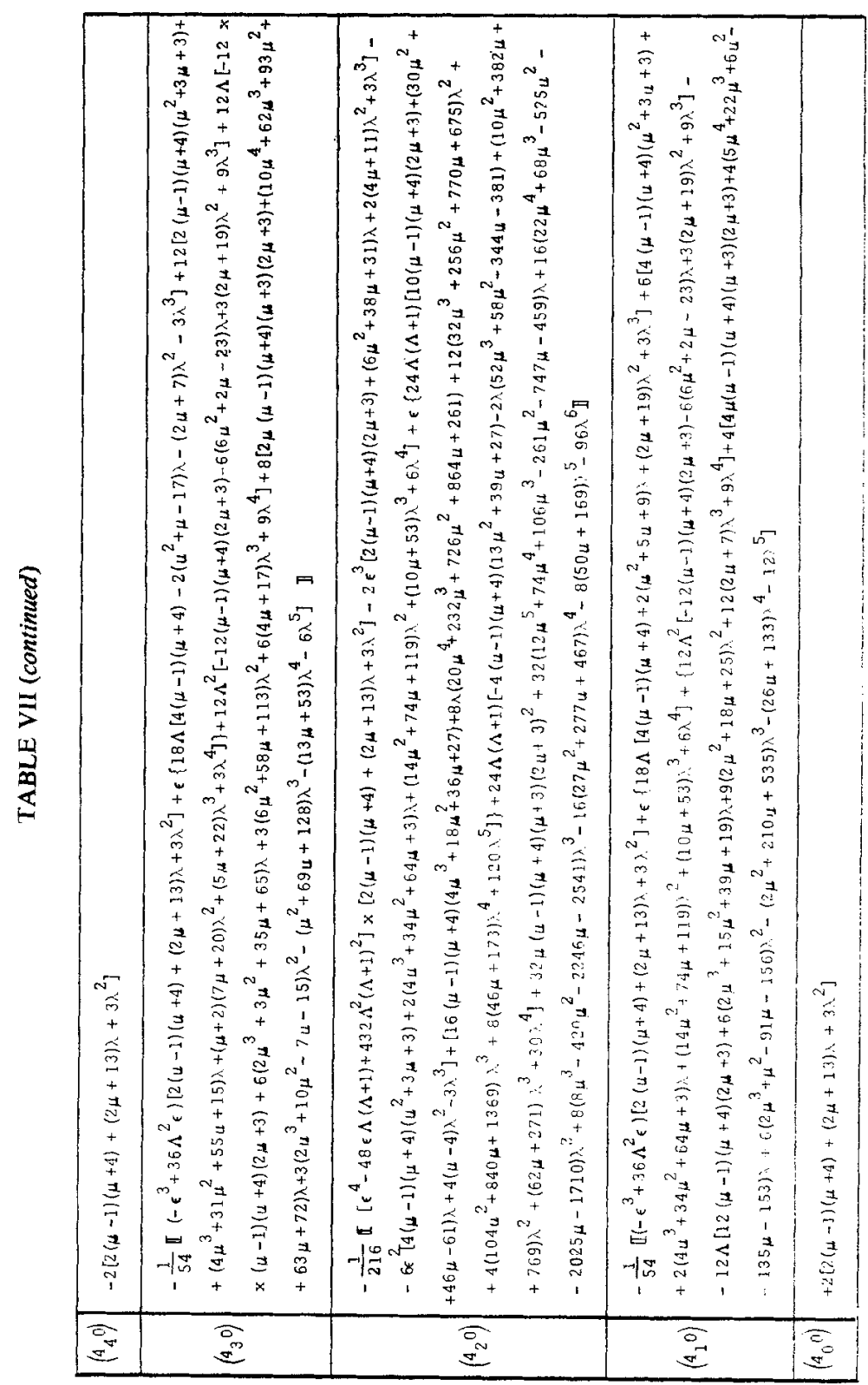




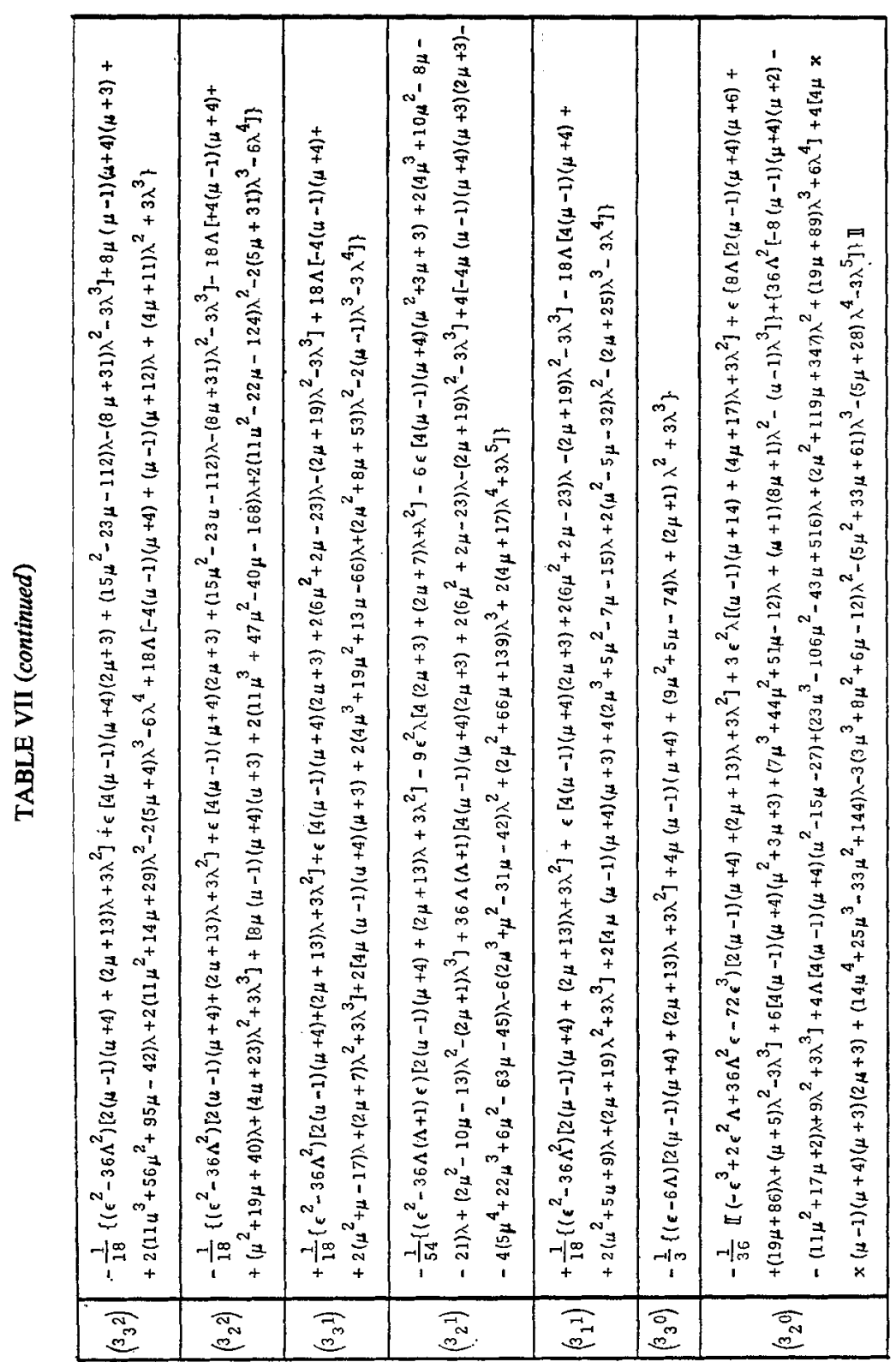




\begin{tabular}{|c|c|c|c|c|c|c|c|}
\hline 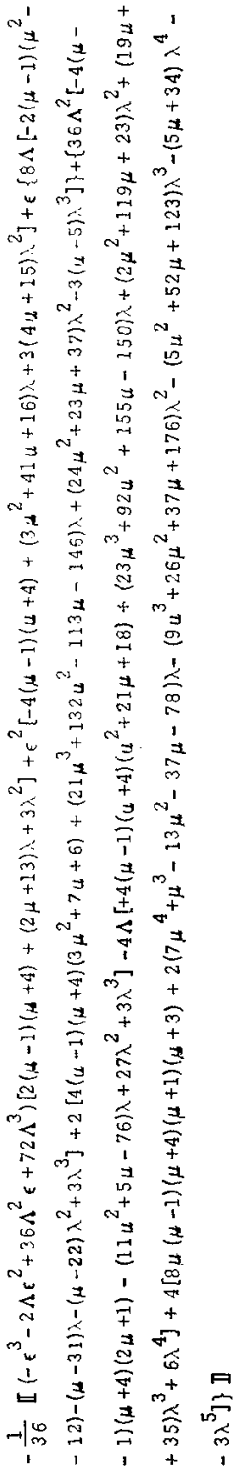 & 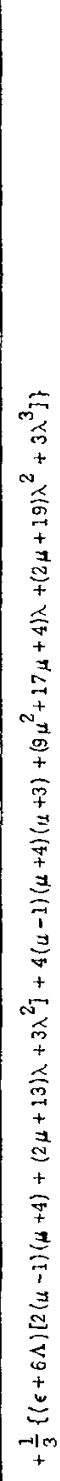 & 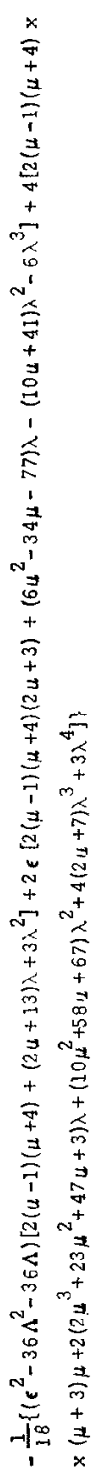 & 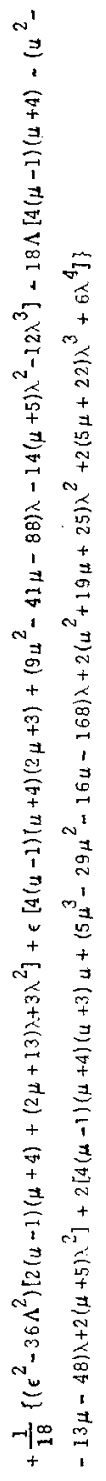 & 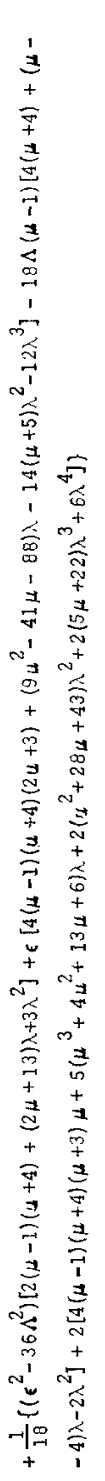 & 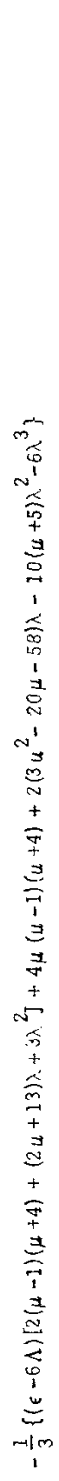 & 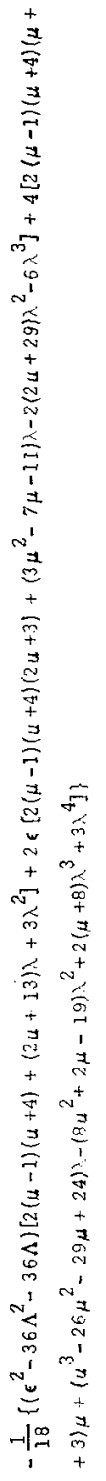 & 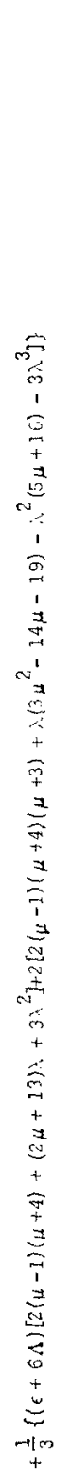 \\
\hline $\bar{D}$ & 응 & $\bar{N}$ & 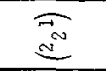 & & $\tilde{i}^{N}$ & I & $\stackrel{\partial}{a}$ \\
\hline
\end{tabular}




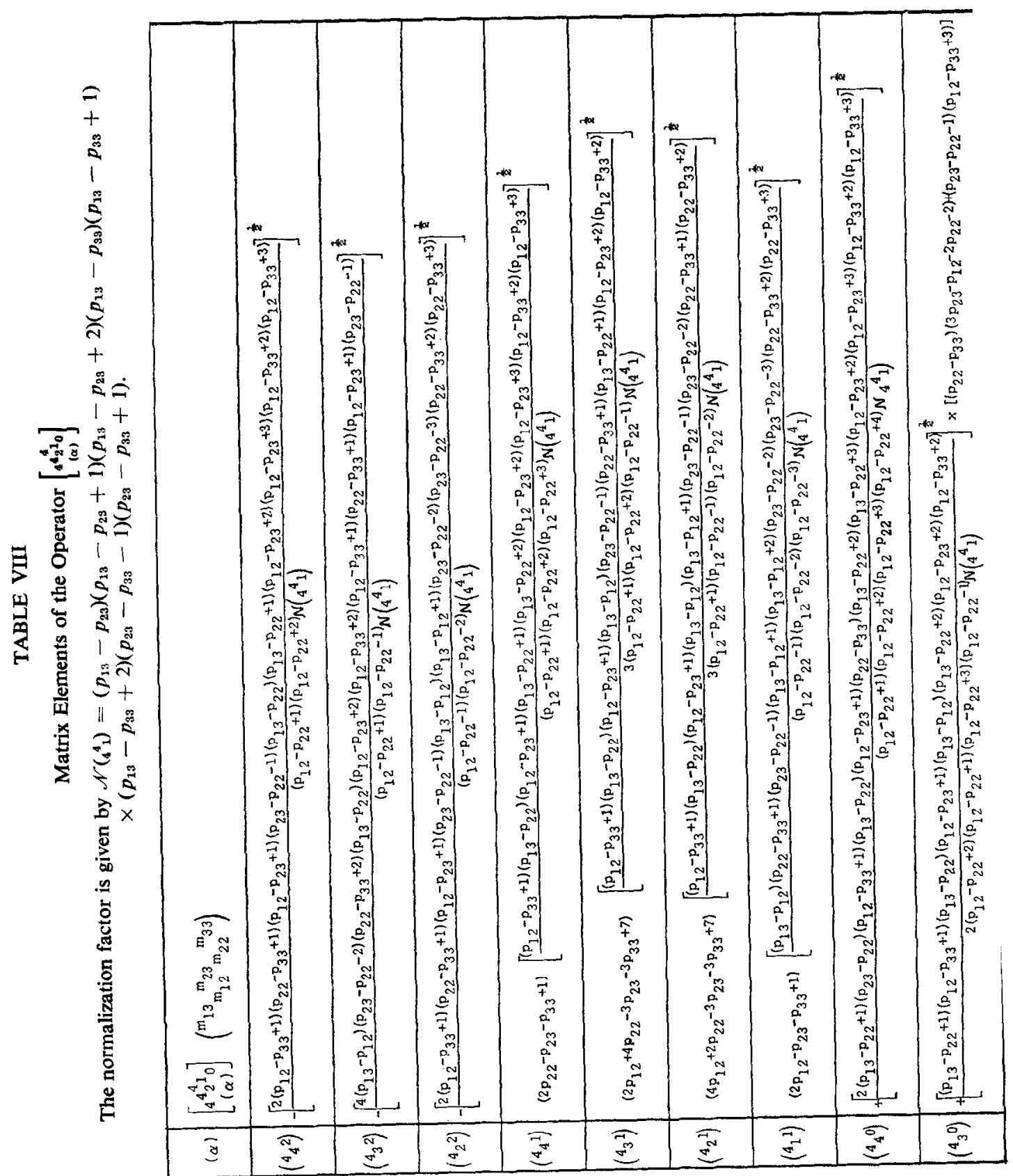




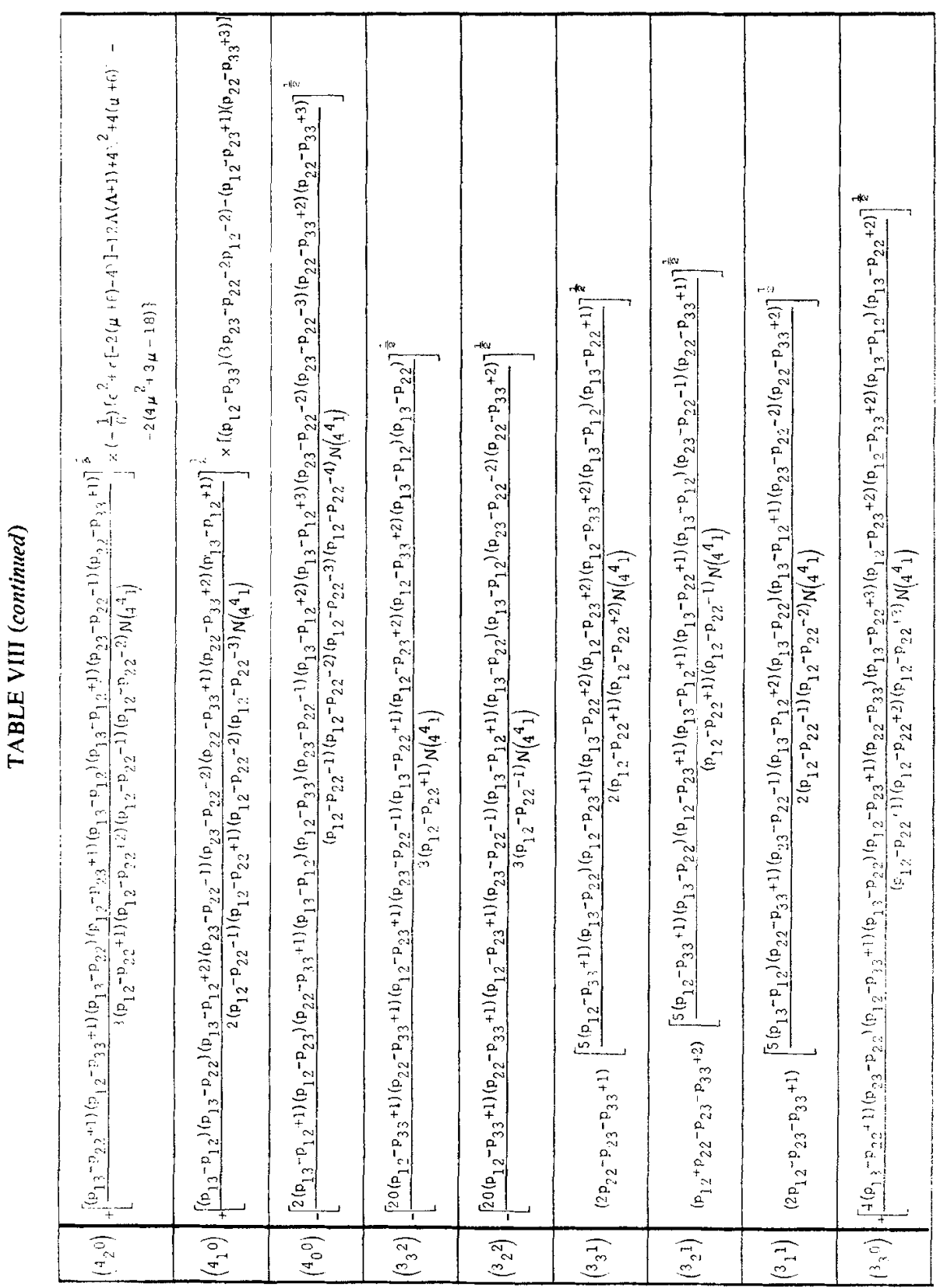




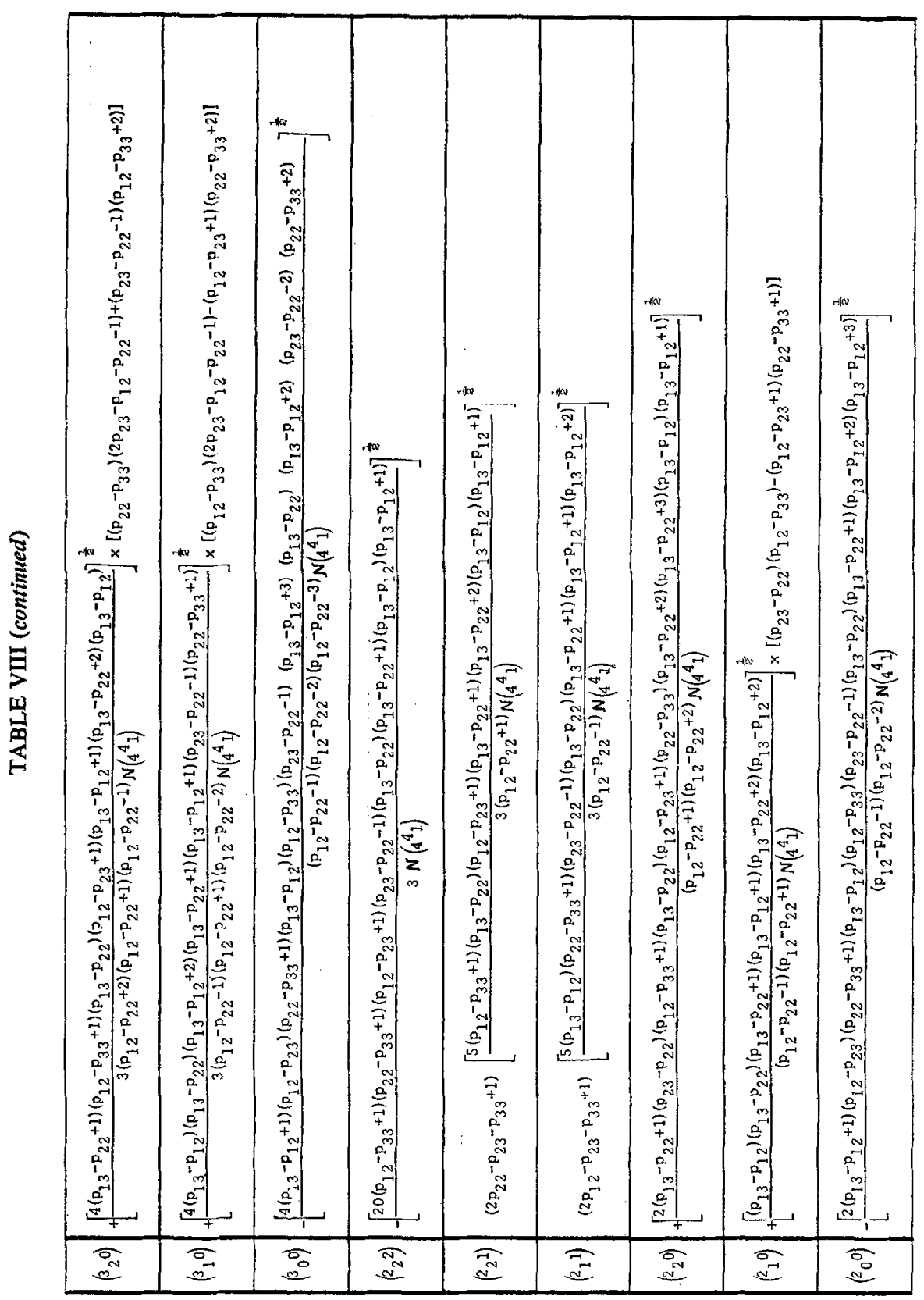




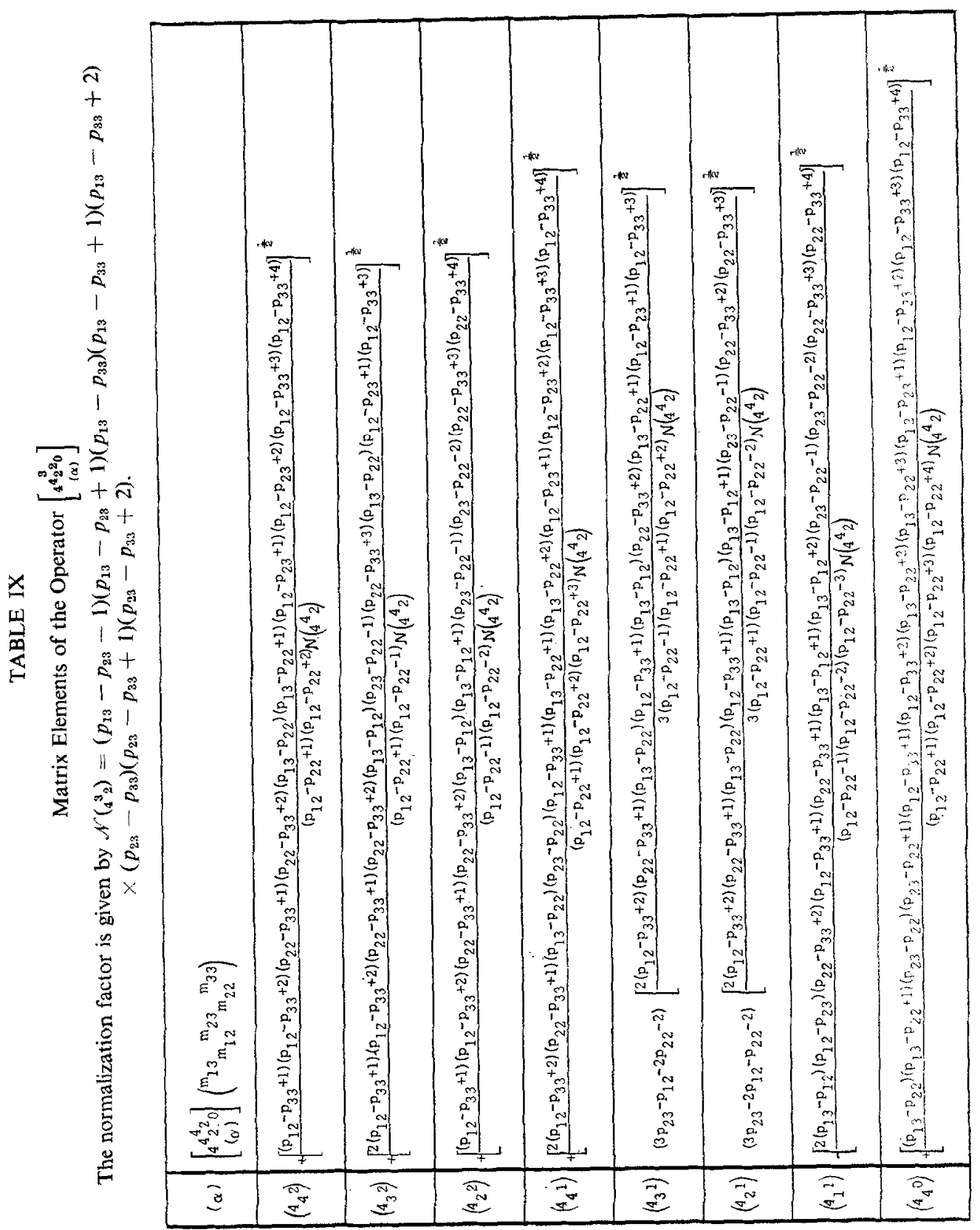




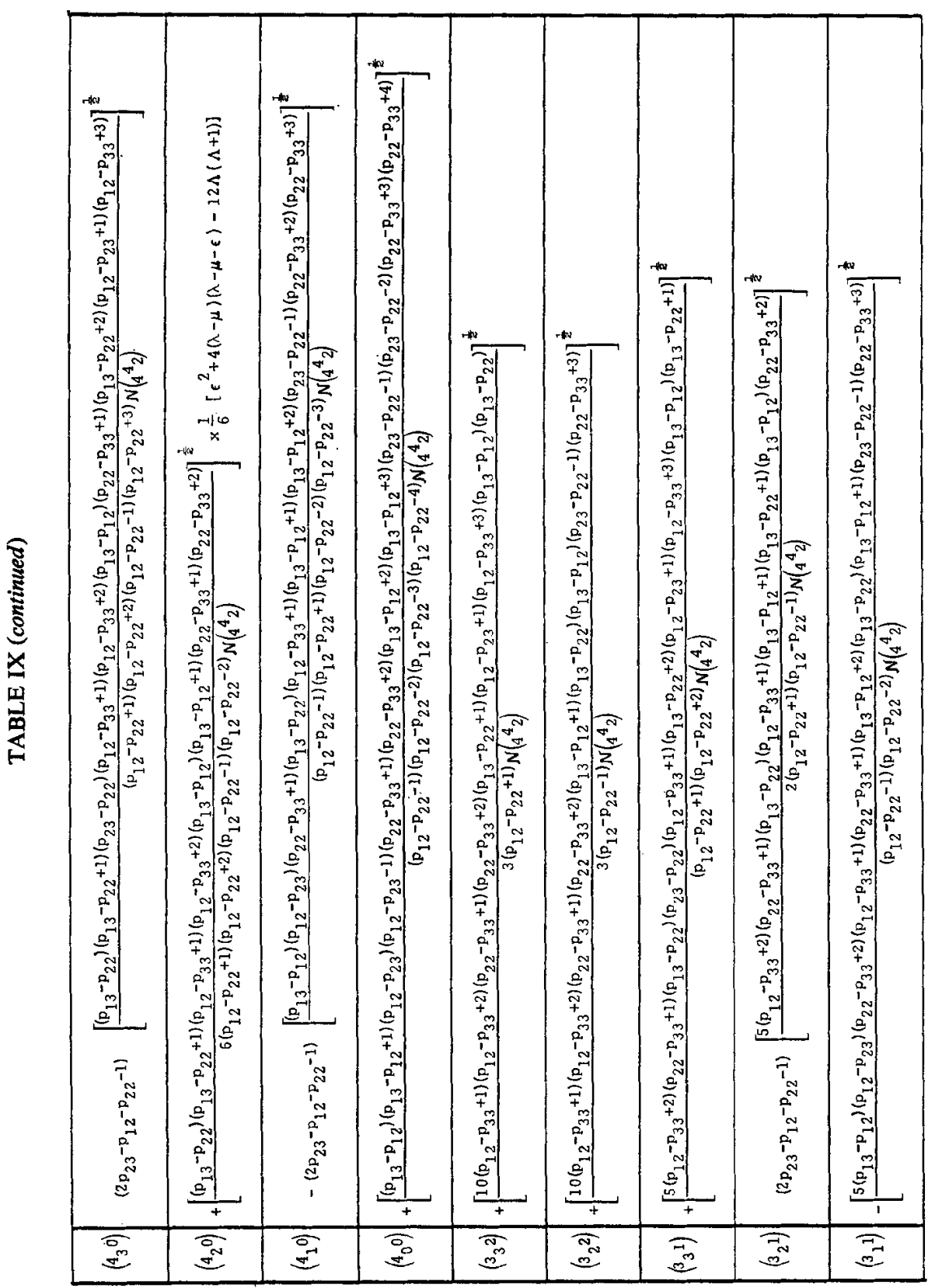




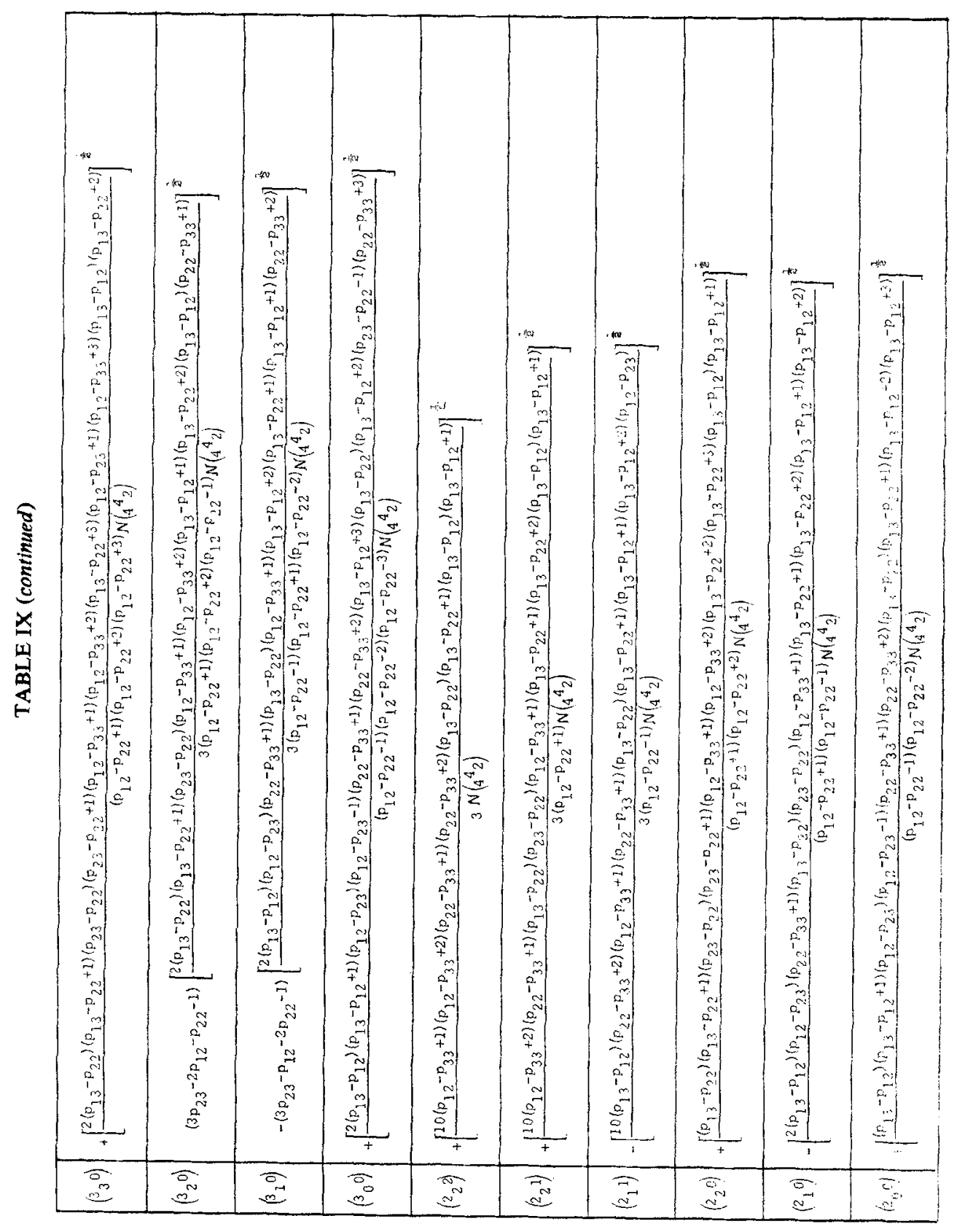




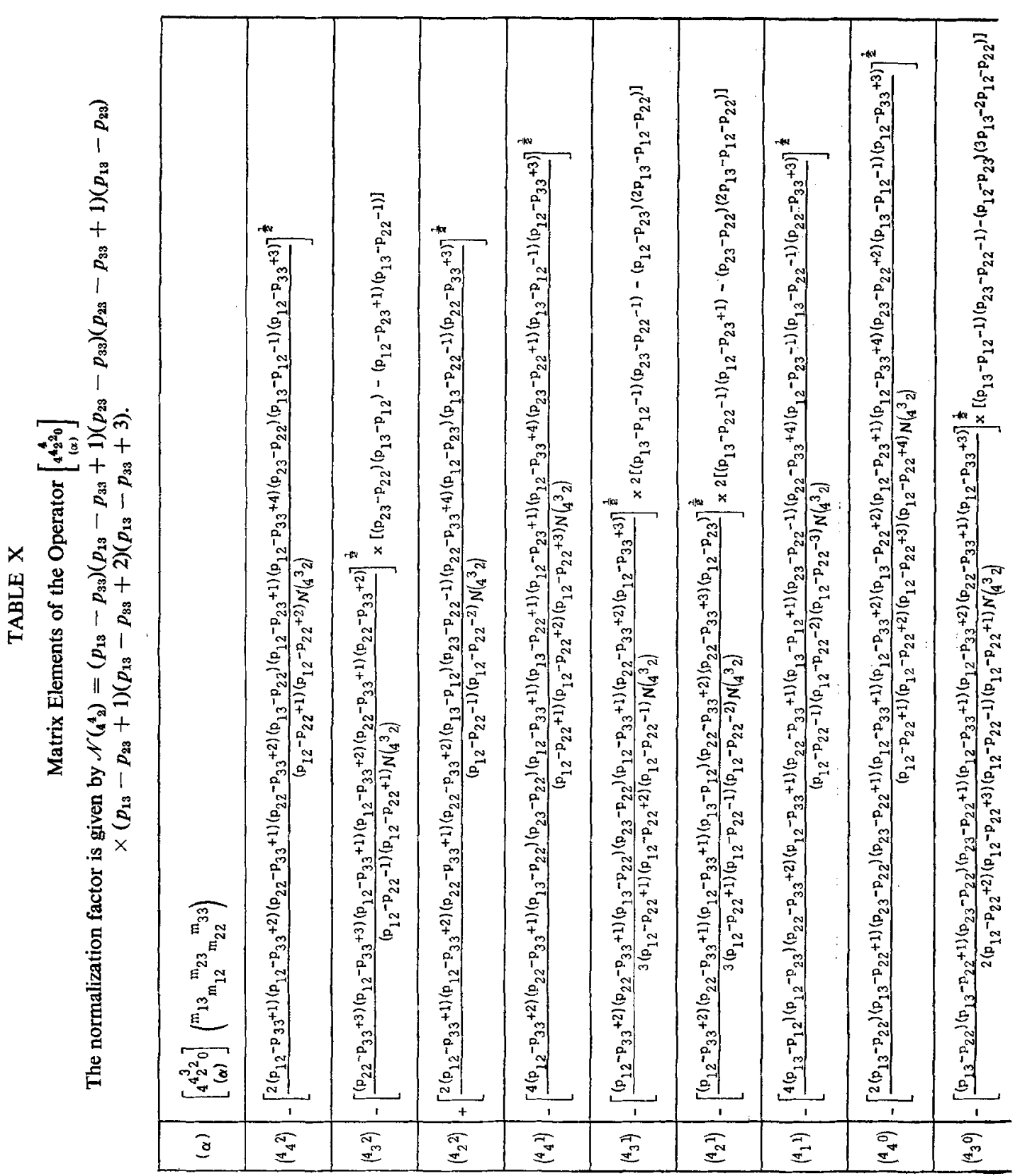




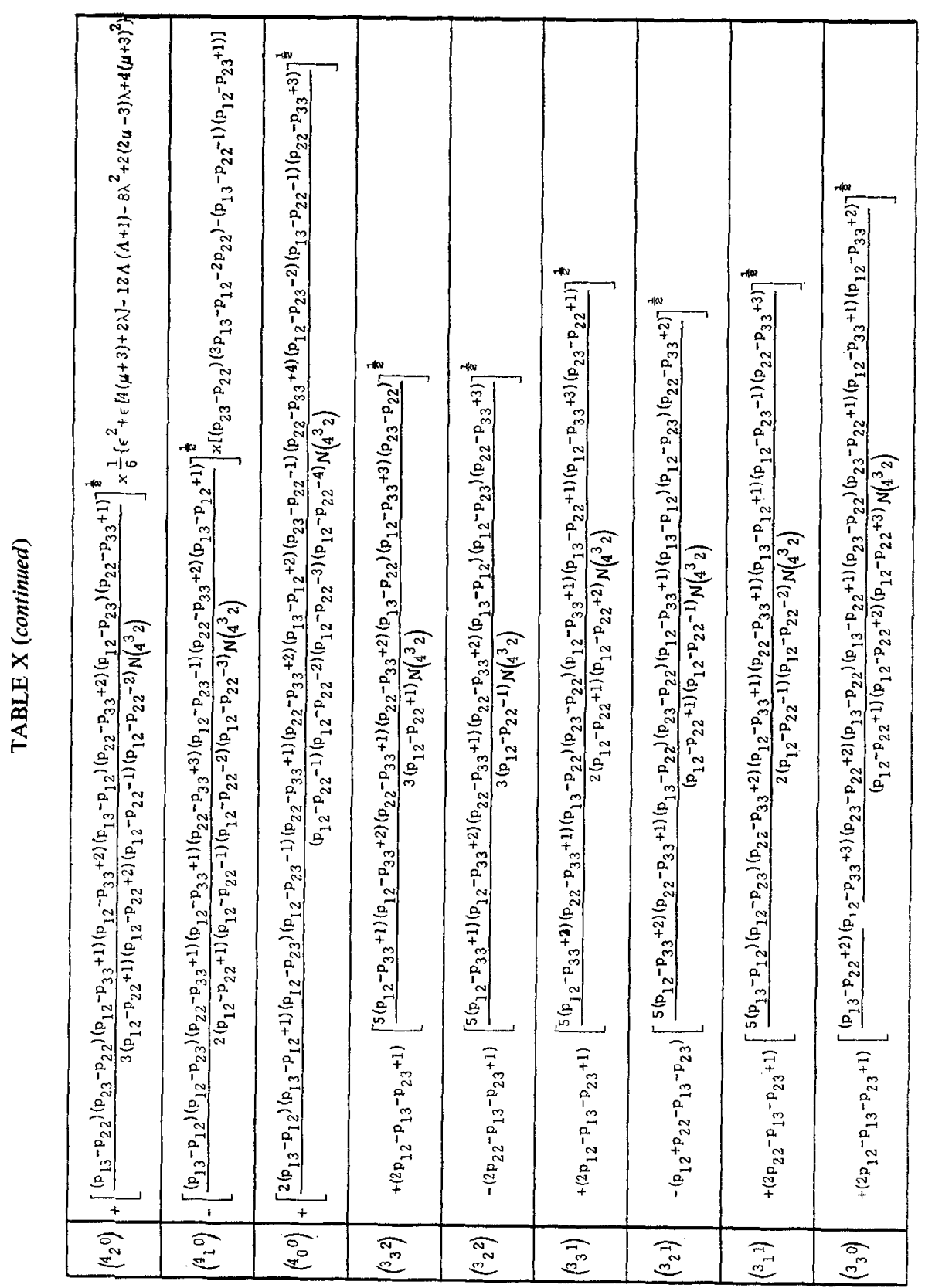




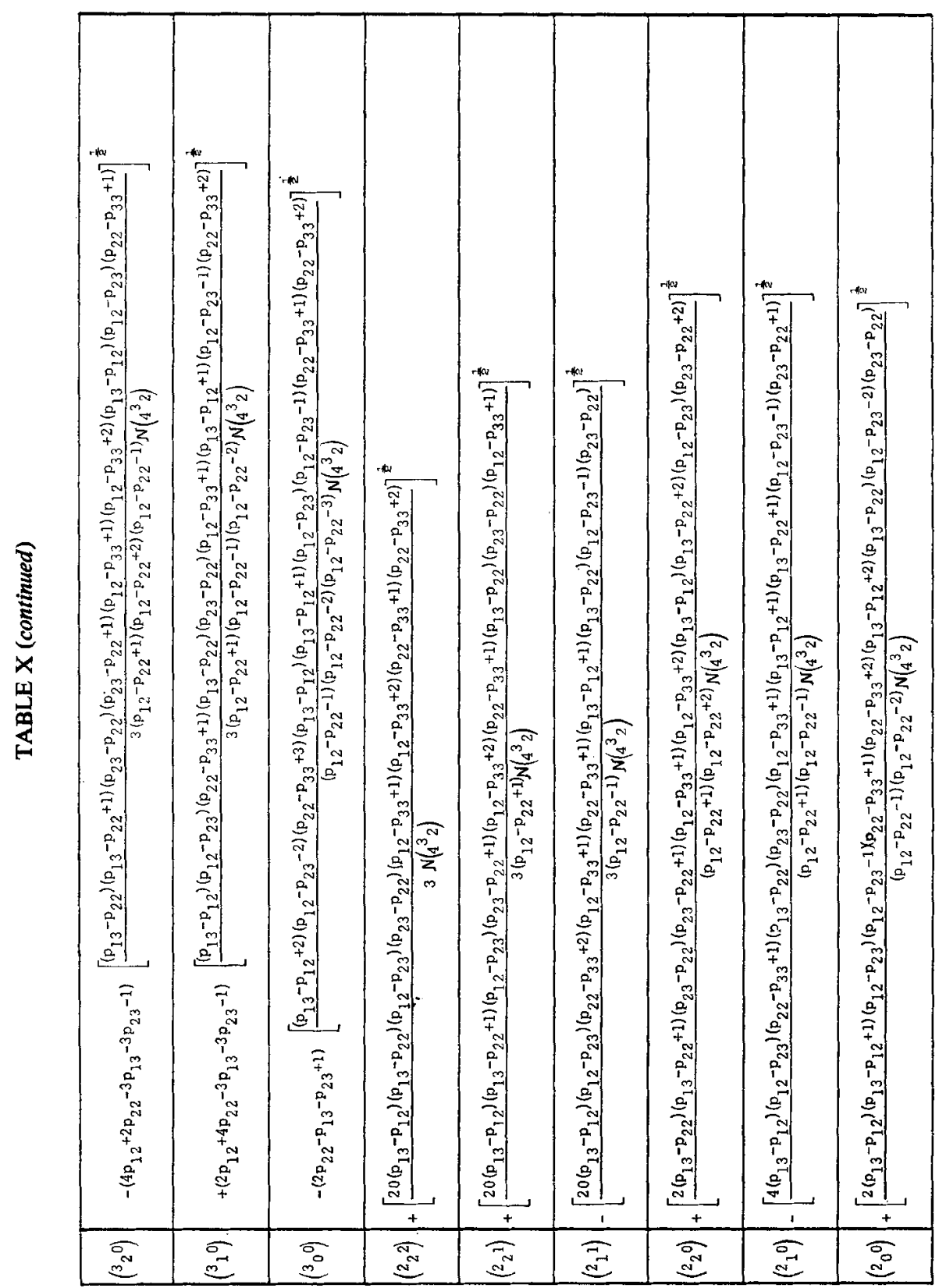


ON THE 27-PLET UNITARY SYMMETRY OPERATOR

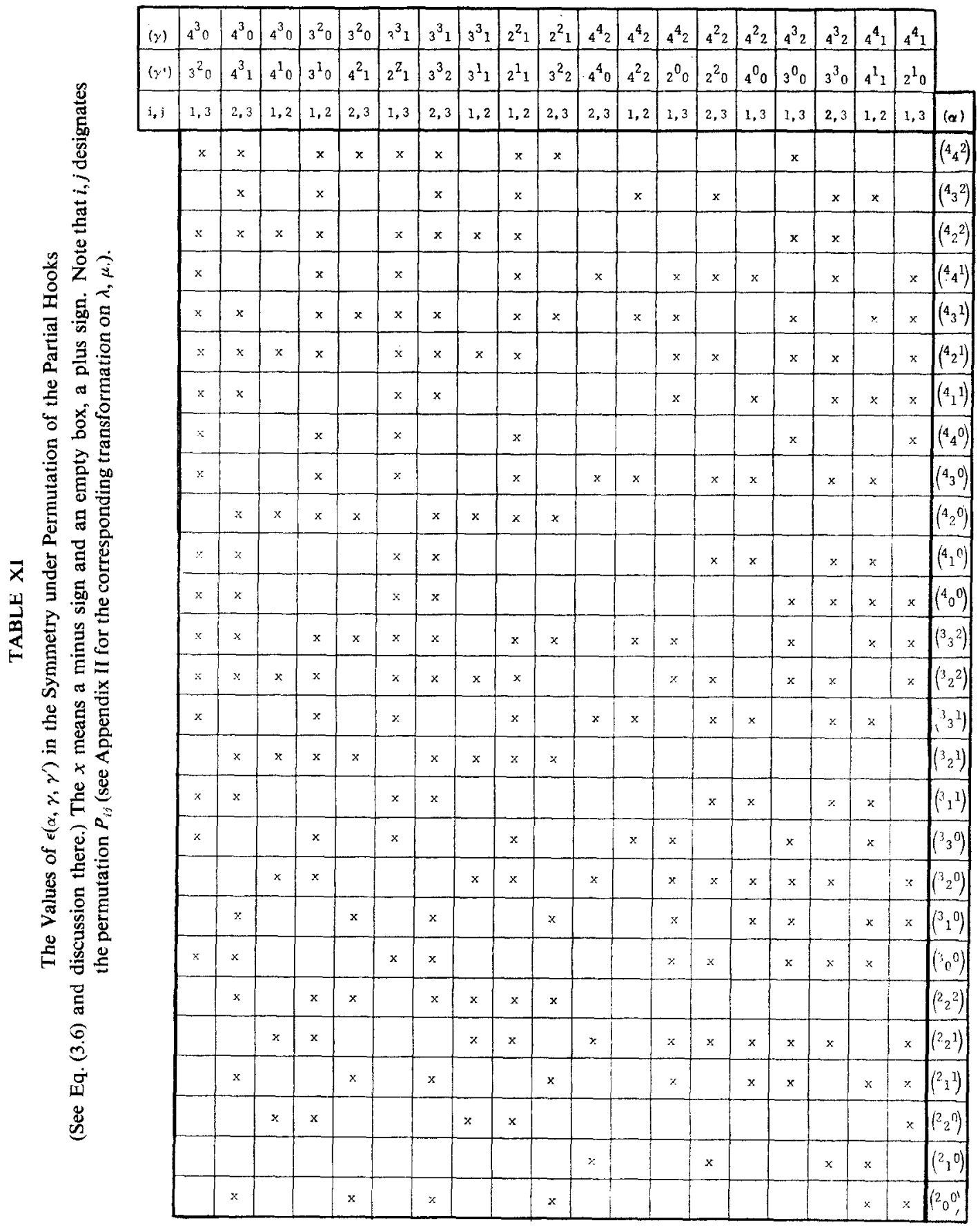




\section{APPENDIX I}

We give here a tabulation of the Racah coefficients $U(\ldots)$ discussed in Section $4, B$. The Racah coefficients are defined by a recoupling equation involving three $S U(3)$ irreps, in complete analogy with the $S U(2)$ Racah coefficients:

$$
\begin{aligned}
& \left.\sum_{(\alpha)_{12}}\left\langle\left\{\begin{array}{c}
{[m]_{12}} \\
(\alpha)_{12}
\end{array}\left|\left\langle\begin{array}{c}
(\gamma)_{12} \\
{[m]_{2}} \\
(\alpha)_{2}
\end{array}\right)\right| \begin{array}{c}
{[m]_{1}} \\
(\alpha)_{1}
\end{array}\right\rangle\left\langle\begin{array}{c}
{[m]} \\
(\alpha)
\end{array}\right)\right| \begin{array}{c}
(\gamma)_{12,3} \\
{[m]_{3}} \\
(\alpha)_{3}
\end{array}\right)\left|\begin{array}{c}
{[m]_{12}} \\
(\alpha)_{12}
\end{array}\right\rangle \\
& =\sum_{\substack{(\alpha)_{23} \\
(\gamma)_{23}(\gamma)_{1,23}}}\left\langle\begin{array}{c}
{[m]_{23}} \\
(\alpha)_{23}
\end{array}\left|\left\langle\begin{array}{c}
(\gamma)_{23} \\
{[m]_{3}} \\
(\alpha)_{3}
\end{array}\right)\right| \begin{array}{c}
{[m]_{2}} \\
(\alpha)_{2}
\end{array}\right\rangle\left\langle\begin{array}{c}
{[m]_{3}} \\
(\alpha)_{3}
\end{array}\left|\left\langle\begin{array}{c}
(\gamma)_{1,23} \\
{[m]_{23}} \\
(\alpha)_{23}
\end{array}\right)\right| \begin{array}{c}
{[m]_{1}} \\
(\alpha)_{1}
\end{array}\right\rangle \\
& \times U\left([m]_{1}[m]_{2}[m][m]_{3} ;[m]_{12}(\gamma)_{12}(\gamma)_{12,3} ;[m]_{23}(\gamma)_{23}(\gamma)_{1,23}\right),
\end{aligned}
$$

where we use the abbreviated notation $[m] \equiv\left(m_{13} m_{23} m_{33}\right)$ or $(\lambda \mu)$,

$$
(\alpha)=\left(\begin{array}{ll}
\alpha_{12} & \alpha_{22} \\
& \alpha_{11}
\end{array}\right) \quad \text { or } \quad\left(\epsilon \Lambda M_{\Lambda}\right) \quad \text { and } \quad(\gamma)=\left(\begin{array}{cc}
c & \gamma_{11} \\
\gamma_{12} & \gamma_{22}
\end{array}\right) .
$$

Some properties of the Racah coefficients are more evident when we express them in terms of Wigner coefficients. Using the orthogonality properties of the Wigner coefficients Eq. (I-1) becomes

$$
\begin{aligned}
& U\left([m]_{1}[m]_{2}[m][m]_{3} ;[m]_{12}(\gamma)_{12}(\gamma)_{12,3} ;[m]_{23}(\gamma)_{23}(\gamma)_{1,23}\right) \\
&\left.=\sum_{\substack{(\alpha)_{1}(\alpha)_{2}(\alpha)_{3} \\
(\alpha)_{12}(\alpha)_{23}}}\left\langle\begin{array}{c}
m]_{12} \\
(\alpha)_{12}
\end{array}\right| \begin{array}{c}
(\gamma)_{12} \\
{[m]_{2}} \\
(\alpha)_{2}
\end{array}\right\}\left|\begin{array}{c}
{[m]_{1}} \\
(\alpha)_{1}
\end{array}\right\rangle \\
& \times\left\langle\begin{array}{c}
m \\
(\alpha)
\end{array}\right) \\
&
\end{aligned}
$$

Similarly, from (I-1) we get

$$
\begin{gathered}
\sum_{(\alpha)_{12}(\alpha)_{2}}\left\langle\begin{array}{c}
{[m]} \\
(\alpha))_{3}
\end{array}\left|\left\langle\begin{array}{c}
(\gamma)_{12,3} \\
{[m]_{3}} \\
(\alpha)_{3}
\end{array}\right\rangle\right| \begin{array}{c}
{[m]_{12}} \\
(\alpha)_{12}
\end{array}\right\rangle\left\langle\begin{array}{c}
{[m]_{12}} \\
(\alpha)_{12}
\end{array}\left|\left\langle\begin{array}{c}
(\gamma)_{12} \\
{[m]_{2}} \\
(\alpha)_{2}
\end{array}\right\rangle\right| \begin{array}{c}
{[m]_{1}} \\
(\alpha)_{1}
\end{array}\right\rangle\left\langle\begin{array}{c}
{[m]_{23}} \\
(\alpha)_{23}
\end{array}\left|\left\langle\begin{array}{c}
(\gamma)_{23} \\
{[m]_{3}} \\
(\alpha)_{3}
\end{array}\right\rangle\right| \begin{array}{c}
{[m]_{2}} \\
(\alpha)_{2}
\end{array}\right\rangle \\
=\sum_{(\gamma)_{1,23}}\left\langle\begin{array}{c}
{[m]} \\
(\alpha)
\end{array}\right)\left\langle\begin{array}{c}
(\gamma)_{1,23} \\
{[m]_{23}} \\
(\alpha)_{23}
\end{array}\right)\left|\begin{array}{c}
{[m]_{1}} \\
(\alpha)_{1}
\end{array}\right\rangle U\left([m]_{1}[m]_{2}[m][m]_{3} ;(\gamma)_{12}(\gamma)_{12,3} ;(\gamma)_{23}(\gamma)_{1,23}\right)
\end{gathered}
$$

(Equation (2.25) in the text is a particular instance of I-3.) 
We use next Eq. (I-1) and (I-3) to obtain the Racah coefficient for the recoupling of $(\lambda \mu) \otimes(20) \otimes(02)$ into $(\lambda \vec{\mu})$. (To find all the possibilities for this recoupling it is helpful to use the graphical description of the Racah coefficient as a complete quadrilateral.)

The possibilities for $(\bar{\lambda} \bar{\mu})$ are of three types:

(1) $(\bar{\lambda} \bar{\mu})=(\lambda \mu)$;

(2) $(\bar{\lambda} \bar{\mu})=(\lambda+1, \mu+1),(\lambda+2, \mu-1),(\lambda-1, \mu+2),(\lambda-2, \mu+1)$, $(\lambda+1, \mu-2),(\lambda-1, \mu-1)$;

(3) $(\bar{\lambda} \bar{\mu})=(\lambda+2, \mu+2),(\lambda+3, \mu),(\lambda+4, \mu-2),(\lambda, \mu+3)$, $(\lambda-2, \mu+4),(\lambda-3, \mu+3),(\lambda-4, \mu+2),(\lambda+3, \mu-3)$, $(\lambda-3, \mu),(\lambda+2, \mu-4),(\lambda, \mu-3),(\lambda-2, \mu-2)$.

The value $(\bar{\lambda} \bar{\mu})=(\lambda \mu)$ can be obtained by all the six possibilities for $\left(\lambda_{12} \mu_{12}\right)$ [or $\left(\lambda_{23} \mu_{23}\right),(\gamma)_{1,23}$, then its tabulation is done by a $6 \times 6$ table (Table A-2). The possibilities for $(\bar{\lambda} \bar{\mu})$ of type (2) can be obtained by only three possibilities for $\left(\lambda_{12} \mu_{12}\right)$ [or $\left.\left(\lambda_{23} \mu_{23}\right),(\gamma)_{1,23}\right]$, then its tabulation involves six tables in the form of a $3 \times 3$ matrix. As a typical example, we give in Table A-1 the Racah coefficients for $(\bar{\lambda} \bar{\mu})=(\lambda+1, \mu+1)$. Racah coefficients for the remaining possibilities of type (2) can be obtained from this table and the symmetry under permutation of $U(3)$ partial hooks. The values $(\bar{\lambda} \bar{\mu})$ of type (3) can be obtained by only one possibility for $\left(\lambda_{12} \mu_{12}\right)$ [or $\left(\lambda_{23} \mu_{23}\right),(\gamma)_{1,23}$ ] and their Racah coefficients are unity.

\section{TABLE A-1}

$$
\begin{gathered}
\text { Racah Coefficients } U\left((\lambda \mu)(20)(\lambda+1, \mu+1)(02) ;\left(\lambda_{12} \mu_{12}\right)--;\left(\lambda_{23} \mu_{23}\right)-(\gamma)\right) \\
\text { (Here } \left.\pi=\pi(\lambda \mu)=\left(3 \lambda^{2}+4 \lambda \mu+3 \mu^{2}+15 \lambda+15 \mu\right) .\right)
\end{gathered}
$$

\begin{tabular}{|c|c|c|c|}
\hline & (11), $\left(2_{2}^{2}\right)$ & $(22),\left(3^{3} 2\right)$ & (22). $\left(4^{3} y\right)$ \\
\hline$(\lambda, \mu+1)$ & {$\left[\frac{\lambda(\mu+3)}{5(\lambda+2)(\mu+1)}\right]^{\frac{1}{2}}$} & $\langle\lambda-\mu+5\rangle\left[\frac{2 \lambda(\mu+3)}{5(\lambda+2)(\mu+1) \pi}\right]^{\frac{1}{2}}$ & {$\left[\frac{2(\lambda+3) \mu(\lambda+\mu+1)(\lambda+\mu+5)}{(\lambda+2)(\mu+1) \pi}\right]^{\frac{1}{2}}$} \\
\hline$(\lambda+2, \mu)$ & $-\left[\frac{2(\lambda+3)(\lambda+\mu+5)}{5(\lambda+2)(\lambda+\mu+3)}\right]^{\frac{1}{2}}$ & $(3 \lambda+2 \mu)\left[\frac{(\lambda+3)(\lambda+\mu+5)}{5(\lambda+2)(\lambda+\mu+3) \pi}\right]^{\frac{1}{2}}$ & {$\left[\frac{\lambda u(\mu+3)(\lambda+\mu+1)}{(\lambda+2)(\lambda+\mu+3) \pi}\right]^{\frac{1}{2}}$} \\
\hline$(\lambda+1, \mu-1)$ & {$\left[\frac{2 \mu(\lambda+\mu+1)}{5(\mu+1)(\lambda+\mu+3)}\right]^{\frac{2}{2}}$} & $(2 \lambda+3 \mu+15)\left[\frac{\mu(\lambda+\mu+1)}{5(\mu+1)(\lambda+\mu+3) \pi}\right]^{\frac{1}{2}}$ & $-\left[\frac{\lambda(\lambda+3)(\mu+3)(\lambda+\mu+5)}{(\mu+1)(\lambda+\mu+3) \pi}\right]^{\frac{1}{2}}$ \\
\hline
\end{tabular}



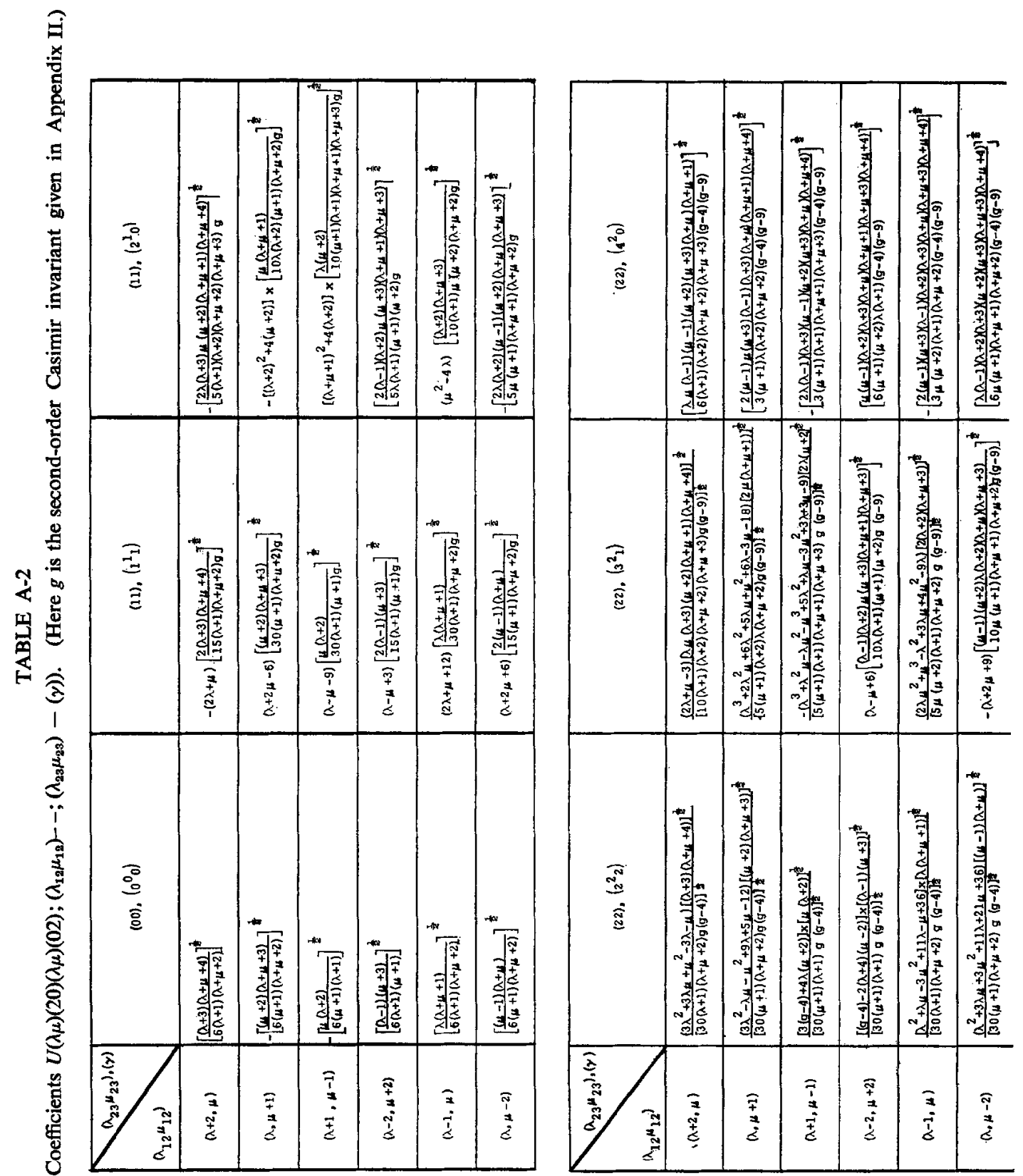

急 
Specializing (I-3) to the recoupling in question, we see that the three types of possibility for $(\bar{\lambda} \bar{\mu})$ and the different ways they can be built from $\left(\lambda_{12} \mu_{12}\right)$ [or $\left(\lambda_{23} \mu_{23}\right)$ $\left.(\gamma)_{1,23}\right]$ are intimately connected with the multiplicity of the weight diagram of

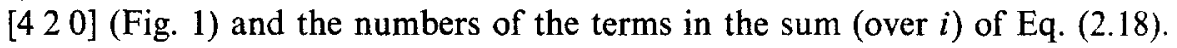

\section{APPENDIX II}

We present now a translation guide for the different notations used in the various fields to which our calculations are relevant. Given a $U(3)(S U(3))$ Gelfand pattern

$$
\left(\begin{array}{ccc}
m_{13} & m_{23} & m_{33} \\
& m_{12} & m_{22}
\end{array}\right)
$$

we call "weight" of the state whose labels are given by this pattern, the triplet of numbers

$$
\Delta_{3}=\left(\Delta_{13}, \Delta_{23}, \Delta_{33}\right)=\left(m_{11}, m_{12}+m_{22}-m_{11}, m_{13}+m_{23}+m_{33}-m_{12}-m_{22}\right) .
$$

The connections with the $S U(3)$ labels used in particle physics $\left(I, I_{z}, Y\right)$ and the Elliott variables $\left(\Lambda, M_{\Lambda}, \epsilon\right)$ used in nuclear physics are

$$
\begin{aligned}
I & =1 / 2\left(m_{12}-m_{22}\right)=\Lambda, \\
I_{z} & =1 / 2\left(2 m_{11}-m_{12}-m_{22}\right)-M_{\Lambda}, \\
Y & =1 / 3\left[3\left(m_{12}+m_{22}\right)-2\left(m_{13}+m_{23}+m_{33}\right)\right]=-\epsilon / 3 .
\end{aligned}
$$

The connection between the different variables used in this paper is

$$
\begin{aligned}
p_{i j} & =m_{i j}+j-i \text { (called a "partial hook"), } \\
\lambda & =p_{13}-p_{23}-1=m_{13}-m_{23}, \\
\mu & =p_{23}-p_{33}-1=m_{23}-m_{33} .
\end{aligned}
$$

The converse relations are then:

$$
\begin{aligned}
p & =p_{12}-p_{23}=m_{12}-m_{23} \\
q & =p_{22}-p_{23}=m_{22}-m_{33} \\
\epsilon & =(2 \lambda+\mu-3 p-3 q)=2\left(p_{13}+p_{23}+p_{33}\right)-3\left(p_{12}+p_{22}\right)-3 \\
A & =1 / 2(\mu+p-q)=1 / 2\left(p_{12}-p_{22}-1\right)=1 / 2\left(m_{12}-m_{22}\right) .
\end{aligned}
$$


The $S U(3)$ Casimir invariants are denoted:

$$
\begin{aligned}
g & =\text { Second-order Casimir invariant }=\lambda^{2}+\mu^{2}+\lambda \mu+3(\lambda+\mu) \\
& =\left(p_{13}^{2}+p_{23}^{2}+p_{33}^{2}-p_{13} p_{23}-p_{13} p_{33}-p_{23} p_{33}-3\right)=9 I_{2} \\
\Gamma & =\text { Third-order Casimir invariant }=(\lambda-\mu)(2 \lambda+\mu+3)(\lambda+2 \mu+3) \\
& =-\left(p_{13}-2 p_{23}+p_{33}\right)\left(-2 p_{13}+p_{23}+p_{33}\right)\left(p_{13}+p_{23}-2 p_{33}\right) \equiv 162 I_{3} .
\end{aligned}
$$

The permutations of the partial hooks, in Elliott's notation become

$$
\begin{aligned}
& p_{13} \leftrightarrow p_{23} \Rightarrow(\lambda \rightarrow-\lambda-2, \mu \rightarrow \lambda+\mu+1), \\
& p_{13} \leftrightarrow p_{33} \Rightarrow(-\mu-2,-\lambda-2), \\
& p_{23} \leftrightarrow p_{33} \Rightarrow(\lambda+\mu+1,-\mu-2), \\
& p_{12} \leftrightarrow p_{22} \Rightarrow(\Lambda \rightarrow-\Lambda-1) .
\end{aligned}
$$

The $U(2)$ Wigner operators are directly related to the $S U(2)$ Clebsch Gordan Coefficients through

$$
\begin{aligned}
& \left\langle m_{12}+\mu_{11} m_{11}+M_{11} m_{22}+M_{12}+M_{22}-\mu_{11}\right|\left\langle M_{12} M_{11}^{\mu_{22}}|| \begin{array}{ll}
m_{12} & m_{22} \\
M_{11}
\end{array} m_{11}\right\rangle \\
& =\left\langle J M^{\frac{1}{2}}\left(M_{12}-M_{22}\right) \frac{1}{2}\left(2 M_{11}-M_{12}-M_{22}\right)\right| J+\frac{1}{2}\left(2 \mu_{11}-M_{12}-M_{22}\right) M \\
& \left.+\frac{1}{2}\left(2 M_{11}-M_{12}-M_{22}\right)\right\rangle \text {, }
\end{aligned}
$$

where

$$
\begin{aligned}
J & =\frac{1}{2}\left(m_{12}-m_{22}\right)=\frac{1}{2}\left(p_{12}-p_{22}-1\right)=\Lambda \\
M & =\frac{1}{2}\left(2 m_{11}-m_{12}-m_{22}\right)=\frac{1}{2}\left(2 p_{11}-p_{12}-p_{22}+1\right)=M_{\Lambda} .
\end{aligned}
$$

\section{REFERENCES}

1. G. E. Baird ANd L. C. Biedenharn, J. Math. Phys. 4 (1963), 1449; 5 (1964), 1730; 6 (1965), 1847.

2. E. P. Wigner, Bull. Amer. Math. Soc. 74 (1968), 793.

3. L. C. Biedenharn, A. Giovannini, And J. D. Louck, J. Math. Phys. 8 (1967), 691.

4. J. J. De Swart, Rev. Mod. Phys. 35 (1963), 916.

5. L. C. Biedenharn and J. D. Louck, Commun. Math. Phys. 8 (1968), 89.

6. K. T. HeChT, Nucl. Phys. 62 (1965), 1.

7. J. D. Louck, to be published. 
8. H. WeYL, "Classical Groups," Princeton University Press, Princeton, N. J., 1946.

9. S. J. Alisauskas and A. P. JucYs, J. Math. Phys. 8 (1967), 2250.

10. R. E. Cutkosky, Ann. Phys. (New York) 23 (1963), 415; P. Carruthers, "Introduction to Unitary Symmetry," p. 131 ff., Wiley-Interscience, New York, 1966.

11. K. C. Wali and R. Warnock, Phys. Rev. B 135 (1964), 1358.

12. E. Golowich, Phys. Rev. B 134 (1965), 1297.

13. S. OKuBo, Phys. Lett. 4 (1963), 14.

14. D. Bailin, Nuovo Cimento 38 (1965), 1342; K. C. GuPta And R. Majumdar, Nuovo Cimento 37 (1965), 1801.

15. R. Dashen and M. Weinstein, I.A.S. Preprint, June 1969.

16. F. Gursey, T. D. Lee, And M. Nauenberg, Phys. Rev. B 135 (1964), 467. See also: Dalitz, Ref. (17) and L. C. BIEDENHARN, CERN Lectures, CERN 65-41 (1965), unpublished.

17. R. H. Dalitz, in "Lectures on High Energy Physics, Les Houches," (C. DeWitt and M. Jacob, Eds.), Gordon and Breach, New York, 1965.

18. J. D. Vergados, Nucl. Phys. A 111 (1968), 681.

19. S. C. PANG AND K. T. Hecht, J. Math. Phys. 10 (1969), 1571. 